\title{
Charged Lepton Flavour Violation in Supersymmetric Low-Scale Seesaw Models
}

\author{
Amon Ilakovac $^{a}$, Apostolos Pilaftsis ${ }^{b}$ and Luka Popov ${ }^{a}$ \\ ${ }^{a}$ University of Zagreb, Department of Physics, Bijenička cesta 32, P.O. Box 331, Zagreb, Croatia \\ ${ }^{b}$ Consortium for Fundamental Physics, School of Physics and Astronomy, \\ University of Manchester, Manchester M13 9PL, United Kingdom
}

\begin{abstract}
We study charged lepton flavour violation in low-scale seesaw models of minimal supergravity, which realize large neutrino Yukawa couplings thanks to approximate lepton-number symmetries. There are two dominant sources of lepton flavour violation in such models. The first source originates from the usual soft supersymmetry-breaking sector, whilst the second one is entirely supersymmetric and comes from the supersymmetric neutrino Yukawa sector. Within the framework of minimal supergravity, we consider both sources of lepton flavour violation, soft and supersymmetric, and calculate a number of possible lepton-flavour-violating transitions, such as the photonic decays of muons and taus, $\mu \rightarrow e \gamma, \tau \rightarrow e \gamma$ and $\tau \rightarrow \mu \gamma$, their neutrinoless three-body decays, $\mu \rightarrow e e e$, $\tau \rightarrow e e e, \tau \rightarrow \mu \mu \mu, \tau \rightarrow e e \mu$ and $\tau \rightarrow e \mu \mu$, and the coherent $\mu \rightarrow e$ conversion in nuclei. After taking into account the exclusion bounds placed by present experiments of lepton flavour violation, we derive combined theoretical limits on the universal heavy Majorana mass scale $m_{N}$ and the light-to-heavy neutrino mixings. Supersymmetric low-scale seesaw models offer distinct correlated predictions for lepton-flavour-violating signatures, which might be discovered in current and projected experiments, such as MEG, COMET/PRISM, Mu2e, super-BELLE and LHCb.

PACS numbers: $11.30 \mathrm{Hv}, 12.60 \mathrm{Jv}, 14.60 . \mathrm{Pq}$
\end{abstract}

\section{INTRODUCTION}

Neutrino oscillation experiments [1-4] have provided undisputed evidence of Lepton Flavour Violation (LFV) in the neutrino sector, pointing towards physics beyond the Standard Model (SM). Recent reactor neutrino oscillation experiments [5] have shown that the angle $\theta_{13}$ of the Pontecorvo-Maki-Nakagawa-Sakata (PMNS) mixing matrix [6] is nonzero, thus hinting at a non-trivial neutrino-flavour structure and possibly at CP violation. Nevertheless, in spite of intense experimental searches [7-11], no evidence of LFV has been found yet in the charged lepton sector of the SM, implying conservation of the individual lepton numbers associated with the electron $e$, the muon $\mu$ and the tau $\tau$. All past and current experiments were only able to report upper limits on observables of Charged Lepton Flavour Violation (CLFV).

Recently, the MEG collaboration [7] has announced an improved upper limit on the branching ratio of the CLFV decay $\mu \rightarrow e \gamma$, with $B(\mu \rightarrow e \gamma)<2.4 \times 10^{-12}$ at the $90 \%$ confidence level (CL). As also shown in Table 【, future experiments searching for the CLFV processes, $\mu \rightarrow e \gamma, \mu \rightarrow e e e$, coherent $\mu \rightarrow e$ conversion in nuclei, $\tau \rightarrow e \gamma / \mu \gamma$, $\tau \rightarrow 3$ leptons and $\tau \rightarrow$ lepton+light meson, are expected to reach branching-ratio sensitivities to the level of $10^{-13}[12$ $\left(10^{-14}[13]\right), 10^{-16}[14]\left(10^{-17}[13]\right), 10^{-17}[15,16]\left(10^{-18}[13,17]\right), 10^{-9}[18,19], 10^{-10}[18]$ and $10^{-10}$ [18], respectively. The values in parentheses indicate the sensitivities that are expected to be achieved by the new generation CLFV experiments in the next decade. Most interestingly, the projected sensitivity for $\mu \rightarrow$ eee and $\mu \rightarrow e$ conversion in nuclei is expected to increase by five and six orders of magnitude, respectively. Given that CLFV is forbidden in the SM, its observation would constitute a clear signature for New Physics, thus rendering this field of investigations even more exciting.

Although forbidden in the SM, CLFV is a generic feature for most of its extensions. One such well-motivated extension is the so-called Minimal Supersymmetric Standard Model (MSSM) 20], where supersymmetry (SUSY) is softly broken at the 1-10 TeV scale for phenomenological reasons. The MSSM provides a quantum-mechanically stable solution to the gauge hierarchy problem and predicts rather accurate unification of the SM gauge couplings close to the Grand Unified Theory (GUT) scale. If R-parity is conserved, the lightest supersymmetric particle (LSP) is stable and, if neutral, such as the neutralino, it could play the role of the Dark Matter (DM) in the Universe. Finally, the MSSM typically predicts a SM-like Higgs boson lighter than $135 \mathrm{GeV}$, in agreement with the recent observations for a 125-GeV Higgs boson, made by the ATLAS 21] and CMS [22] collaborations. 


\begin{tabular}{|c|c|c|}
\hline No. Observable & Upper Limit & Future Sensitivity \\
\hline 1. $B(\mu \rightarrow e \gamma)$ & $2.4 \times 10^{-12}[7]$ & $1-2 \times 10^{-13}[12], 10^{-14}[13]$ \\
\hline 2. $B(\mu \rightarrow e e e)$ & $10^{-12}[8]$ & $10^{-16}[14], 10^{-17}[13]$ \\
\hline 3. $R_{\mu e}^{\mathrm{Ti}}$ & $4.3 \times 10^{-12}[9]$ & $3-7 \times 10^{-17}[15,16], 10^{-18}[13,17]$ \\
\hline 4. $R_{\mu e}^{\mathrm{Au}}$ & $7 \times 10^{-13}[10]$ & $3-7 \times 10^{-17}[15,16], 10^{-18}[13,17]$ \\
\hline 5. $B(\tau \rightarrow e \gamma)$ & $3.3 \times 10^{-8}[11]$ & $1-2 \times 10^{-9}[18,19]$ \\
\hline 6. $B(\tau \rightarrow \mu \gamma)$ & $4.4 \times 10^{-8}[11]$ & $2 \times 10^{-9}[18,19]$ \\
\hline 7. $B(\tau \rightarrow e e e)$ & $2.7 \times 10^{-8}[11]$ & $2 \times 10^{-10}[18,19]$ \\
\hline 8. $B(\tau \rightarrow e \mu \mu)$ & $2.7 \times 10^{-8}[11]$ & $10^{-10}[18]$ \\
\hline 9. $B(\tau \rightarrow \mu \mu \mu)$ & $2.1 \times 10^{-8}[11]$ & $2 \times 10^{-10}[18,19]$ \\
\hline 10. $B(\tau \rightarrow \mu e e)$ & $1.8 \times 10^{-8}[11]$ & $10^{-10}[18]$ \\
\hline
\end{tabular}

TABLE I: Current upper limits and future sensitivities of CLFV observables under study.

In the MSSM with R-parity conservation, the lepton number is preserved and all left-handed light neutrinos $\nu_{e, \mu, \tau}$ remain massless, exactly as in the SM. To account for the observed light neutrino masses and mixings, while maintaining R-parity, the field content of the MSSM needs to be extended. An interesting extension to the MSSM is provided by the so-called seesaw mechanism. There are three realizations of the seesaw mechanism: the seesaw type I [23], the seesaw type II 24] and the seesaw type III [25]. The three scenarios differ by the nature of their seesaw messengers that are needed to explain the small neutrino masses. In this study, we will adopt a low-scale variant of the seesaw type-I realization, whose messengers are three singlet heavy neutrinos $N_{1,2,3}$.

In the usual seesaw type-I mechanism, the heavy singlet neutrinos must assume masses of order $\sim 10^{12-14} \mathrm{GeV}$, for electroweak-scale Dirac neutrino masses, in order to account for the observed light neutrino mass spectrum. The mixing between light and heavy neutrinos is of the order $\xi_{\nu N} \sim \sqrt{m_{\nu} / m_{N}} \sim 10^{-12}$, for light-neutrino masses $m_{\nu} \sim 10^{-1} \mathrm{eV}$. As a consequence, heavy neutrinos decouple from low-energy processes of CLFV in the SM with right-handed neutrinos, giving rise to extremely suppressed and unobservable rates. In the MSSM with right-handed neutrinos, however, the singlet heavy neutrinos do not fully decouple. They impact the low-energy sector, through renormalization group (RG) effects that induce sizeable LFV in the slepton sector for a SUSY mass scale $M_{\mathrm{SUSY}} \sim 1-10 \mathrm{TeV}$.

A potentially interesting alternative to the ordinary seesaw mechanism may arise from the presence of approximate lepton-number symmetries [26 29] in the theory. The smallness of the light neutrino masses is a consequence of these approximate leptonic symmetries which are radiatively stable [29, 30], whilst the heavy neutrino mass scale could be as low as $100 \mathrm{GeV}$. Unlike in the usual seesaw scenario, the light-to-heavy neutrino mixings $\xi_{\nu N}$ are not correlated to the light neutrino masses $m_{\nu}$. Instead, $\xi_{\nu N}$ are free parameters, constrained by experimental limits on deviations of the $W^{ \pm}$- and $Z$-boson couplings to leptons with respect to their SM values [31, 32]. Approximate lepton-number symmetries do not restrict the size of LFV, and so potentially large phenomena of CLFV may be predicted. This feature is quite generic both in the SM [33] and in the MSSM [34, 35] augmented with low-scale right-handed neutrinos. It is this new source for LFV in the MSSM that we wish to study in detail here, in addition to the one resulting from the frequently considered soft SUSY breaking sector [36 39].

In this article, we denote for brevity the SM and the MSSM extended by low-scale right-handed neutrinos and approximate lepton-number symmetries by $\nu_{R} \mathrm{SM}$ and $\nu_{R} \mathrm{MSSM}$, respectively. Our study in this paper focuses on the $\nu_{R}$ MSSM with constrained boundary conditions at the gauge-coupling unification scale, within the framework of minimal supergravity (mSUGRA). However, the results presented here are applicable to more general soft SUSYbreaking scenarios.

The $\nu_{R}$ MSSM has some interesting features with respect to the MSSM. In particular, the heavy singlet sneutrinos may emerge as new viable candidates of Cold Dark Matter (CDM) [40]. In addition, the mechanism of low-scale resonant leptogenesis [30, 41] could provide a possible explanation for the observed Baryon Asymmetry in the Universe (BAU), as the parameter space for successful electroweak baryogenesis gets squeezed by the current LHC data [42].

Given the multitude of quantum states mediating LFV in the $\nu_{R} \mathrm{MSSM}$, the predicted values for observables of CLFV in this model turn out to be generically larger than the corresponding ones in the $\nu_{R} \mathrm{SM}$, except possibly for $B\left(l \rightarrow l^{\prime} \gamma\right)$ [34, 35], where $l, l^{\prime}=e, \mu, \tau$. The origin of suppression for the latter branching ratios may partially be attributed to the SUSY no-go theorem due to Ferrara and Remiddi [43], which states that the magnetic dipole moment operator necessarily violates SUSY and it has therefore to vanish in the supersymmetric limit of the theory.

The goal of this paper is to improve upon earlier calculations of CLFV in supersymmetric low-scale seesaw models which are either approximate, or incomplete. Specifically, in 34] a low-scale aligned neutrino and sneutrino spectrum 
was assumed to the leading order in the heavy neutrino mass, whilst in [44, 45] only a subset of loop effects mediated by the photon $\gamma$, the $Z$ boson, the neutral Higgs scalars and box graphs was considered. Instead, we present here detailed analytical expressions for CLFV observables, induced by $\gamma$ - and $Z$-boson formfactors, as well as the complete set of box contributions involving heavy neutrinos, sleptons, sneutrinos, charginos, neutralinos and charged Higgs bosons. We also derive the complete set of kinematic formfactors that contributes to the three-body LFV decays of the muon and tau, such as $\mu \rightarrow e e e, \tau \rightarrow e e e, \tau \rightarrow \mu \mu \mu, \tau \rightarrow e e \mu$ and $\tau \rightarrow e \mu \mu$. In our numerical analysis, we consider benchmark scenarios of the $\nu_{R}$ MSSM, which are in agreement with the existing LHC data from the recent discovery of a SM-like Higgs boson [21, 22] and the non-observation of squarks and gluinos [46].

The paper is organized as follows. In Section II we describe the relevant leptonic sector of the $\nu_{R}$ MSSM and introduce two baseline scenarios based on approximate lepton-number symmetries. Section III contains details of the calculation of the LFV amplitudes and branching ratios of CLFV decays of the tau and muon, where the complete set of chiral structures of the amplitudes contributing to the CLFV processes $l \rightarrow l^{\prime} \gamma, l \rightarrow l^{\prime} l_{1} \bar{l}_{2}$ and $\mu \rightarrow e$ conversion in nuclei are derived. Section IV presents numerical estimates for the aforementioned processes of CLFV, within the two baseline mSUGRA scenarios introduced in Section III. Moreover, we discuss correlated predictions of the CLFV observables with $B\left(l \rightarrow l^{\prime} \gamma\right)$ and other kinematic parameters. Section $\mathrm{V}$ summarizes the results of our analysis and presents our conclusions. All technical details have been relegated to the appendices. In Appendix $\mathrm{A}$, we give all relevant interaction vertices in the $\nu_{R}$ MSSM. Appendix B defines the one-loop functions that appear in our analytic calculations. In terms of these loop functions, Appendix Cdescribes the analytic results for all the one-loop formfactors that occur in the CLFV transition amplitudes.

\section{LOW-SCALE SEESAW MODELS AND LEPTON FLAVOUR VIOLATION}

In this section we describe the leptonic sector of the MSSM extended by low-scale right-handed neutrinos, which we call the $\nu_{R}$ MSSM for brevity. In addition, we introduce the neutrino Yukawa structure of two baseline scenarios based on approximate lepton-number symmetries and universal Majorana masses at the GUT scale. We will use these two scenarios to present generic predictions of CLFV within the framework of mSUGRA.

The leptonic superpotential part of the $\nu_{R}$ MSSM reads:

$$
W_{\text {lepton }}=\widehat{E}^{C} \mathbf{h}_{e} \widehat{H}_{d} \widehat{L}+\widehat{N}^{C} \mathbf{h}_{\nu} \widehat{L} \widehat{H}_{u}+\frac{1}{2} \widehat{N}^{C} \mathbf{m}_{M} \widehat{N}^{C},
$$

where $\widehat{H}_{u, d}, \widehat{L}, \widehat{E}$ and $\widehat{N}^{C}$ denote the two Higgs-doublet superfields, the three left- and right-handed charged-lepton superfields and the three right-handed neutrino superfields, respectively. The Yukawa couplings $\mathbf{h}_{e, \nu}$ and the Majorana mass parameters $\mathbf{m}_{M}$ form $3 \times 3$ complex matrices. Here, the Majorana mass matrix $\mathbf{m}_{M}$ is taken to be $\mathrm{SO}(3)$ symmetric at the GUT scale, i.e. $\mathbf{m}_{M}=m_{N} \mathbf{1}_{3}$.

In this study, we consider low-scale seesaw models [26 29], where the smallness of the light neutrino masses is protected by natural, quantum-mechanically stable cancellations due to the presence of approximate leptonic symmetries [29], whilst the Majorana mass scale $m_{N}$ can be as low as $100 \mathrm{GeV}$. In these models, the neutrino induced LFV transitions from a charged lepton $l=\mu, \tau$ to another charged lepton $l^{\prime} \neq l$ are functions of the ratios [33, 47, 48]

$$
\boldsymbol{\Omega}_{l^{\prime} l}=\frac{v_{u}^{2}}{2 m_{N}^{2}}\left(\mathbf{h}_{\nu}^{\dagger} \mathbf{h}_{\nu}\right)_{l^{\prime} l}=\sum_{i=1}^{3} B_{l^{\prime} N_{i}} B_{l N_{i}}
$$

and are not constrained by the usual seesaw factor $m_{\nu} / m_{N}$, where $v_{u} / \sqrt{2} \equiv\left\langle H_{u}\right\rangle$ is the vacuum expectation value (VEV) of the Higgs doublet $H_{u}$, with $\tan \beta \equiv\left\langle H_{u}\right\rangle /\left\langle H_{d}\right\rangle$. Moreover, the mixing matrix $B_{l N_{i}}$ that occurs in the interaction of the $W^{ \pm}$bosons with the charged leptons $l=e, \mu, \tau$ and the three heavy neutrinos $N_{1,2,3}$ is defined in Appendix $\mathrm{A}$. Note that the LFV parameters $\Omega_{l^{\prime} l}$ do not directly depend on the RG evolution of the soft SUSY-breaking parameters, except through the VEV $v_{u}$ at the minimum of the Higgs potential.

In the weak basis $\left\{\left(\nu_{e, \mu, \tau L}\right)^{C}, \nu_{1,2,3 R}\right\}$, the neutrino mass matrix in the $\nu_{R}$ MSSM takes on the standard seesaw type-I form [23]:

$$
\mathbf{M}_{\nu}=\left(\begin{array}{cc}
0 & \mathbf{m}_{D} \\
\mathbf{m}_{D}^{T} & \mathbf{m}_{M}^{*}
\end{array}\right)
$$

where $\mathbf{m}_{D}=\sqrt{2} M_{W} \sin \beta g_{w}^{-1} \mathbf{h}_{\nu}^{\dagger}$ and $\mathbf{m}_{M}$ are the Dirac- and Majorana-neutrino mass matrices, respectively. In this paper, we consider two baseline scenarios of neutrino Yukawa couplings. The first one realizes a U(1) leptonic 
symmetry [30] and is given by

$$
\mathbf{h}_{\nu}=\left(\begin{array}{lll}
0 & 0 & 0 \\
a e^{-\frac{i \pi}{4}} & b e^{-\frac{i \pi}{4}} & c e^{-\frac{i \pi}{4}} \\
a e^{\frac{i \pi}{4}} & b e^{\frac{i \pi}{4}} & c e^{\frac{i \pi}{4}}
\end{array}\right) .
$$

In the second scenario, the structure of the neutrino Yukawa matrix $\mathbf{h}_{\nu}$ is motivated by the discrete symmetry group $A_{4}$ and has the following form [49]:

$$
\mathbf{h}_{\nu}=\left(\begin{array}{lll}
a & b & c \\
a e^{-\frac{2 \pi i}{3}} & b e^{-\frac{2 \pi i}{3}} & c e^{-\frac{2 \pi i}{3}} \\
a e^{\frac{2 \pi i}{3}} & b e^{\frac{2 \pi i}{3}} & c e^{\frac{2 \pi i}{3}}
\end{array}\right)
$$

In (2.4) and (2.5), the Yukawa parameters $a, b$ and $c$ are assumed to be real. If the above leptonic (discrete or continuous) symmetries are not broken, the light neutrinos are massless. The neutrino masses are obtained by adding small terms that break the symmetry of the Yukawa matrix. It is essential to remark here that the predictions for CLFV are independent of the flavour structure of these small symmetry-breaking terms in our low-scale seesaw models under study. These terms are needed to fit the low-energy neutrino data. For this reason, we do not discuss here particular symmetry breaking patterns of the above two baseline Yukawa scenarios given in (2.4) and (2.5).

The second source of LFV in the models under consideration originates from scalar-neutrino (sneutrino) interactions, namely the supersymmetric partners of the left-handed and right-handed neutrinos. Specifically, the sneutrino mass Lagrangian in flavour and mass bases is given by

$$
\mathcal{L}^{\tilde{\nu}}=\left(\tilde{\nu}_{L}^{\dagger}, \tilde{\nu}_{R}^{C \dagger}, \tilde{\nu}_{L}^{T}, \tilde{\nu}_{R}^{C T}\right) \mathbf{M}_{\tilde{\nu}}^{2}\left(\begin{array}{c}
\tilde{\nu}_{L} \\
\tilde{\nu}_{R}^{C} \\
\tilde{\nu}_{L}^{*} \\
\tilde{\nu}_{R}^{C *}
\end{array}\right)=\tilde{N}^{\dagger} \mathcal{U}^{\tilde{\nu} \dagger} \mathbf{M}_{\tilde{\nu}}^{2} \mathcal{U}^{\tilde{\nu}} \tilde{N}=\tilde{N}^{\dagger} \widehat{\mathbf{M}}_{\tilde{\nu}}^{2} \tilde{N}
$$

where $\mathbf{M}_{\tilde{\nu}}^{2}$ is a $12 \times 12$ Hermitian mass matrix in the flavour basis and $\widehat{\mathbf{M}}_{\tilde{\nu}}^{2}$ is the corresponding diagonal mass matrix in the mass basis. More explicitly, in the flavour basis $\left\{\tilde{\nu}_{e, \mu, \tau L}, \tilde{\nu}_{1,2,3 R}^{C}, \tilde{\nu}_{e, \mu, \tau L}^{*}, \tilde{\nu}_{1,2,3 R}^{C *}\right\}$, the sneutrino mass matrix $\mathbf{M}_{\tilde{\nu}}^{2}$ may be cast into the form:

$$
\mathbf{M}_{\tilde{\nu}}^{2}=\left(\begin{array}{cccc}
\mathbf{H}_{1} & \mathbf{N} & \mathbf{0} & \mathbf{M} \\
\mathbf{N}^{\dagger} & \mathbf{H}_{2}^{T} & \mathbf{M}^{T} & \mathbf{0} \\
\mathbf{0} & \mathbf{M}^{*} & \mathbf{H}_{1}^{T} & \mathbf{N}^{*} \\
\mathbf{M}^{\dagger} & \mathbf{0} & \mathbf{N}^{T} & \mathbf{H}_{2}
\end{array}\right)
$$

where the block entries are the following $3 \times 3$ matrices

$$
\begin{aligned}
\mathbf{H}_{1} & =\mathbf{m}_{\tilde{L}}^{2}+\mathbf{m}_{D} \mathbf{m}_{D}^{\dagger}+\frac{1}{2} M_{Z}^{2} \cos 2 \beta \\
\mathbf{H}_{2} & =\mathbf{m}_{\tilde{\nu}}^{2}+\mathbf{m}_{D}^{\dagger} \mathbf{m}_{D}+\mathbf{m}_{M} \mathbf{m}_{M}^{\dagger} \\
\mathbf{M} & =\mathbf{m}_{D}\left(\mathbf{A}_{\nu}-\mu \cot \beta\right) \\
\mathbf{N} & =\mathbf{m}_{D} \mathbf{m}_{M} .
\end{aligned}
$$

Here $\mathbf{m}_{\tilde{L}}^{2}, \mathbf{m}_{\tilde{\nu}}^{2}$ and $\mathbf{A}_{\nu}$ are $3 \times 3$ soft SUSY-breaking matrices associated with the left-handed slepton doublets, the right-handed sneutrinos and their trilinear couplings, respectively. In the supersymmetric limit, all the soft SUSYbreaking matrices are equal to zero, $\tan \beta=1$ and $\mu=0$. As a consequence, the sneutrino mass matrix $\mathbf{M}_{\tilde{\nu}}^{2}$ can be expressed in terms of the neutrino mass matrix $\mathbf{M}_{\nu}$ in (2.3) as follows:

$$
\mathbf{M}_{\tilde{\nu}}^{2} \stackrel{\operatorname{SUSY}}{\longrightarrow}\left(\begin{array}{cc}
\mathbf{M}_{\nu} \mathbf{M}_{\nu}^{\dagger} & \mathbf{0}_{6 \times 6} \\
\mathbf{0}_{6 \times 6} & \mathbf{M}_{\nu}^{\dagger} \mathbf{M}_{\nu}
\end{array}\right),
$$

leading to the expected equality between neutrino and sneutrino mixings. Sneutrino LFV mixings do depend on the RG evolution of the $\nu_{R}$ MSSM parameters, but unlike the LFV mixings induced by soft SUSY-breaking terms, the sneutrino LFV mixings do not vanish at the GUT scale. 


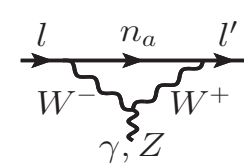

(a)

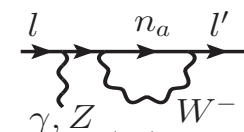

$\left(\mathbf{a}_{5}\right)$

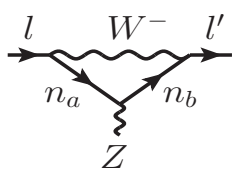

(a)

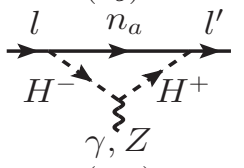

(a11)

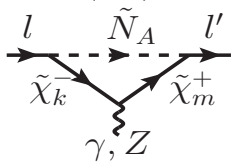

(a15)

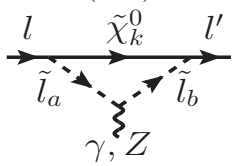

(a19)

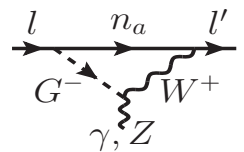

(a)

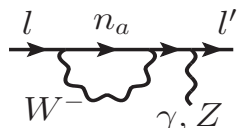

(a)

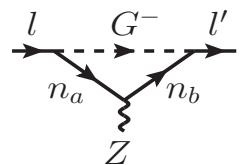

(a10)

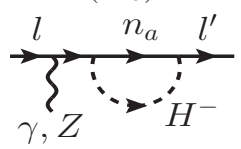

$\left(\mathbf{a}_{12}\right)$

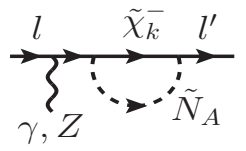

(a16)

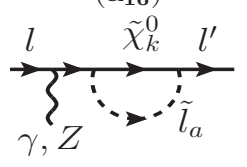

(a20)

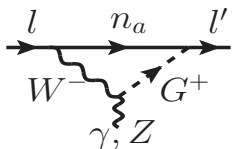

(a)

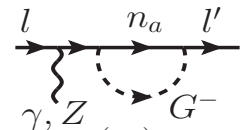

( $\left.\mathbf{a}_{7}\right)$

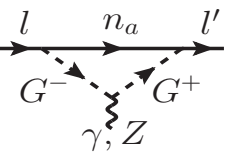

$\left(\mathbf{a}_{4}\right)$

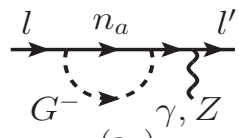

(as)

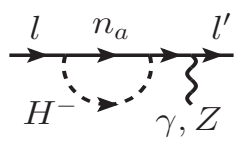

(a13)
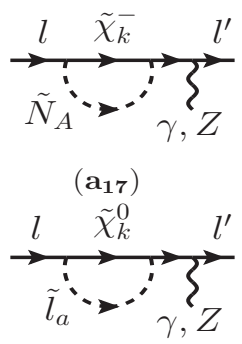

$\left(\mathbf{a}_{21}\right)$
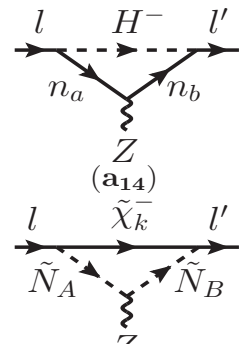

$Z$
$\left(\mathbf{a}_{18}\right)$

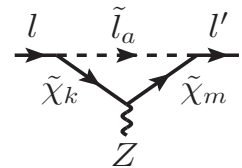

$\left(\mathbf{a}_{22}\right)$

FIG. 1: Feynman graphs contributing to $l \rightarrow l^{\prime} \gamma$ and $Z \rightarrow l^{C} l^{\prime}\left(l \rightarrow Z l^{\prime}\right)$ amplitudes

The sneutrino LFV mixings are obtained as combinations of unitary matrices that diagonalize the sneutrino, slepton and chargino mass matrices. It is interesting to notice that in the diagonalization of the sneutrino mass matrix $\mathbf{M}_{\tilde{\nu}}^{2}$ in (2.7), the sneutrino fields $\tilde{\nu}_{e, \mu, \tau L}, \tilde{\nu}_{1,2,3 R}^{C}$ and their complex conjugates $\tilde{\nu}_{e, \mu, \tau L}^{*}, \tilde{\nu}_{1,2,3 R}^{C *}$ are treated independently. As a result, the expressions for $\tilde{\nu}_{e, \mu, \tau L}$ and $\tilde{\nu}_{1,2,3 R}^{C}$, in terms of the real-valued mass eigenstates $\tilde{N}_{1,2, \ldots, 12}$, are not manifestly complex conjugates to $\tilde{\nu}_{e, \mu, \tau L}^{*}$ and $\tilde{\nu}_{1,2,3 R}^{C *}$, thus leading to a two-fold interpretation of the flavour basis fields,

$$
\begin{aligned}
& \tilde{\nu}_{i}^{*}=\left(\tilde{\nu}_{i}\right)^{*}=\mathcal{U}_{i A}^{\tilde{\nu} *} \widetilde{N}_{A}, \\
& \tilde{\nu}_{i}^{*}=\mathcal{U}_{i+6}^{\tilde{\nu}}{ }_{A} \widetilde{N}_{A}
\end{aligned}
$$

where $\tilde{\nu}_{1,2,3} \equiv \tilde{\nu}_{e, \mu, \tau L}$ and $\tilde{\nu}_{4,5,6} \equiv \tilde{\nu}_{1,2,3 R}^{C}$, with $i=1,2, \ldots, 6$ and $A=1,2, \ldots, 12$. For this reason, in Appendix $\mathrm{A}$ we include all equivalent forms that the Lagrangians, such as $\mathcal{L}_{\bar{e} \tilde{\chi}-\tilde{N}}$ and $\mathcal{L}_{\tilde{N} \tilde{N} Z}$, can be written down.

One technical comment is now in order. Unlike [34, 35], the diagonalization of the $12 \times 12$ sneutrino mass ma$\operatorname{trix} \mathbf{M}_{\tilde{\nu}}^{2}$ and the resulting interaction vertices are evaluated numerically, without approximations. To perform the diagonalization of $\mathbf{M}_{\tilde{\nu}}^{2}$ numerically, we use the method developed in [50] for the neutrino mass matrix. This method becomes very efficient, if the diagonal submatrices have eigenvalues larger than the eigenvalues of the other submatrices. Therefore, we assume that the heavy neutrino mass scale $m_{N}$ is of the order of, or larger than the scale of the other mass parameters in the $\nu_{R}$ MSSM.

Finally, a third source of LFV in the $\nu_{R}$ MSSM results from soft SUSY-breaking LFV terms. These LFV terms are induced by RG running and are absent at the GUT scale in mSUGRA. Their size strongly depends on the interval of the RG evolution from the GUT scale to the universal heavy neutrino mass scale $m_{N}$.

All the three different mechanisms of LFV, mediated by heavy neutrinos, heavy sneutrinos and soft SUSY-breaking terms, depend explicitly on the neutrino Yukawa matrix $\mathbf{h}_{\nu}$ and vanish in the limit $\mathbf{h}_{\nu} \rightarrow 0$. 


\section{CHARGED LEPTON FLAVOUR VIOLATION}

In this section, we present key details of our calculations for a number of CLFV observables in the $\nu_{R} \mathrm{MSSM}$. In detail, Section III A gives analytical results for the amplitudes of the CLFV decays: $l \rightarrow l^{\prime} \gamma$ and $Z \rightarrow l l^{\prime C}$, and their branching ratios. Correspondingly, Sections IIIB and IIIC give analytical expressions for the neutrinoless three-body decays $l \rightarrow l^{\prime} l_{1} l_{2}^{C}$ pertinent to muon and tau decays, and for coherent $\mu \rightarrow e$ conversion in nuclei. All these analytical results are expressed in terms of one-loop functions and composite formfactors that are defined in Appendices B and $\mathrm{C}$.

\section{A. The Decays $l \rightarrow l^{\prime} \gamma$ and $Z \rightarrow l l^{\prime C}$}

At the one-loop level, the effective couplings $\gamma l^{\prime} l$ and $Z l^{\prime} l$ are generated by the Feynman graphs shown in Fig. 1 . The general form of the transition amplitudes associated with these effective couplings is given by

$$
\begin{aligned}
\mathcal{T}_{\mu}^{\gamma l^{\prime} l}= & \frac{e \alpha_{w}}{8 \pi M_{W}^{2}} \bar{l}^{\prime}\left[\left(F_{\gamma}^{L}\right)_{l^{\prime} l}\left(q^{2} \gamma_{\mu}-\not q q_{\mu}\right) P_{L}+\left(F_{\gamma}^{R}\right)_{l^{\prime} l}\left(q^{2} \gamma_{\mu}-\not q q_{\mu}\right) P_{R}\right. \\
& \left.+\left(G_{\gamma}^{L}\right)_{l^{\prime} l} i \sigma_{\mu \nu} q^{\nu} P_{L}+\left(G_{\gamma}^{R}\right)_{l^{\prime} l} i \sigma_{\mu \nu} q^{\nu} P_{R}\right] l, \\
\mathcal{T}_{\mu}^{Z l^{\prime} l}= & \frac{g_{w} \alpha_{w}}{8 \pi \cos \theta_{w}} \bar{l}^{\prime}\left[\left(F_{Z}^{L}\right)_{l^{\prime} l} \gamma_{\mu} P_{L}+\left(F_{Z}^{R}\right)_{l^{\prime} l} \gamma_{\mu} P_{R}\right] l,
\end{aligned}
$$

where $P_{L(R)}=\frac{1}{2}\left[1-(+) \gamma_{5}\right], \alpha_{w}=g_{w}^{2} /(4 \pi), e$ is the electromagnetic coupling constant, $M_{W}=g_{w} \sqrt{v_{u}^{2}+v_{d}^{2}} / 2$ is the $W$-boson mass, $\theta_{w}$ is the weak mixing angle and $q=p_{l^{\prime}}-p_{l}$ is the photon momentum. The form factors $\left(F_{\gamma}^{L}\right)_{l^{\prime} l}$, $\left(F_{\gamma}^{R}\right)_{l^{\prime} l}\left(G_{\gamma}^{L}\right)_{l^{\prime} l},\left(G_{\gamma}^{R}\right)_{l^{\prime} l},\left(F_{Z}^{L}\right)_{l^{\prime} l}$ and $\left(F_{Z}^{R}\right)_{l^{\prime} l}$ receive contributions from heavy neutrinos $N_{1,2,3}$, heavy sneutrinos $\widetilde{N}_{1,2,3}$ and RG induced soft SUSY-breaking terms. The analytical expressions for these three individual contributions are given in Appendix C Note that according to our normalization, the composite formfactors $\left(G_{\gamma}^{L}\right)_{l^{\prime} l}$ and $\left(G_{\gamma}^{R}\right)_{l^{\prime} l}$ have dimensions of mass, whilst all other formfactors are dimensionless.

It is essential to remark here that the transition amplitudes (3.1) and (3.2) are also constituent parts of the leptonic amplitudes $l \rightarrow l^{\prime} l_{1} l_{2}^{C}$ and semileptonic amplitudes $l \rightarrow l^{\prime} q_{1} \bar{q}_{2}$, which will be discussed in more detail in Sections IIIB and IIIC. To calculate the CLFV decay $l \rightarrow l^{\prime} \gamma$, we only need to consider the dipole moment operators associated with the formfactors $\left(G_{\gamma}^{L}\right)_{l^{\prime} l}$ and $\left(G_{\gamma}^{R}\right)_{l^{\prime} l}$ in (3.1). Taking this last fact into account, the branching ratios for $l \rightarrow l^{\prime} \gamma$ and $Z \rightarrow l l^{\prime C}+l^{C} l^{\prime}$ are given by

$$
\begin{array}{r}
B\left(l \rightarrow l^{\prime} \gamma\right)=\frac{\alpha_{w}^{3} s_{w}^{2}}{256 \pi^{2}} \frac{m_{l}^{3}}{M_{W}^{4} \Gamma_{l}}\left(\left|\left(G_{\gamma}^{L}\right)_{l^{\prime} l}\right|^{2}+\left|\left(G_{\gamma}^{R}\right)_{l^{\prime} l}\right|^{2}\right), \\
B\left(Z \rightarrow l^{\prime} l^{C}+l^{\prime C} l\right)=\frac{\alpha_{w}^{3} M_{W}}{768 \pi^{2} c_{w}^{3} \Gamma_{Z}}\left(\left|\left(F_{Z}^{L}\right)_{l^{\prime} l}\right|^{2}+\left|\left(F_{Z}^{R}\right)_{l^{\prime} l}\right|^{2}\right) .
\end{array}
$$

Observe that the above expressions are valid to leading order in external charged lepton masses and external momenta, which constitutes an excellent approximation for our purposes. Thus, in (3.4) we have assumed that the $Z$-boson mass $M_{Z}$ is much smaller than the SUSY and heavy neutrino mass scales, $M_{\mathrm{SUSY}}$ and $m_{N}$, and we have kept the leading term in an expansion of small momenta and masses for the external particles. In the decoupling regime of all soft SUSY-breaking and charged Higgs-boson masses, the low-energy sector of the $\nu_{R}$ MSSM becomes the $\nu_{R}$ SM. In this $\nu_{R} \mathrm{SM}$ limit of the theory, the analytical expressions for $B\left(l \rightarrow l^{\prime} \gamma\right)$ and $B\left(Z \rightarrow l^{\prime} l^{C}+l^{\prime C} l\right)$ take on the forms presented in [51] and [47], respectively.

\section{B. Three-Body Leptonic Decays $l \rightarrow l^{\prime} l_{1} l_{2}^{C}$}

We now study the three-body CLFV decays $l \rightarrow l^{\prime} l_{1} l_{2}^{C}$, where $l$ can be the muon or tau, and $l^{\prime}, l_{1}, l_{2}$ denote other charged leptons to which $l$ is allowed to decay kinematically.

The transition amplitude for $l \rightarrow l^{\prime} l_{1} l_{2}^{C}$ receives contributions from $\gamma$ - and $Z$-boson-mediated graphs shown in Fig. 1 and from box graphs displayed in Fig. 2. The amplitudes for these three contributions are

$$
\mathcal{T}_{\gamma}^{l l^{\prime} l_{1} l_{2}}=\frac{\alpha_{w}^{2} s_{w}^{2}}{2 M_{W}^{2}}\left\{\delta _ { l _ { 1 } l _ { 2 } } \overline { l } ^ { \prime } \left[\left(F_{\gamma}^{L}\right)_{l^{\prime} l} \gamma_{\mu} P_{L}+\left(F_{\gamma}^{R}\right)_{l^{\prime} l} \gamma_{\mu} P_{R}\right.\right.
$$




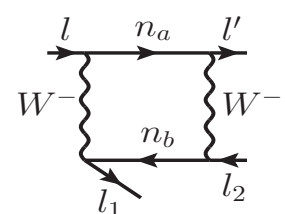

(a)

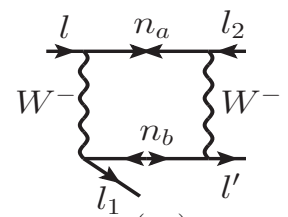

(as)

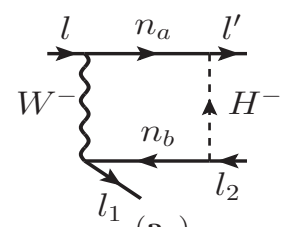

(a9)
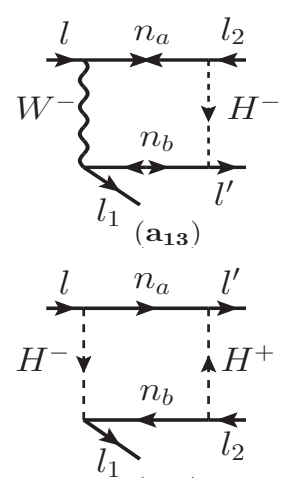

$\left(\mathbf{a}_{17}\right)$

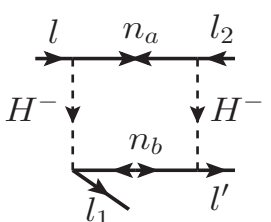

(a18)

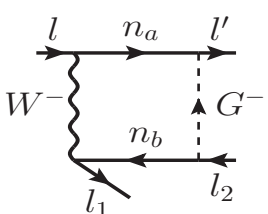

$\left(\mathbf{a}_{2}\right)$

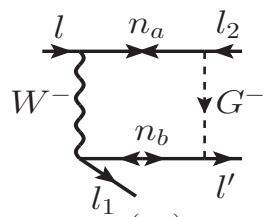

( $\left.\mathbf{a}_{6}\right)$

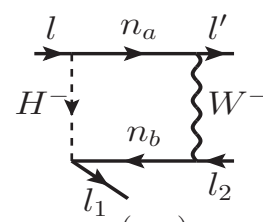

(a10)
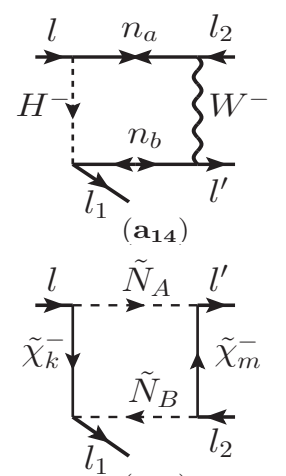

(a19)

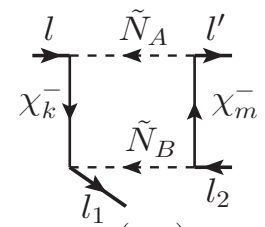

(a.21)

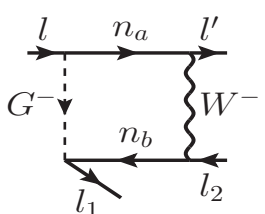

$\left(\mathbf{a}_{3}\right)$

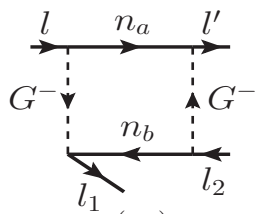

$\left(\mathbf{a}_{4}\right)$

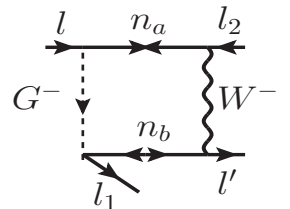

(a)

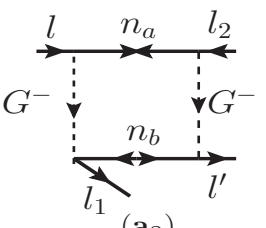

(a)

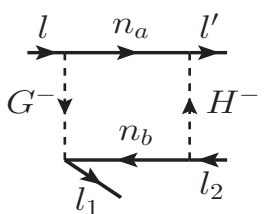

(a11)

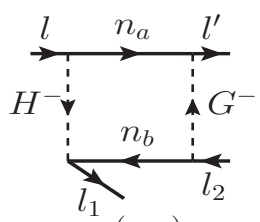

(a12)

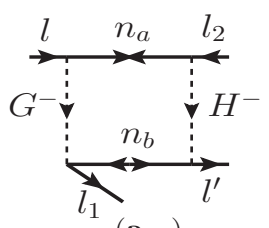

(a15)
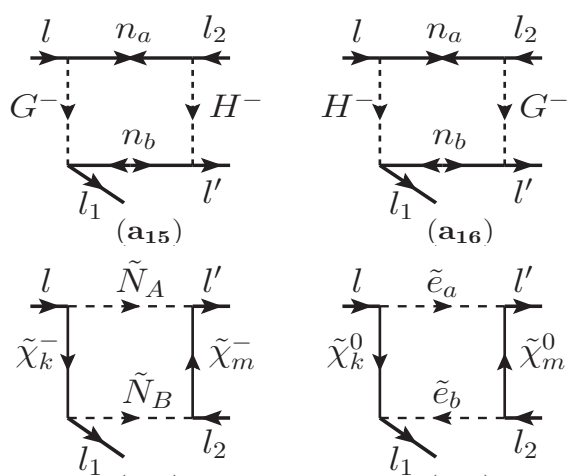

(a)

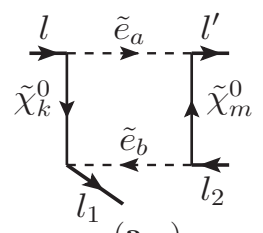

(a $\left.\mathbf{a}_{23}\right)$

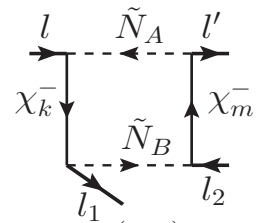

(a22)

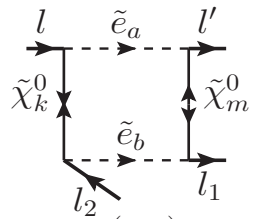

(a $\left.\mathbf{a}_{24}\right)$

$+\left(l^{\prime} \leftrightarrow l_{1}\right)$

FIG. 2: Feynman graphs contributing to the box $l \rightarrow l^{\prime} l_{1} l_{2}^{C}$ amplitudes.

$$
\begin{aligned}
& \left.\left.+\frac{\left(\not p-\not p^{\prime}\right)}{\left(p-p^{\prime}\right)^{2}}\left(\left(G_{\gamma}^{L}\right)_{l^{\prime} l} \gamma_{\mu} P_{L}+\left(G_{\gamma}^{R}\right)_{l^{\prime} l} \gamma_{\mu} P_{R}\right)\right] l \bar{l}_{1} \gamma^{\mu} l_{2}^{C}-\left[l^{\prime} \leftrightarrow l_{1}\right]\right\} \\
\mathcal{T}_{Z}^{l l^{\prime} l_{1} l_{2}=}= & \frac{\alpha_{w}^{2}}{2 M_{W}^{2}}\left[\delta_{l_{1} l_{2}} \bar{l}^{\prime}\left(\left(F_{Z}^{L}\right)_{l^{\prime} l} \gamma_{\mu} P_{L}+\left(F_{Z}^{R}\right)_{l^{\prime} l} \gamma_{\mu} P_{R}\right) l \bar{l}_{1}\left(g_{L}^{l} \gamma^{\mu} P_{L}+g_{R}^{l} \gamma^{\mu} P_{R}\right) l_{2}^{C}-\left(l^{\prime} \leftrightarrow l_{1}\right)\right], \\
\mathcal{T}_{\text {box }}^{l l^{\prime} l_{1} l_{2}}= & -\frac{\alpha_{w}^{2}}{4 M_{W}^{2}}\left(B_{\ell V}^{L L} \bar{l}^{\prime} \gamma_{\mu} P_{L} l \bar{l}_{1} \gamma^{\mu} P_{L} l_{2}^{C}+B_{\ell V}^{R R} \bar{l}^{\prime} \gamma_{\mu} P_{R} l \bar{l}_{1} \gamma^{\mu} P_{R} l_{2}^{C}\right. \\
& +B_{\ell V}^{L R} \bar{l}^{\prime} \gamma_{\mu} P_{L} l \bar{l}_{1} \gamma^{\mu} P_{R} l_{2}^{C}+B_{\ell V}^{R L} \bar{l}^{\prime} \gamma_{\mu} P_{R} l \bar{l}_{1} \gamma^{\mu} P_{L} l_{2}^{C} \\
& +B_{\ell S}^{L L} \bar{l}^{\prime} P_{L} l \bar{l}_{1} P_{L} l_{2}^{C}+B_{\ell S}^{R R} \bar{l}^{\prime} P_{R} l \bar{l}_{1} P_{R} l_{2}^{C}+B_{\ell S}^{L R} \bar{l}^{\prime} P_{L} l \bar{l}_{1} P_{R} l_{2}^{C}+B_{\ell S}^{R L} \bar{l}^{\prime} P_{R} l \bar{l}_{1} P_{L} l_{2}^{C} \\
& \left.+B_{\ell T}^{L L} \bar{l}^{\prime} \sigma_{\mu \nu} P_{L} l \bar{l}_{1} \sigma^{\mu \nu} P_{L} l_{2}^{C}+B_{\ell T}^{R R} \bar{l}^{\prime} \sigma_{\mu \nu} P_{R} l \bar{l}_{1} \sigma^{\mu \nu} P_{R} l_{2}^{C}\right)
\end{aligned}
$$




$$
\equiv-\frac{\alpha_{w}^{2}}{4 M_{W}^{2}} \sum_{X, Y=L, R} \sum_{A=V, S, T} B_{\ell A}^{X Y} \bar{l}^{\prime} \Gamma_{A}^{X} l \bar{l}_{1} \Gamma_{A}^{Y} l_{2}^{C}
$$

where $g_{L}^{l}=-1 / 2+s_{w}^{2}$ and $g_{R}^{l}=s_{w}^{2}$ are $Z$-boson-lepton couplings and $s_{w}=\sin \theta_{w}$. The composite box formfactors $B_{\ell A}^{X Y}, A=V, S, T, X, Y=L, R$ are given in Appendix C. The labels $V, S$ and $T$ denote the formfactors of the vector, scalar and tensor combinations of the currents, while $L$ and $R$ distinguish between left and right chiralities of these currents. We note that the box formfactors contain both direct and Fierz-transformed contributions. Equation (3.8) represents a shorthand expression that takes account of all individual contributions to the amplitude $\mathcal{T}_{\text {box }}^{l l^{\prime} l_{1} l_{2}}$ induced by box graphs. Explicitly, the matrices $\Gamma_{A}^{X}$ appearing in (3.8) read:

$$
\left(\Gamma_{V}^{L}, \Gamma_{V}^{R}, \Gamma_{S}^{L}, \Gamma_{S}^{R}, \Gamma_{T}^{L}, \Gamma_{T}^{R}\right)=\left(\gamma_{\mu} P_{L}, \gamma_{\mu} P_{R}, P_{L}, P_{R}, \sigma_{\mu \nu} P_{L}, \sigma_{\mu \nu} P_{R}\right)
$$

Notice that the tensor formfactors $B_{\ell T}^{L R}$ and $B_{\ell T}^{R L}$ vanish in the sum (3.8), i.e. $B_{\ell T}^{L R}=B_{\ell T}^{R L}=0$, as a consequence of the identity $\sigma^{\mu \nu} \gamma_{5}=-\frac{i}{2} \varepsilon^{\mu \nu \rho \tau} \sigma_{\rho \tau}$. A very similar chiral structure holds also true for the semileptonic box amplitudes defined in the next section. We should remark here that [37] and [52] do not include in their calculations the chiral structures $P_{L} \times P_{R}$ and $P_{R} \times P_{L}$ and their corresponding formfactors $B_{\ell S}^{L R}$ and $B_{\ell S}^{R L}$.

In a three-generation model, the transition amplitude for the decays $l \rightarrow l^{\prime} l_{1} l_{2}^{C}$ may fall in one of the following three classes or categories [33]: (i) $l^{\prime} \neq l_{1}=l_{2}$, (ii) $l^{\prime}=l_{1}=l_{2}$, and (iii) $l^{\prime}=l_{1} \neq l_{2}$. In the first two classes (i) and (ii), the total lepton number is conserved, whilst in the third class (iii) the total lepton number is violated by two units. Here, we ignore this lepton-number violating class (iii) of charged lepton decays, since the predictions turn out to be unobservably small in the $\nu_{R}$ MSSM. Moreover, we suppress the universal indices $l^{\prime} l$ that appear in the photon and $Z$-boson formfactors, i.e. $F_{\gamma}^{L}, F_{\gamma}^{R}, F_{Z}^{L}$ and $F_{Z}^{R}$. Given the above simplification and the notation of the box formfactors in (3.8), the branching ratios for the class (i) and (ii) of CLFV three-body decays are given by

$$
\begin{aligned}
B\left(l \rightarrow l^{\prime} l_{1} l_{1}^{C}\right)= & \frac{m_{l}^{5} \alpha_{w}^{4}}{24576 \pi^{3} M_{W}^{4} \Gamma_{l}}\left\{\left[\left|2 s_{w}^{2}\left(F_{\gamma}^{L}+F_{Z}^{L}\right)-F_{Z}^{L}-B_{\ell V}^{L L}\right|^{2}+\left|2 s_{w}^{2}\left(F_{\gamma}^{R}+F_{Z}^{R}\right)-B_{\ell V}^{R R}\right|^{2}\right.\right. \\
& \left.+\left|2 s_{w}^{2}\left(F_{\gamma}^{L}+F_{Z}^{L}\right)-B_{\ell V}^{L R}\right|^{2}+\left|2 s_{w}^{2}\left(F_{\gamma}^{R}+F_{Z}^{R}\right)-F_{Z}^{R}-B_{\ell V}^{R L}\right|^{2}\right] \\
& +\frac{1}{4}\left(\left|B_{\ell S}^{L L}\right|^{2}+\left|B_{\ell S}^{R R}\right|^{2}+\left|B_{\ell S}^{L R}\right|^{2}+\left|B_{\ell S}^{R L}\right|^{2}\right)+12\left(\left|B_{\ell T}^{L L}\right|^{2}+\left|B_{\ell T}^{R R}\right|^{2}\right) \\
& +\frac{32 s_{w}^{4}}{m_{l}}\left[\operatorname{Re}\left(\left(F_{\gamma}^{R}+F_{Z}^{R}\right) G_{\gamma}^{L *}\right)+\operatorname{Re}\left(\left(F_{\gamma}^{L}+F_{Z}^{L}\right) G_{\gamma}^{R *}\right)\right] \\
& -\frac{8 s_{w}^{2}}{m_{l}}\left[\operatorname{Re}\left(\left(F_{Z}^{R}+B_{\ell V}^{R R}+B_{\ell V}^{R L}\right) G_{\gamma}^{L *}\right)+\operatorname{Re}\left(\left(F_{Z}^{L}+B_{\ell V}^{L L}+B_{\ell V}^{L R}\right) G_{\gamma}^{R *}\right)\right] \\
& \left.+\frac{32 s_{w}^{4}}{m_{l}^{2}}\left(\left|G_{\gamma}^{L}\right|^{2}+\left|G_{\gamma}^{R}\right|^{2}\right)\left(\ln \frac{m_{l}^{2}}{m_{l^{\prime}}^{2}}-3\right)\right\}, \\
B\left(l \rightarrow l^{\prime} l^{\prime} l^{\prime C}\right)= & \frac{m_{l}^{5} \alpha_{w}^{4}}{24576 \pi^{3} M_{W}^{4} \Gamma_{l}}\left\{2\left[\left|2 s_{w}^{2}\left(F_{\gamma}^{L}+F_{Z}^{L}\right)-F_{Z}^{L}-\frac{1}{2} B_{\ell V}^{L L}\right|^{2}+\left|2 s_{w}^{2}\left(F_{\gamma}^{R}+F_{Z}^{R}\right)-\frac{1}{2} B_{\ell V}^{R R}\right|^{2}\right]\right. \\
& +\left|2 s_{w}^{2}\left(F_{\gamma}^{L}+F_{Z}^{L}\right)-B_{\ell V}^{L R}\right|^{2}+\left|2 s_{w}^{2}\left(F_{\gamma}^{R}+F_{Z}^{R}\right)-\left(F_{Z}^{R}+B_{\ell V}^{R L}\right)\right|^{2}+\frac{1}{8}\left(\left|B_{\ell S}^{L L}\right|^{2}+\left|B_{\ell S}^{R R}\right|^{2}\right) \\
& +6\left(\left|B_{\ell T}^{L L}\right|^{2}+\left|B_{\ell T}^{R R}\right|^{2}\right)+\frac{48 s_{w}^{4}}{m_{l}}\left[\operatorname{Re}\left(\left(F_{\gamma}^{R}+F_{Z}^{R}\right) G_{\gamma}^{L *}\right)+\operatorname{Re}\left(\left(F_{\gamma}^{L}+F_{Z}^{L}\right) G_{\gamma}^{R *}\right)\right] \\
& -\frac{8 s_{w}^{2}}{m_{l}}\left[\operatorname{Re}\left(\left(F_{Z}^{R}+B_{\ell V}^{R R}+B_{\ell V}^{R L}\right) G_{\gamma}^{L *}\right)+\operatorname{Re}\left(\left(2 F_{Z}^{L}+B_{\ell V}^{L L}+B_{\ell V}^{L R}\right) G_{\gamma}^{R *}\right)\right] \\
& \left.+\frac{32 s_{w}^{4}}{m_{l}^{2}}\left(\left|G_{\gamma}^{L}\right|^{2}+\left|G_{\gamma}^{R}\right|^{2}\right)\left(\ln \frac{m_{l}^{2}}{m_{l^{\prime}}^{2}}-\frac{11}{4}\right)\right\},
\end{aligned}
$$

where $m_{l}$ and $m_{l^{\prime}}, m_{l_{1}}, m_{l_{2}}$ are the masses of the initial- and final-state charged leptons and $\Gamma_{l}$ is the decay width of the charged lepton $l$. Here we should emphasize that the transition amplitudes (3.5), (3.6), (3.7) and the branching ratios (3.10) and (3.11) have the most general chiral and formfactor structure to leading order in external masses and momenta and so they are applicable to most models of New Physics with CLFV. Finally, we have checked that the branching ratios (3.10) and (3.11) go over to the results of [33], in the $\nu_{R} \mathrm{SM}$ limit of the theory. 


\section{Coherent $\mu \rightarrow e$ Conversion in a Nucleus}

The coherent $\mu \rightarrow e$ conversion in a nucleus corresponds to the process $J_{\mu} \rightarrow e^{-} J^{+}$, where $J_{\mu}$ is an atom of nucleus $J$ with one orbital electron replaced by a muon and $J^{+}$is the corresponding ion without the muon. The transition amplitude for such a CLFV process,

$$
\mathcal{T}^{\mu e ; J}=\left\langle J^{+} e^{-}\left|\mathcal{T}^{d \mu \rightarrow d e}\right| J_{\mu}\right\rangle+\left\langle J^{+} e^{-}\left|\mathcal{T}^{u \mu \rightarrow u e}\right| J_{\mu}\right\rangle,
$$

depends on two effective box operators,

$$
\begin{aligned}
\mathcal{T}_{\text {box }}^{d \mu \rightarrow d e} & =-\frac{\alpha_{w}^{2}}{4 M_{W}^{2}} \sum_{X, Y=L, R} \sum_{A=V, S, T} B_{d A}^{X Y} \bar{e} \Gamma_{A}^{X} \mu \bar{d} \Gamma_{A}^{X} d \\
& =-\frac{\alpha_{w}^{2}}{2 M_{W}^{2}}\left(d^{\dagger} d\right) \bar{e}\left(V_{d}^{R} P_{R}+V_{d}^{L} P_{L}\right) \mu, \\
\mathcal{T}_{\text {box }}^{u \mu \rightarrow u e} & =-\frac{\alpha_{w}^{2}}{4 M_{W}^{2}} \sum_{X, Y=L, R} \sum_{A=V, S, T} B_{u A}^{X Y} \bar{e} \Gamma_{A}^{X} \mu \bar{u} \Gamma_{A}^{X} u \\
& =-\frac{\alpha_{w}^{2}}{2 M_{W}^{2}}\left(u^{\dagger} u\right) \bar{e}\left(V_{u}^{R} P_{R}+V_{u}^{L} P_{L}\right) \mu .
\end{aligned}
$$

Here $\mu$ and $e$ are the muon and electron wave functions and $d$ and $u$ are field operators acting on the $J_{\mu}$ and $J^{+}$ states. The formfactors $B_{d A}^{X Y}$ and $B_{u A}^{X Y}$ are given in Appendix [C] The composite formfactors $V_{d}^{L}, V_{u}^{L}, V_{d}^{R}, V_{u}^{R}$ may conveniently be expressed as

$$
\begin{aligned}
V_{d}^{L} & =-\frac{1}{3} s_{w}^{2}\left(F_{\gamma}^{L}-\frac{1}{m_{\mu}} G_{\gamma}^{R}\right)+\left(\frac{1}{4}-\frac{1}{3} s_{w}^{2}\right) F_{Z}^{L}+\frac{1}{4}\left(B_{d V}^{L L}+B_{d V}^{L R}+B_{d S}^{R R}+B_{d S}^{R L}\right), \\
V_{d}^{R} & =-\frac{1}{3} s_{w}^{2}\left(F_{\gamma}^{R}-\frac{1}{m_{\mu}} G_{\gamma}^{L}\right)+\left(\frac{1}{4}-\frac{1}{3} s_{w}^{2}\right) F_{Z}^{R}+\frac{1}{4}\left(B_{d V}^{R R}+B_{d V}^{R L}+B_{d S}^{L L}+B_{d S}^{L R}\right), \\
V_{u}^{L} & =\frac{2}{3} s_{w}^{2}\left(F_{\gamma}^{L}-\frac{1}{m_{\mu}} G_{\gamma}^{R}\right)+\left(-\frac{1}{4}+\frac{2}{3} s_{w}^{2}\right) F_{Z}^{L}+\frac{1}{4}\left(B_{u V}^{L L}+B_{u V}^{L R}+B_{u S}^{R R}+B_{u S}^{R L}\right), \\
V_{u}^{R} & =\frac{2}{3} s_{w}^{2}\left(F_{\gamma}^{R}-\frac{1}{m_{\mu}} G_{\gamma}^{L}\right)+\left(-\frac{1}{4}+\frac{2}{3} s_{w}^{2}\right) F_{Z}^{R}+\frac{1}{4}\left(B_{u V}^{R R}+B_{u V}^{R L}+B_{u S}^{L L}+B_{u S}^{L R}\right),
\end{aligned}
$$

where $F_{\gamma}^{L}, F_{\gamma}^{R}, F_{Z}^{L}, F_{Z}^{R}$ is the shorthand notation for $\left(F_{\gamma}^{L}\right)_{e \mu},\left(F_{\gamma}^{R}\right)_{e \mu},\left(F_{Z}^{L}\right)_{e \mu},\left(F_{Z}^{R}\right)_{e \mu}$.

Our next step is to determine the nucleon matrix elements of the operators $u^{\dagger} u$ and $d^{\dagger} d$. These are given by

$$
\begin{aligned}
\left\langle J^{+} e^{-}\left|u^{\dagger} u\right| J_{\mu}\right\rangle & =(2 Z+N) F\left(-m_{\mu}^{2}\right), \\
\left\langle J^{+} e^{-}\left|d^{\dagger} d\right| J_{\mu}\right\rangle & =(Z+2 N) F\left(-m_{\mu}^{2}\right),
\end{aligned}
$$

where the formfactor $F\left(q^{2}\right)$ incorporates the recoil of the $J^{+}$ion [53], and the factors $2 Z+N$ and $Z+2 N$ count the number of $u$ and $d$ quarks in the nucleus $J$, respectively. Hence, the matrix element for $J_{\mu} \rightarrow J^{+} \mu^{-}$can be written down as

$$
T^{J_{\mu} \rightarrow J^{+} e^{-}}=-\frac{\alpha_{w}^{2}}{2 M_{W}^{2}} F\left(-m_{\mu}^{2}\right) \bar{e}\left(Q_{W}^{L} P_{R}+Q_{W}^{R} P_{L}\right) \mu
$$

with

$$
\begin{aligned}
& Q_{W}^{L}=(2 Z+N) V_{u}^{L}+(Z+2 N) V_{d}^{L}, \\
& Q_{W}^{R}=(2 Z+N) V_{u}^{R}+(Z+2 N) V_{d}^{R} .
\end{aligned}
$$

Given the transition amplitude (3.17), the decay rate $J_{\mu} \rightarrow J^{+} e^{-}$is found to be

$$
R_{\mu e}^{J}=\frac{\alpha^{3} \alpha_{w}^{4} m_{\mu}^{5}}{16 \pi^{2} M_{W}^{4} \Gamma_{\text {capture }}} \frac{Z_{\text {eff }}^{4}}{Z}\left|F\left(-m_{\mu}^{2}\right)\right|^{2}\left(\left|Q_{W}^{L}\right|^{2}+\left|Q_{W}^{R}\right|^{2}\right),
$$

where $\Gamma_{\text {capture }}$ is the capture rate of the muon by the nucleus, and $Z_{\text {eff }}$ is the effective charge which takes into account coherent effects that can occur in the nucleus $J$ due to its finite size. In our analysis, we use the values of $Z_{\text {eff }}$ quoted in [54]. We reiterate that the branching ratio (3.19) possesses the most general formfactor structure to leading order in external masses and momenta and is relevant to most models of New Physics with CLFV. Finally, we have verified that our analytical results are consistent with [34, [55, [56] in the $\nu_{R} \mathrm{SM}$ limit of the theory. 


\section{NUMERICAL RESULTS}

In this section, we present a numerical analysis of CLFV observables in the $\nu_{R} \mathrm{MSSM}$. In order to reduce the number of independent parameters, we adopt the constrained framework of mSUGRA. In detail, our model parameters are: (i) the usual SM parameters, such as gauge coupling constants, the quark and charged-lepton Yukawa matrices inputted at the scale $M_{Z}$, (ii) the heavy neutrino mass $m_{N}$ and the neutrino Yukawa matrix $\mathbf{h}_{\nu}$ evaluated at $m_{N}$, (iii) the universal mSUGRA parameters $m_{0}, M_{1 / 2}$ and $A_{0}$ inputted at the GUT scale, and (iv) the ratio tan $\beta$ of the Higgs VEVs and the sign of the superpotential Higgs-mixing parameter $\mu$.

The allowed ranges of the soft SUSY-breaking parameters $m_{0}, M_{1 / 2}, A_{0}$ and $\tan \beta$ are strongly constrained by a number of accelerator and cosmological data [21, 22, 46, 57]. For definiteness, we consider the following set of input parameters:

$$
\tan \beta=10, \quad m_{0}=1000 \mathrm{GeV}, \quad A_{0}=-3000 \mathrm{GeV}, \quad M_{1 / 2}=1000 \mathrm{GeV} .
$$

Here we take the $\mu$ parameter to be positive, whilst its absolute value $|\mu|$ is derived form the minimization of the Higgs potential at the scale $M_{Z}$. With aid of [58 60], we verify that the parameter set (4.1) predicts a SM-like Higgs boson with $m_{H} \approx 125 \mathrm{GeV}$, in agreement with the recent discovery at the LHC [21, 22], and is compatible with the current lower limits on gluino and squark masses [46]. The set (4.1) is also in agreement with having the lightest neutralino as the Dark Matter in the Universe [57].

We employ the one-loop RG equations of [61, 62] to evolve the the gauge coupling constants and the quark and charged lepton Yukawa matrices from $M_{Z}$ to the GUT scale, while the heavy neutrino mass matrix $\mathbf{m}_{M}$ and the neutrino Yukawa matrix $\mathbf{h}_{\nu}$ are evolved from the heavy neutrino mass threshold $m_{N}$ to the GUT scale. Furthermore, we assume that the heavy neutrino-sneutrino sector is supersymmetric above $m_{N}$. For purposes of RG evolution, this is a good approximation for $m_{N}$ larger than the typical soft SUSY-breaking scale [34]. At the GUT scale, the mSUGRA universality conditions are used to express the soft SUSY-breaking masses, in terms of $m_{0}, M_{1 / 2}$ and $A_{0}$. Hence, all scalar masses receive a soft SUSY-breaking mass $m_{0}$, all gaugino are mass-degenerate to $M_{1 / 2}$, and all scalar trilinear couplings are of the form $\mathbf{h}_{x} A_{0}$, with $x=u, d, l, \nu$, where $\mathbf{h}_{x}$ are the Yukawa matrices at the GUT scale. The sneutrino mass matrix acquires additional contributions from the heavy neutrino mass matrix. The sparticle mass matrices and trilinear couplings are evolved from the GUT scale to $M_{Z}$, except for the sneutrino masses which are evolved to the heavy neutrino threshold $m_{N}$. Having thus obtained all sparticle and sneutrino mass matrices, we can numerically evaluate all particle masses and interaction vertices in the $\nu_{R}$ MSSM, without approximations.

To simplify our numerical analysis, we consider two representative scenarios of Yukawa textures discussed in Section II Specifically, the first scenario realizes the U(1)-symmetric Yukawa texture in (2.4), for which we take either $a=b$ and $c=0$, or $a=c$ and $b=0$, or $b=c$ and $a=0$, thus giving rise to CLFV processes $\mu \rightarrow e X, \tau \rightarrow e X$ and $\tau \rightarrow \mu X$, respectively. Here $X$ represents the lepton-flavour conserving state(s), e.g. $X=\gamma, e^{+} e^{-}, \mu^{+} \mu^{-}, q \bar{q}$. The second scenario is motivated by the $A_{4}$ group and uses the Yukawa texture (2.5), where the parameters $a, b$ and $c$ are taken to be all equal, i.e. $a=b=c$.

The heavy neutrino mass scale $m_{N}$ strongly depends on the size of the symmetry-breaking terms in the Yukawa matrix $\mathbf{h}_{\nu}$. For instance, for the model (2.4), the typical values of the $U(1)$-lepton-symmetry-breaking parameters $\epsilon_{l} \equiv \epsilon_{e, \mu, \tau}$ consistent with low-scale resonant leptogenesis is $\epsilon \lesssim 10^{-5}$ [30], leading to light-neutrino masses

$$
m_{\nu} \sim \frac{\epsilon_{l}^{2} v^{2}}{m_{N}} \sim 10^{-2} \mathrm{eV}\left(\frac{\epsilon_{l}}{10^{-6}}\right)^{2}\left(\frac{1 \mathrm{TeV}}{m_{N}}\right)
$$

Taking into account the constraint $m_{\nu} \gtrsim 10^{-1} \mathrm{eV}$ generically derived from neutrino oscillation data, we may estimate that the heavy neutrino mass scale $m_{N}$ is typically restricted to be less than $10 \mathrm{TeV}$, for $\epsilon_{l}=10^{-5}$. If the assumption of successful low-scale leptogenesis is relaxed, the symmetry-breaking parameters $\epsilon_{l}$ has only to be couple of orders in magnitude smaller than the Yukawa parameters $a, b$ and $c$, with $a, b, c \lesssim 10$. Thus, for $\epsilon_{l}<10^{-3}-10^{-2}$, the heavy neutrino mass scale $m_{N}$ may be as large as $10^{7}-10^{9} \mathrm{TeV}$, leading to the decoupling of heavy neutrinos from low-energy observables. As our interest is in the interplay between heavy neutrino, sneutrino and soft SUSY-breaking contributions to CLFV observables, we will only study here the parameter space in which $m_{N}<10 \mathrm{TeV}$.

In the present analysis, we consider that the symmetry-preserving Yukawa parameters $a, b$ and $c$ are limited through the perturbativity condition: $\operatorname{Tr} \mathbf{h}_{\nu}^{\dagger} \mathbf{h}_{\nu}<4 \pi$, which we require to hold true for the entire interval of the RG evolution: $\ln \left(M_{Z} / \mathrm{TeV}\right)<t<\ln \left(M_{\mathrm{GUT}} / \mathrm{TeV}\right)$. For the model in (2.4), this condition translates into the constraint: $a<0.34$, and for the model in (2.5), to $a<0.23$. As a consequence, we do not display in plots numerical values for points in parameter space, for which the aforementioned perturbativity condition gets violated.

In Fig. 3, we display numerical predictions for the $\mu$-LFV observables $B(\mu \rightarrow e X): B(\mu \rightarrow e \gamma)$ [blue (solid) line], $B(\mu \rightarrow$ eee $)$ [red (dashed) line], $R_{\mu e}^{\mathrm{Ti}}$ [violet (dotted) line] and $R_{\mu e}^{\mathrm{Au}}$ [green (dash-dotted) line], as functions of $B(\mu \rightarrow e \gamma)$ 

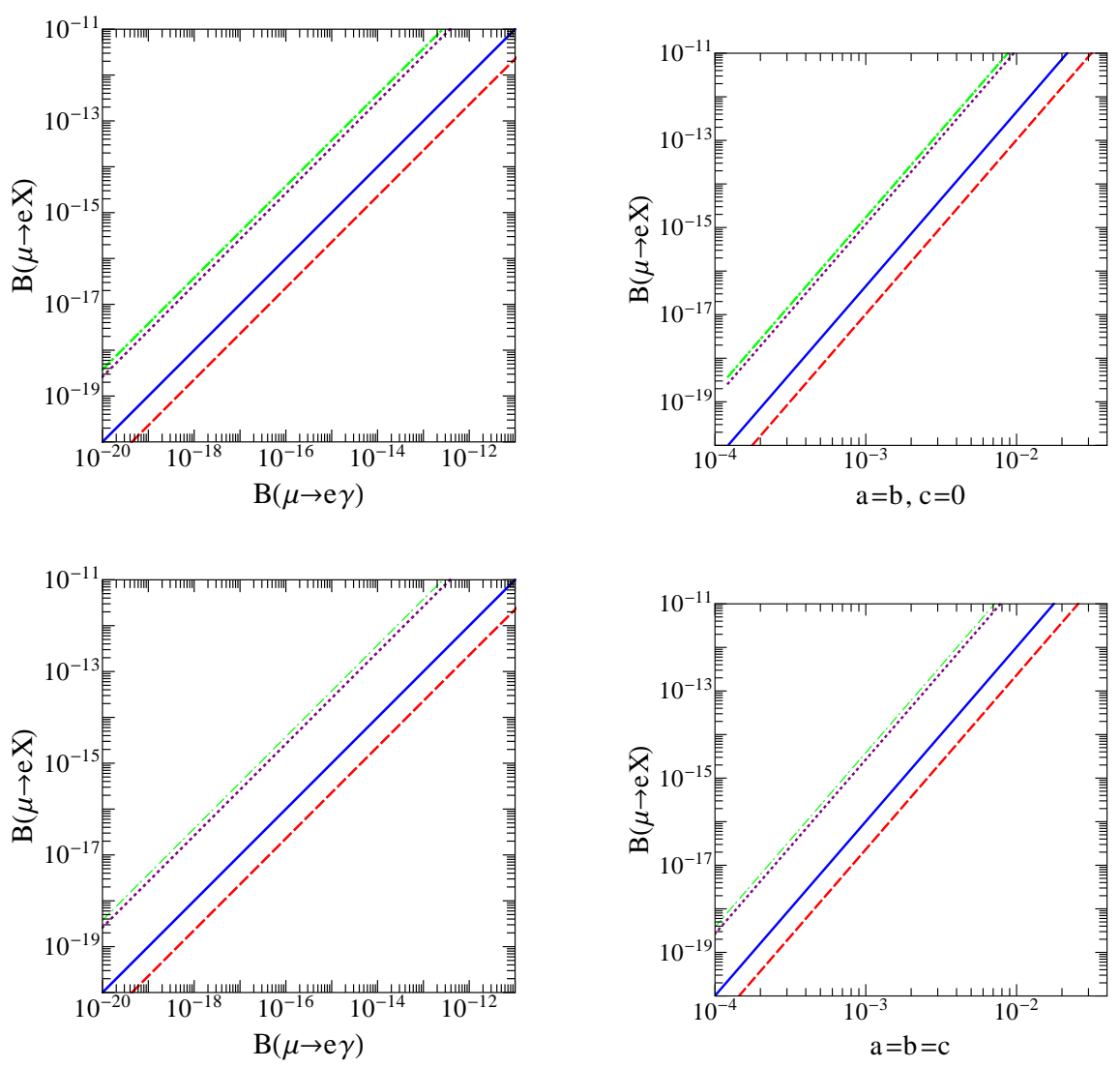

FIG. 3: Numerical estimates of $B(\mu \rightarrow e \gamma)$ [blue (solid)], $B(\mu \rightarrow e e e)$ [red (dashed)], $R_{\mu e}^{\mathrm{Ti}}$ [violet (dotted)] and $R_{\mu e}^{\mathrm{Au}}$ [green (dash-dotted)], as functions of $B(\mu \rightarrow e \gamma)$ (left pannels) and the Yukawa parameter $a$ (right pannels), for $m_{N}=400 \mathrm{GeV}$ and $\tan \beta=10$. The upper two pannels correspond to the Yukawa texture (2.4), with $a=b$ and $c=0$, and the lower two pannels to the Yukawa texture (2.5), with $a=b=c$.

(left pannels) and the Yukawa parameter $a$ (right pannels), for $m_{N}=400 \mathrm{GeV}$ and $\tan \beta=10$. The upper two pannels assume the Yukawa texture in (2.4), with $a=b$ and $c=0$, whilst the lower two pannels correspond to the Yukawa texture in (2.5), with $a=b=c$. In Fig. 4 we give numerical estimates for the same set of $\mu$-LFV observables, but for $m_{N}=1 \mathrm{TeV}$. In Figs. 3 and 4 the Yukawa parameter $a$ has been chosen, such that $10^{-20}<B(\mu \rightarrow e \gamma)<10^{-10}$. Such a range of values includes both the present [7-10, 63, 64] and future [16, 17, 19, 65 68] experimental limits. As we see from Figs. 3 and 4, the CLFV observables under study depend quadratically on the Yukawa parameter $a$, namely they are proportional to $a^{2}$. Instead, the quartic Yukawa terms proportional to $a^{4}[34$ remain always small, which is a consequence of the imposed perturbativity constraint: $\operatorname{Tr}\left(\mathbf{h}_{\nu}^{\dagger} \mathbf{h}_{\nu}\right)<4 \pi$, up to the GUT scale.

By analogy, Figs. 5 and 6 present numerical estimates of the $\tau$-LFV observables $B(\tau \rightarrow e X): B(\tau \rightarrow e \gamma)$ [blue (solid) lines], $B(\tau \rightarrow e e e)$ [red (dashed) lines] and $B(\tau \rightarrow e \mu \mu)$ [violet (dotted) lines], as functions of $B(\tau \rightarrow e \gamma)$ (left pannels) and the Yukawa parameter $a$ (right pannels), for $m_{N}=400 \mathrm{GeV}$ and $m_{N}=1 \mathrm{TeV}$, respectively. Note that we do not show predictions for the fully complementary observables $B(\tau \rightarrow \mu X): B(\tau \rightarrow \mu \gamma), B(\tau \rightarrow \mu \mu \mu)$ and $B(\tau \rightarrow \mu e e)$. The upper pannels give our predictions for the Yukawa texture (2.4), with $a=c$ and $b=0$, and the lower pannels for the Yukawa texture (2.5), with $a=b=c$. In both Figs. 5 and 6, the Yukawa parameter $a$ has been chosen, such that $10^{-16}<B(\tau \rightarrow e \gamma)<10^{-7}$. As can be seen from Figs. [5 and 6, all observables $B(\tau \rightarrow e X)$ of $\tau$-LFV (with $X=\gamma, e e, \mu \mu$ ) exhibit similar quadratic dependence on the small Yukawa parameter $a$. However, close to the largest perturbatively allowed values of $a$, i.e. $a \lesssim 0.34$ for the model (2.4) and $a \lesssim 0.23$ for the model (2.5), some of the observables of $\tau$-LFV exhibit either numerical instability, or the existence of a cancellation region in parameter space, as we will see below.

Figure 7 presents numerical estimates of $B(\mu \rightarrow e \gamma)$ [blue (solid) line], $B(\mu \rightarrow e e e)$ [red (dashed) line], $R_{\mu e}^{\mathrm{Ti}}$ [violet (dotted) line] and $R_{\mu e}^{\mathrm{Au}}$ [green (dash-dotted) line], as functions of $B(\mu \rightarrow e \gamma)$ (left pannels) and the heavy neutrino mass scale $m_{N}$ (right pannels). In all pannels, we keep the Yukawa parameter $a$ fixed by the condition $B(\mu \rightarrow e \gamma)=10^{-12}$ for $m_{N}=400 \mathrm{GeV}$, using the benchmark value: $\tan \beta=10$. The upper pannels display numerical values for the 

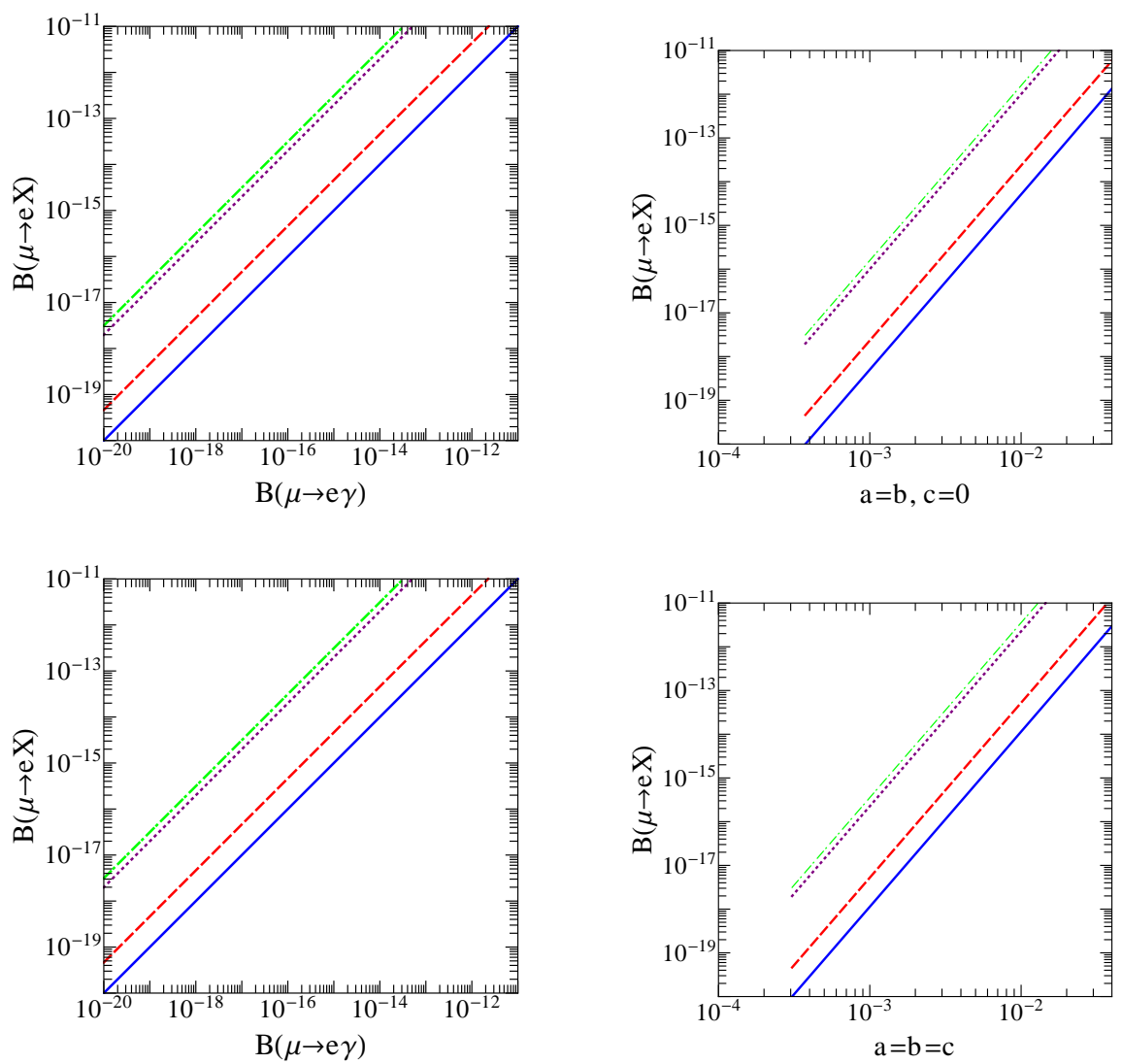

FIG. 4: The same as in Fig. 3. but for $m_{N}=1 \mathrm{TeV}$.

Yukawa texture (2.4), with $a=b$ and $c=0$, and the lower pannels for the Yukawa texture (2.5), with $a=b=c$. The heavy neutrino mass is varied within the LHC explorable range: $400 \mathrm{GeV}<m_{N}<10 \mathrm{TeV}$. All observables $B(\mu \rightarrow e X)$ of $\mu$-LFV (with $X=\gamma, e e, \mathrm{Ti}, \mathrm{Au}$ ) exhibit a non-trivial dependence on $m_{N}$. The branching ratio $B(\mu \rightarrow e \gamma)$ shows a dip at $m_{N} \approx 800 \mathrm{GeV}$ in both models (2.4) and (2.5), signifying the existence of a cancellation region in parameter space, due to loops involving heavy neutrino, sneutrino and soft SUSY-breaking terms. For $m_{N} \gtrsim 3 \mathrm{TeV}$, all observables tend to a constant value, as a result of the dominance of the soft SUSY-breaking contributions.

In Fig. 8 we show contours of the Yukawa parameters $(a, b, c)$ versus the heavy neutrino mass scale $m_{N}$, for $B(\mu \rightarrow e \gamma)$ [blue (solid) line], $B(\mu \rightarrow e e e)$ [red (dashed) line], $R_{\mu e}^{\mathrm{Ti}}$ [violet (dotted) line] and $R_{\mu e}^{\mathrm{Au}}$ [green (dash-dotted) line]. The Yukawa parameter $a$ and $m_{N}$ are determined by the condition $B(\mu \rightarrow e \gamma)=10^{-12}$. The labels in the vertical axes indicate the two Yukawa textures in (2.4) and (2.5), which we have adopted in our analysis. The contours for $B(\mu \rightarrow e \gamma)$ display a maximum for $m_{N} \approx 800 \mathrm{GeV}$, as a consequence of cancellations between heavy neutrino, sneutrino and soft SUSY-breaking contributions (cf. Fig. 7).

Figure 9 shows contours of the Yukawa parameters $(a, b, c)$, as functions of $m_{N}$, for $B(\tau \rightarrow e \gamma)$ [blue (solid) line], where the parameters $a$ and $m_{N}$ are determined by the condition $B(\tau \rightarrow e \gamma)=10^{-9}$. We do not give numerical results for $B(\tau \rightarrow \mu \gamma)$, as these are fully complementary to the ones given for $B(\tau \rightarrow e \gamma)$. Notice that given the above condition on $B(\tau \rightarrow e \gamma)$, no solution exists for $B(\tau \rightarrow e e e)$ and $B(\tau \rightarrow e \mu \mu)$.

In our numerical analysis so far, we have kept the value of $\tan \beta$ fixed to its benchmark value given in (4.1): $\tan \beta=10$. In Fig. 10, we relax this assumption, varying $\tan \beta$ in the interval $5 \lesssim \tan \beta \lesssim 20$, while maintaining agreement with a SM-like Higgs boson mass $M_{H} \approx 125 \mathrm{GeV}$ and taking into account that the combined experimental and theoretical errors are currently of the order of 5-6 GeV. Specifically, in Fig. 10 we display the dependence of $B(\mu \rightarrow e \gamma)$ [blue (solid) line], $B(\mu \rightarrow e e e)$ [red (dashed) line], $R_{\mu e}^{\mathrm{Ti}}$ [violet (dotted) line] and $R_{\mu e}^{\mathrm{Au}}$ [green (dash-dotted) line] on $\tan \beta$. In all pannels, the Yukawa parameter $a$ is determined by the condition $B(\mu \rightarrow e \gamma)=10^{-12}$. The upper pannels in Fig. 10 show numerical results for $m_{N}=400 \mathrm{GeV}$, while the lower pannels for $m_{N}=1 \mathrm{TeV}$. The left pannels give our predictions for the Yukawa texture (2.4), with $a=b$ and $c=0$, and the right pannels for the Yukawa texture (2.5), with $a=b=c$. In the lower pannels, we observe a suppression of $B(\mu \rightarrow e \gamma)$, for values $\tan \beta \approx 7$, due 

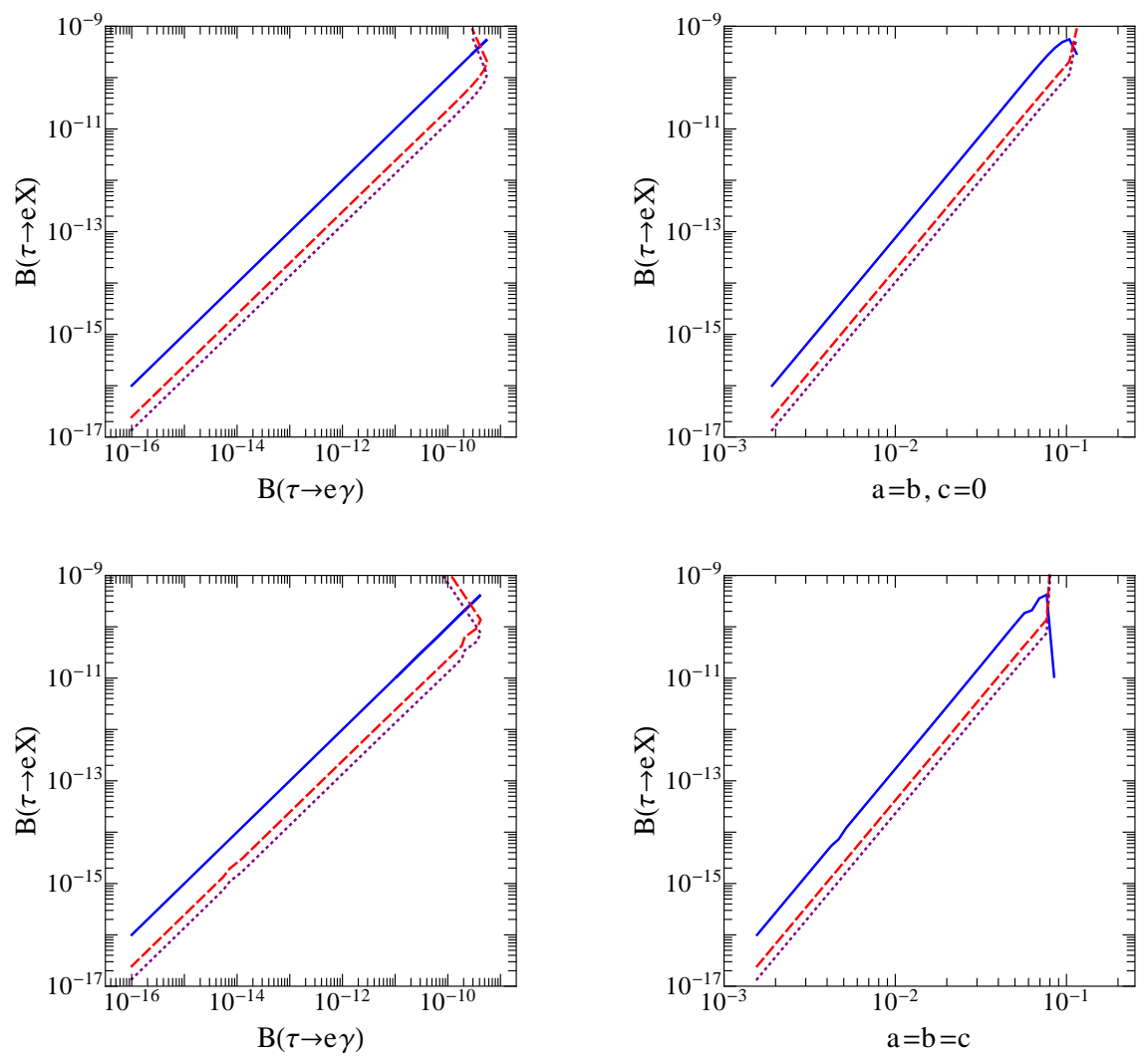

FIG. 5: Numerical estimates of $B(\tau \rightarrow e \gamma)$ [blue (solid)], $B(\tau \rightarrow e e e)$ [red (dashed)] and $B(\tau \rightarrow e \mu \mu)$ [violet (dotted)], as functions of $B(\tau \rightarrow e \gamma)$ (left pannels) and the Yukawa parameter $a$ (right pannels), for $m_{N}=400 \mathrm{GeV}$ and tan $\beta=10$. The upper pannels present predictions for the Yukawa texture (2.4), with $a=c$ and $b=0$, and the lower pannels for the Yukawa texture (2.5), with $a=b=c$.

to cancellation between heavy neutrino, sneutrino and soft SUSY-breaking effects.

It is interesting to compare the contributions of the magnetic dipole formfactors to the CLFV observables, with those originating from the remaining formfactors. Specifically, if one assumes that only the magnetic dipole formfactors $G_{\gamma}^{L, R}$ contribute in (3.10), (3.11) and (3.19), then the following analytical results are obtained for the ratios:

$$
\begin{aligned}
& R_{1} \equiv \frac{B\left(l \rightarrow l^{\prime} l_{1} l_{1}^{c}\right)}{B\left(l \rightarrow l^{\prime} \gamma\right)}=\frac{\alpha}{3 \pi}\left(\ln \frac{m_{l}^{2}}{m_{l^{\prime}}^{2}}-3\right) \\
& R_{2} \equiv \frac{B\left(l \rightarrow l^{\prime} l^{\prime} l^{\prime c}\right)}{B\left(l \rightarrow l^{\prime} \gamma\right)}=\frac{\alpha}{3 \pi}\left(\ln \frac{m_{l}^{2}}{m_{l^{\prime}}^{2}}-\frac{11}{4}\right) \\
& R_{3} \equiv \frac{R_{\mu e}^{J}}{B(\mu \rightarrow e \gamma)}=16 \alpha^{4} \frac{\Gamma_{\mu}}{\Gamma_{\text {capture }}} Z Z_{\text {eff }}^{4}\left|F\left(-\mu^{2}\right)\right|^{2} .
\end{aligned}
$$

According to the formulae (4.3)-(4.5), the predicted $R_{1}$ values for $\tau \rightarrow e \mu \mu$ and $\tau \rightarrow \mu e e$ are $1 / 90$ and $1 / 419$ respectively, the predicted $R_{2}$ values for $\mu \rightarrow e e e, \tau \rightarrow e e e$ and the $\tau \rightarrow \mu \mu \mu$ are 1/159,1/91 and 1/460 respectively, and the predicted $R_{3}$ values for Ti and $\mathrm{Au}$ are $1 / 198$ and $1 / 188$ respectively.

In Fig. 11 we give numerical estimates of the ratios $R_{2}(\mu \rightarrow e e e), R_{3}^{\mathrm{Ti}}$ and $R_{3}^{\mathrm{Au}}$, as functions of $m_{N}$. The Yukawa parameter $a$ is fixed by the condition $B(\mu \rightarrow e \gamma)=10^{-12}$, for $m_{N}=400 \mathrm{GeV}$ and $\tan \beta=10$. In the upper pannel, thick lines show the predicted values obtained by a complete evaluation of $R_{2}(\mu \rightarrow e e e)$ [blue (solid) line], $R_{3}^{\mathrm{Ti}}$ [red (dashed) line] and $R_{3}^{\mathrm{Au}}$ [violet (dotted) line], while the respective thin lines are obtained by keeping only the magnetic dipole formfactors $G_{\gamma}^{L}$ and $G_{\gamma}^{R}$. Hence, we see that going beyond the magnetic dipole moment approximation may enhance the ratios $R_{2,3}$ by more than two orders of magnitude.

The two middle pannels of Fig. 111 provide a formfactor analysis of $R_{2}(\mu \rightarrow e e e)$ and $R_{3}^{\mathrm{Au}}$, by considering separately the contributions due to $G_{\gamma}$ and $F_{\gamma}$ [blue (solid) line], $F_{Z}$ [red (dashed) line] and box formfactors [violet (dotted) line]. In particular, we observe that heavy neutrino contributions to the box formfactors become comparable to and even 

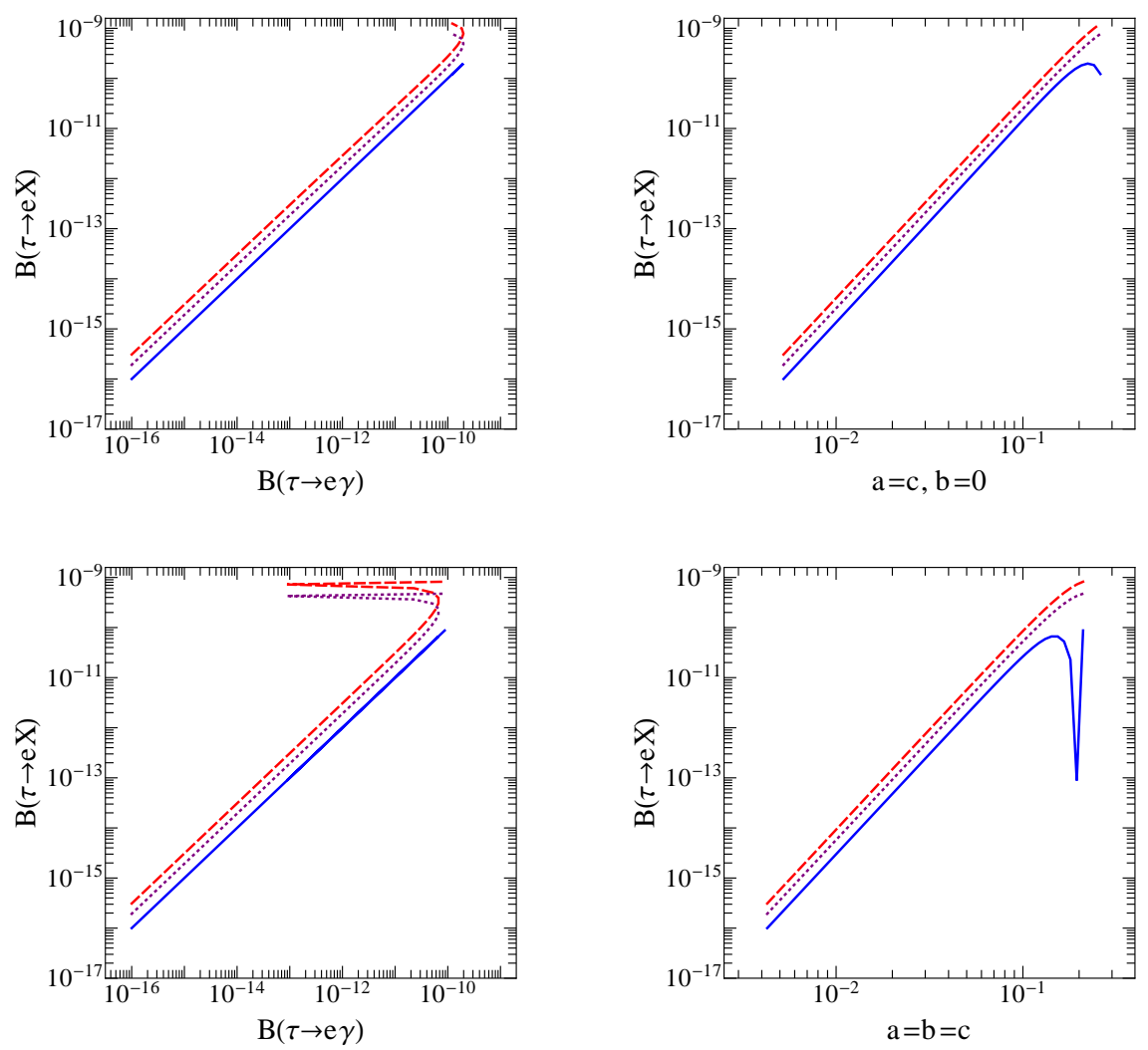

FIG. 6: The same as in Fig. 5] but for $m_{N}=1 \mathrm{TeV}$.

larger than the $Z$-boson mediated graphs in $\mu \rightarrow e$ conversion in Gold, for heavy neutrino masses $m_{N} \lesssim 1 \mathrm{TeV}$. We have checked that for $m_{N} \lesssim 1 \mathrm{TeV}$, box graphs due to heavy neutrinos also dominate the process of $\mu \rightarrow e$ conversion in Titanium (not explicitly shown in Fig. 11). Finally, the two lower pannels show the individual contributions due to heavy neutrinos $N_{1,2,3}$ [blue (solid) line], sneutrinos $\widetilde{N}_{1,2, \ldots, 12}$ [red (dashed) line] and soft SUSY-breaking LFV terms [violet (dotted) line]. From these two lower pannels, it is obvious that for heavy neutrino masses $m_{N} \gtrsim 1 \mathrm{TeV}$, the soft SUSY-breaking effects dominate the CLFV formfactors, which are tagged with the superscripts SB in Appendix C, Instead, for $m_{N} \lesssim 1 \mathrm{TeV}$, heavy-neutrino effects start becoming the leading contribution to the CLFV observables associated with the muon. Note that the green (horizontal) lines in the middle and lower pannels serve as reference values obtained by assuming that only the $G_{\gamma}^{L, R}$ formfactors contribute to the amplitudes.

An important consistency check for our numerical analysis has been to analytically show that all soft SUSY-breaking effects on the formfactors (C.4), (C.8) and (C.14) vanish in the limit of degenerate charged slepton masses. On the other hand, RG effects from $M_{\mathrm{GUT}}$ to $M_{Z}$ induce sizeable deviations to the charged slepton mass matrix from the unit matrix. As a consequence, unitarity cancellations due to the so-called GIM mechanism become less effective in this case and so render the SB part of the formfactors, such as $F_{l^{\prime} l Z}^{L, \mathrm{SB}}$ and $F_{l^{\prime} l Z}^{R, \mathrm{SB}}$, rather large.

Another essential check was to show that under the assumptions adopted in [44], our formfactor $F_{l^{\prime} l Z}^{L, \tilde{N}}$ given in (C.7) reduces to $\frac{2 c_{W}}{g} F_{L}^{c}$, where $F_{L}^{c}$ is one of the formfactors defined in (6) of [44], which in turn can be shown to vanish. The assumptions in [44] are: (i) the standard seesaw mechanism with ultra-heavy right neutrinos, (ii) no charged wino or higgsino mixing, and (iii) the dominance of the wino contribution. Under these three assumptions, the interaction vertices occurring in the formfactor $F_{l^{\prime} l Z}^{L, \tilde{N}}$ simplify as follows:

$$
\tilde{B}_{l m A}^{R, 1}, \tilde{B}_{l m A}^{R, 2} \rightarrow-U_{l k}, \quad \tilde{C}_{A B}^{1}, \tilde{C}_{A B}^{2}, \tilde{C}_{A B}^{3}, \tilde{C}_{A B}^{4} \rightarrow-\frac{1}{2} \delta_{k k^{\prime}}, \quad V_{m k}^{\tilde{\chi}^{-} R} \rightarrow c_{w}^{2},
$$

where $A, B$ assume now the restricted range of values $k, k^{\prime}=1,2,3$ and $U$ is a $3 \times 3$ unitary matrix. Given the simplifications in (4.6), we recover the expression of [44], resulting in the replacement: $F_{l^{\prime} l Z}^{L, \tilde{N}} \rightarrow \frac{2 c_{W}}{g} F_{L}^{c}$. The above non-trivial checks provide firm support for the correctness of our analytical and numerical results that we have presented in this section. 

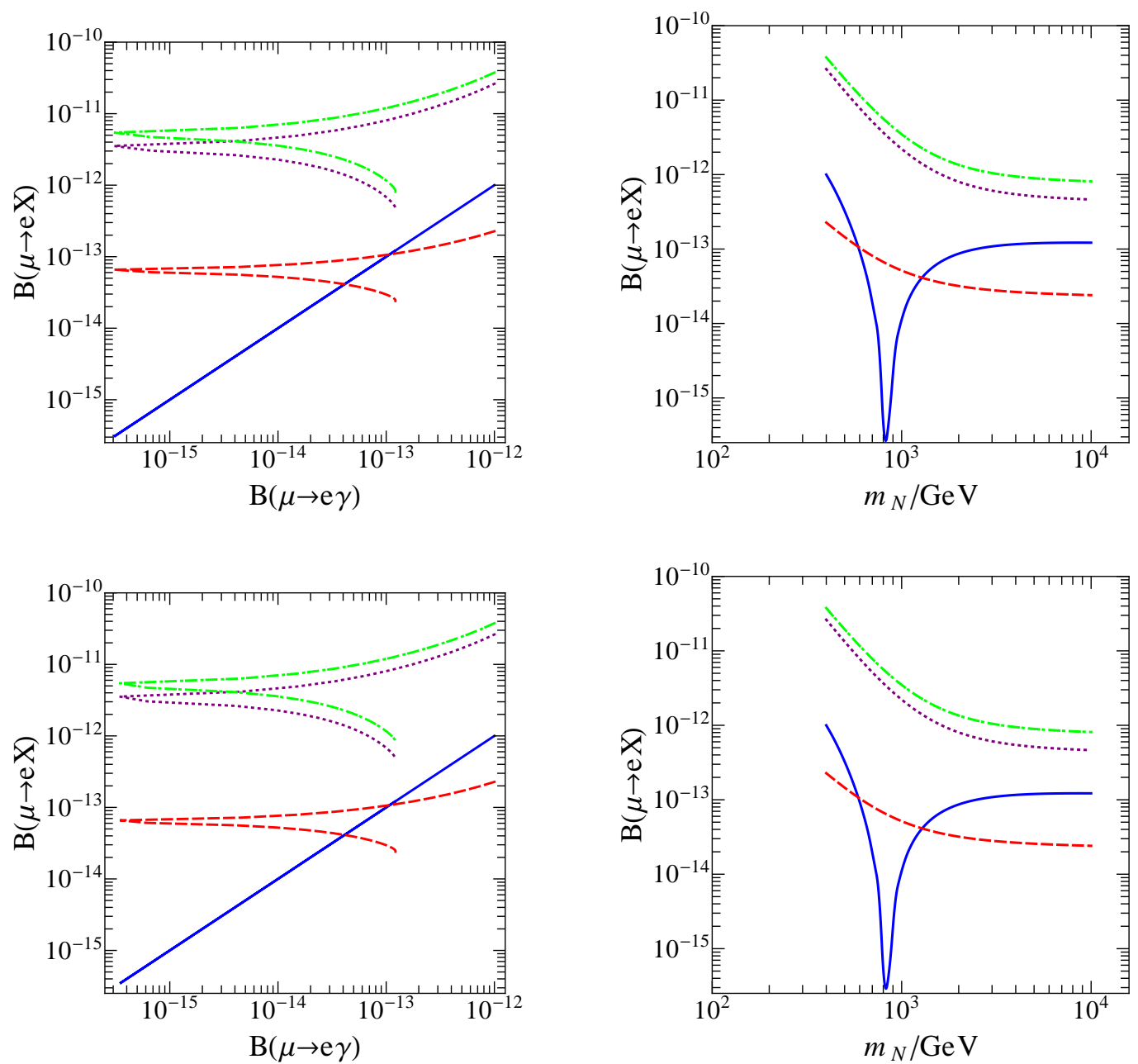

FIG. 7: Numerical estimates of $B(\mu \rightarrow e \gamma)$ [blue (solid)], $B(\mu \rightarrow e e e)$ [red (dashed)], $R_{\mu e}^{\mathrm{Ti}}$ [violet (dotted)] and $R_{\mu e}^{\mathrm{Au}}$ [green (dash-dotted)], as functions of $B(\mu \rightarrow e \gamma)$ (left pannels) and the heavy neutrino mass scale $m_{N}$ (right pannels). In all pannels, the Yukawa parameter $a$ was kept fixed by the condition $B(\mu \rightarrow e \gamma)=10^{-12}$ for $m_{N}=400 \mathrm{GeV}$, and tan $\beta=10$ was used. The upper pannels display numerical values for the Yukawa texture (2.4), with $a=b$ and $c=0$, and the lower pannels for the Yukawa texture (2.5), with $a=b=c$.
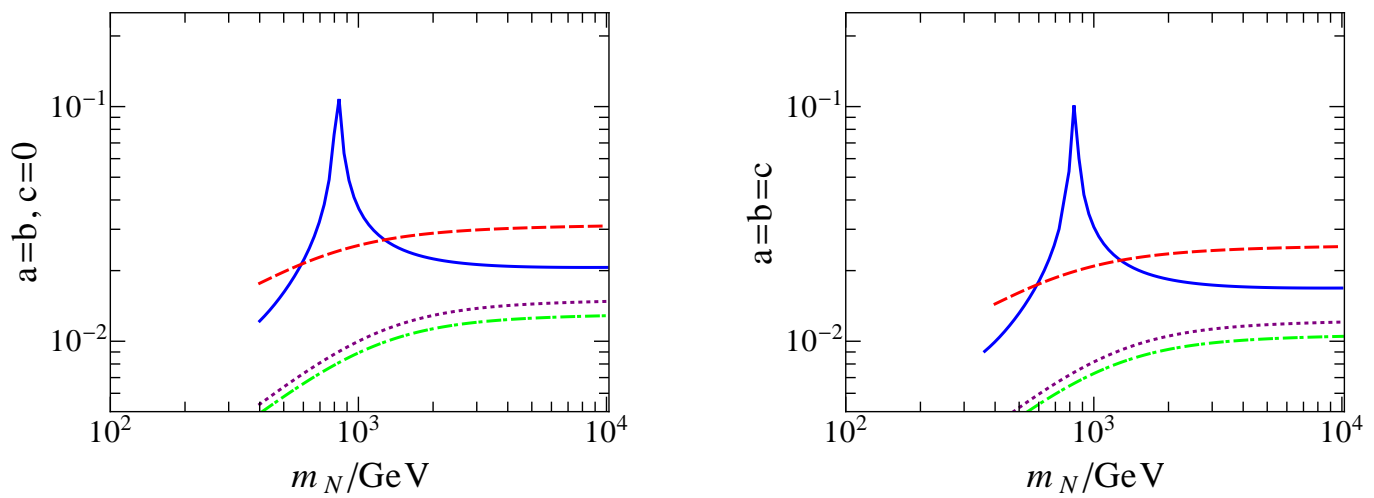

FIG. 8: Contours of the Yukawa parameters $(a, b, c)$ versus $m_{N}$, for $B(\mu \rightarrow e \gamma)$ [blue (solid)], $B(\mu \rightarrow e e e)$ [red (dashed)], $R_{\mu e}^{\mathrm{Ti}}$ [violet (dotted)] and $R_{\mu e}^{\mathrm{Au}}$ [green (dash-dotted)], where $a$ and $m_{N}$ are determined by the condition $B(\mu \rightarrow e \gamma)=10^{-12}$. All contours are evaluated with $\tan \beta=10$ and for different Yukawa textures, as indicated by the vertical axes labels. 

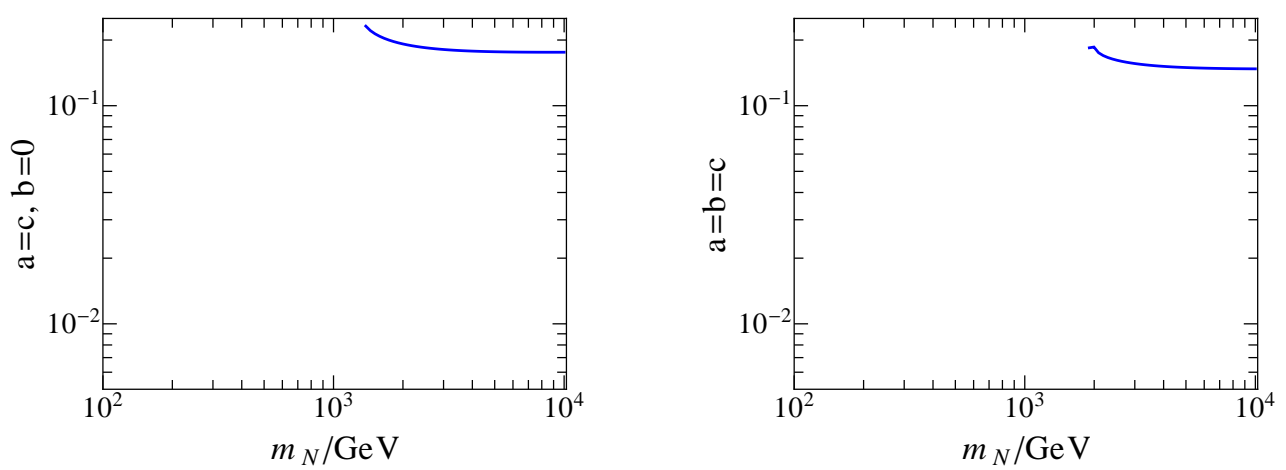

FIG. 9: Contours of the Yukawa parameters $(a, b, c)$ versus $m_{N}$, for $B(\tau \rightarrow e \gamma)$ [blue (solid)], where tan $\beta=10$ and $a$ and $m_{N}$ are determined by the condition $B(\tau \rightarrow e \gamma)=10^{-9}$. No solutions have been found for $B(\tau \rightarrow e e e)$ and $B(\tau \rightarrow e \mu \mu)$.
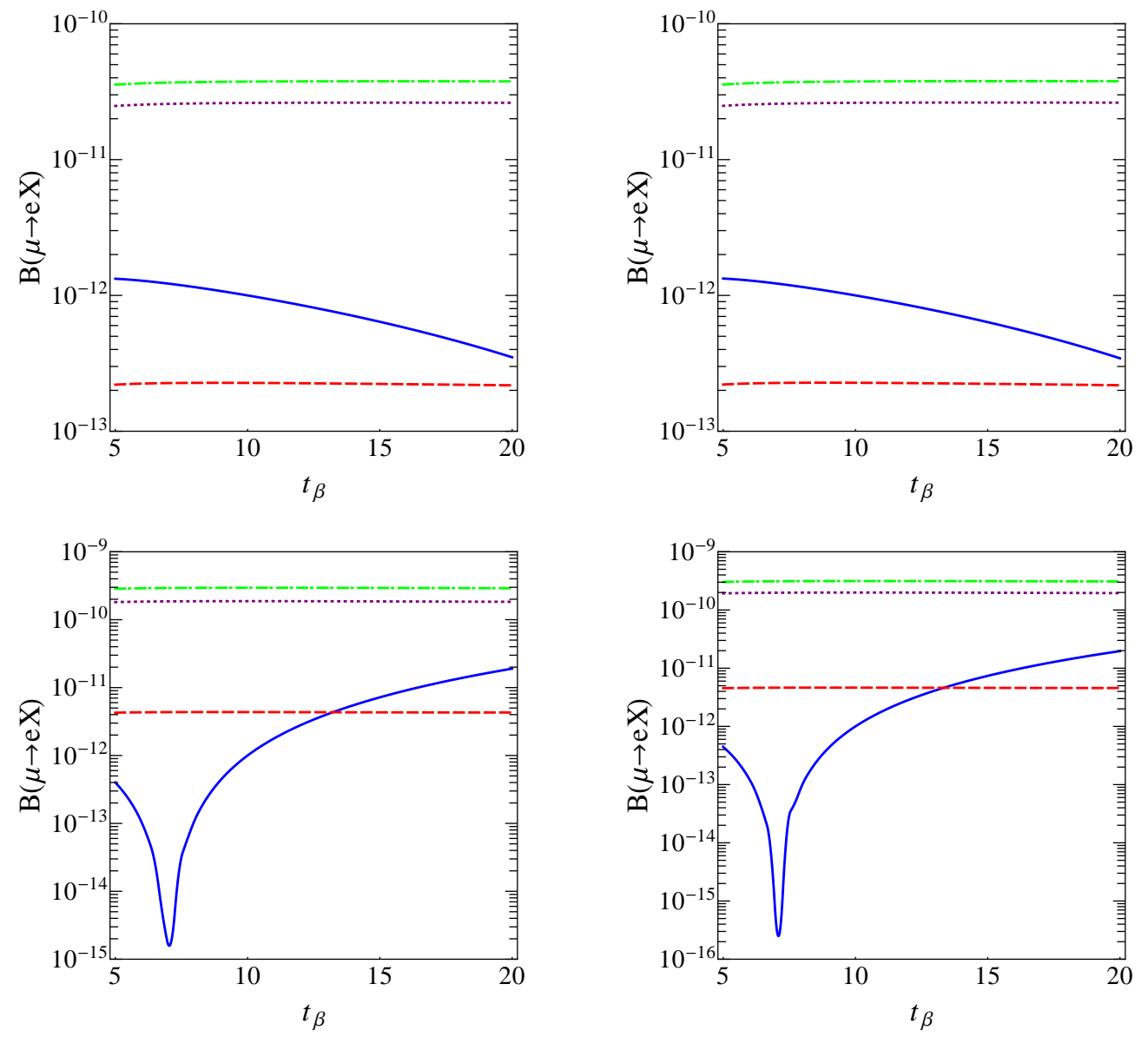

FIG. 10: Numerical estimates of $B(\mu \rightarrow e \gamma)$ [blue (solid)], $B(\mu \rightarrow e e e)$ [red (dashed)], $R_{\mu e}^{\mathrm{Ti}}$ [violet (dotted)] and $R_{\mu e}^{\mathrm{Au}}[\mathrm{green}$ (dash-dotted)], as functions of $\tan \beta$. The upper pannels are obtained for $m_{N}=400 \mathrm{GeV}$ and the lower pannels for $m_{N}=1 \mathrm{TeV}$. The left pannels use the Yukawa texture (2.4), with $a=b$ and $c=0$, and the right pannels the Yukawa texture (2.5), with $a=b=c$. In all pannels, the Yukawa parameter $a$ is determined by the condition $B(\mu \rightarrow e \gamma)=10^{-12}$.

\section{CONCLUSIONS}

We have analyzed Charged Lepton Flavour Violation in the MSSM extended by low-scale singlet heavy neutrinos, paying special attention to the individual loop contributions due to the heavy neutrinos $N_{1,2,3}$, sneutrinos $\widetilde{N}_{1,2, \ldots, 12}$ and soft SUSY-breaking terms. In our analysis, we have included for the first time the complete set of box diagrams, 

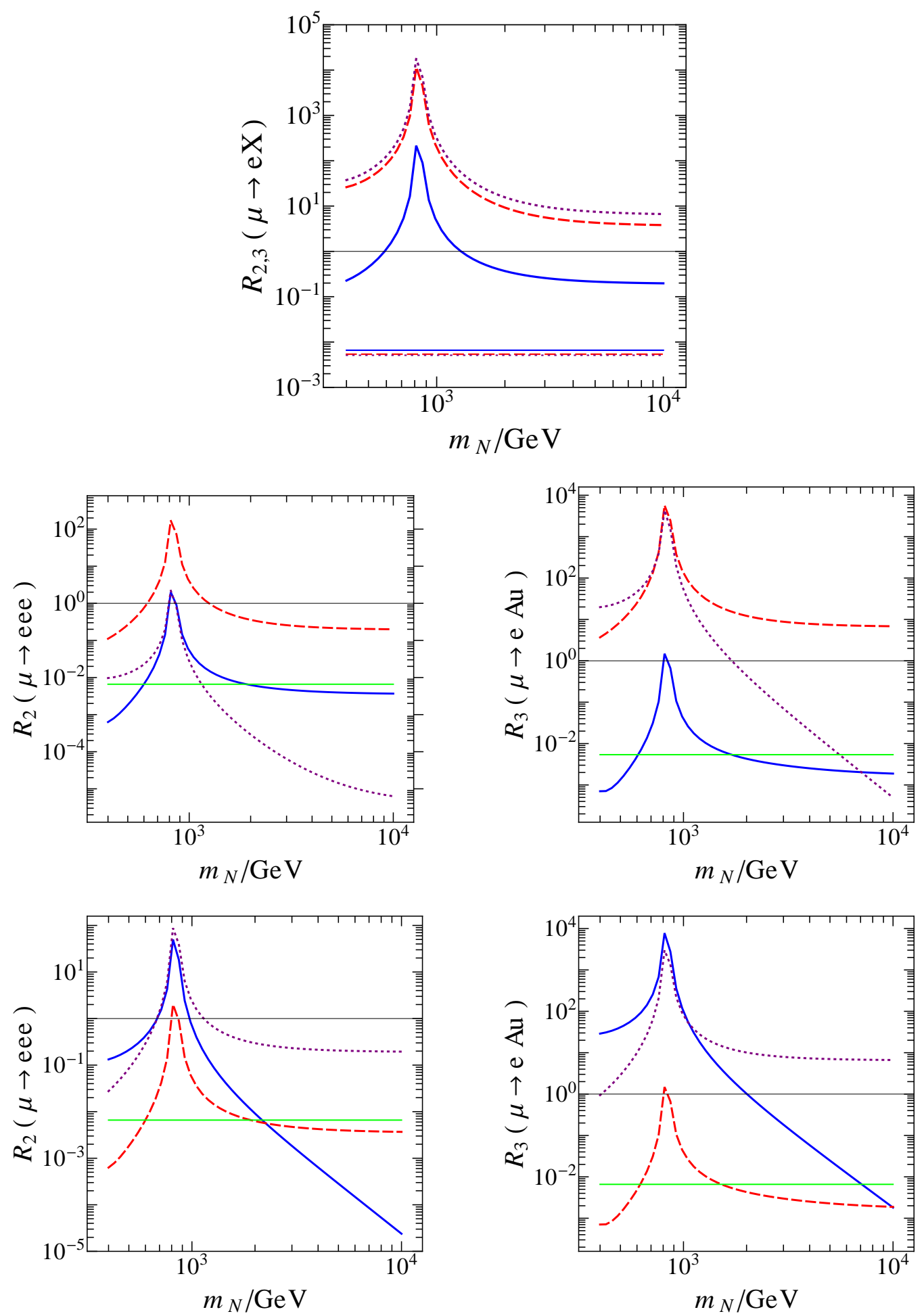

FIG. 11: Numerical estimates of the ratios $R_{2}(\mu \rightarrow$ eee $), R_{3}^{\mathrm{Ti}}$ and $R_{3}^{\mathrm{Au}}$, as functions of $m_{N}$. The Yukawa parameter $a$ is fixed by the condition $B(\mu \rightarrow e \gamma)=10^{-12}$, for $m_{N}=400 \mathrm{GeV}$ and $\tan \beta=10$. In the upper pannel, thick lines give the complete evaluation of $R_{2}\left(\mu \rightarrow\right.$ eee) [blue (solid)], $R_{3}^{\mathrm{Ti}}$ [red (dashed)] and $R_{3}^{\mathrm{Au}}$ [violet (dotted)], while the respective thin lines are evaluated keeping only the magnetic dipole formfactors $G_{\gamma}^{L}$ and $G_{\gamma}^{R}$. The two middle pannels provide a formfactor analysis of $R_{2}(\mu \rightarrow e e e)$ and $R_{3}^{\mathrm{Au}}$, in terms of contributions due to $G_{\gamma}$ and $F_{\gamma}$ [blue (solid)], $F_{Z}$ [red (dashed)] and box formfactors [violet (dotted)]. The lower two pannels show the separate contributions due to heavy neutrinos $N$ [blue (solid)], sneutrinos $\widetilde{N}$ [red (dashed)] and soft SUSY-breaking LFV terms [violet (dotted)]. The green (horizontal) lines in the middle and lower pannels give the predicted values obtained by assuming that only the $G_{\gamma}^{L, R}$ formfactors contribute to the amplitudes. 
in addition to the photon and the $Z$-boson mediated interactions. We have also derived the complete set of chiral amplitudes and their associate formfactors related to the neutrinoless three-body CLFV decays of the muon and tau, such as $\mu \rightarrow e e e, \tau \rightarrow \mu \mu \mu, \tau \rightarrow e \mu \mu$ and $\tau \rightarrow e e \mu$, and to the coherent $\mu \rightarrow e$ conversion in nuclei. Our analytical results are general and can be applied to most of the New Physics models with CLFV. In this context, we emphasize that our systematic analysis has revealed the existence of two new box formfactors, which have not been considered before in the existing literature of New Physics theories with CLFV.

Our detailed study has shown that the soft SUSY-breaking effects in the $Z$-boson-mediated graphs dominate the CLFV observables, for appreciable regions of the $\nu_{R}$ MSSM parameter space in mSUGRA. Nevertheless, there is a significant portion of parameter space for heavy neutrino masses $m_{N} \lesssim 1 \mathrm{TeV}$, where box diagrams involving heavy neutrinos in the loop can be comparable to, or even larger than the corresponding $Z$-boson-exchange diagrams in $\mu \rightarrow e e e$ and in $\mu \rightarrow e$ conversion in nuclei (cf. Fig. [11). In the same kinematic regime, due to accidental cancellations, we have also observed a suppression of the branching ratios for the photonic CLFV decays $\mu \rightarrow e \gamma$, as well as for the decays $\tau \rightarrow e \gamma$ and $\tau \rightarrow \mu \gamma$. As mentioned in the Introduction, such a suppression in low-scale seesaw models is a consequence of a cancellation between particle and sparticle contributions due to the approximate realization of the SUSY no-go theorem due to Ferrara and Remiddi [43]. Instead, in high-scale seesaw models such cancellations can only occur for particular choices of the neutrino-Yukawa and Majorana-mass textures [39, 52]. Hence, the results obtained within supersymmetric low-scale seesaw type-I models, with $m_{N} \lesssim 10 \mathrm{TeV}$, corroborate the original findings in 34], where the usual paradigm with the photon dipole-moment operators dominating the CLFV observables in high-scale seesaw models [37, 38] gets radically modified, such that $\mu \rightarrow e e e$ and $\mu \rightarrow e$ conversion may also represent sensitive probes of CLFV.

We have found that unlike heavy neutrinos, CLFV effects induced by sneutrinos remain subdominant for the entire region of the mSUGRA parameter space. In addition, the perturbativity constraint on the neutrino Yukawa couplings $\mathbf{h}_{\nu}$ up to the GUT scale renders the quartic coupling contributions of order $\left(\mathbf{h}_{\nu}\right)^{4}$ small. The present study has focused on providing numerical predictions for relatively small and intermediate values of $\tan \beta$, i.e. $\tan \beta \lesssim 20$, where neutral Higgs-mediated interactions constrained by the recent LHCb observation of the decay $B_{s} \rightarrow \mu \mu$ are not expected to give sizeable contributions. A global analysis that includes large $\tan \beta$ effects on CLFV observables and LHC constraints will be given in a forthcoming communication.

\section{Acknowledgements}

We thank George Lafferty for valuable discussions regarding experimental sensitivities at the intensity frontier of CLFV. The work of AP is supported in part by the Lancaster-Manchester-Sheffield Consortium for Fundamental Physics under STFC grant ST/J000418/1. AP also acknowledges partial support by an IPPP associateship from Durham University. The work of AI and LP was supported by the Ministry of Science, Sports and Technology under contract 119-0982930-1016. 


\section{Appendix A: INTERACTION VERTICES}

In this appendix, we list the Lagrangians describing the interaction vertices required to calculate the transition amplitudes for the CLFV processes under study. The corresponding interaction vertices for the SM and the MSSM are obtained by adopting the conventions of the public code FeynArts-3.3. FVMSSM. mod. The Lagrangians of interest to us include:

1. Vertices from 2HDM sector of the MSSM involving SM particles only,

$$
\mathcal{L}_{\bar{d} u H^{-}}+\text {H.c. }=\frac{g_{w}}{\sqrt{2} M_{W}} V_{i j}^{*} \bar{d}_{j}\left(t_{\beta} m_{d_{j}} P_{L}+t_{\beta}^{-1} m_{u_{i}} P_{R}\right) u_{i} H^{-}+\text {H.c. }
$$

Here $H^{-}$is the negatively charged Higgs scalar, $V$ is the Cabibbo-Kobayashi-Maskawa matrix, $m_{d_{i}}$ and $m_{u_{i}}$ are the quark masses and $c_{w}=\cos \theta_{w}$.

2. Vertices of singlet neutrinos in the $\nu_{R} \mathrm{SM}$ sector of the MSSM,

$$
\begin{aligned}
\mathcal{L}_{\bar{e} n G^{-}}+\text {H.c. } & =\frac{g_{w}}{\sqrt{2} M_{W}} B_{i a} \bar{e}_{i}\left(-m_{e_{i}} P_{L}+m_{n_{a}} P_{R}\right) n_{a} G^{-}+\text {H.c. }, \\
\mathcal{L}_{\bar{e} n W^{-}}+\text {H.c. } & =-\frac{g_{w}}{\sqrt{2}} B_{i a} \bar{e}_{i} \gamma^{\mu} P_{L} n_{a} W_{\mu}^{-}+\text {H.c. }, \\
\mathcal{L}_{\bar{n} n Z} & =-\frac{g_{w}}{2 c_{W}} C_{a b} \bar{n}_{a} \gamma^{\mu} P_{L} n_{b} Z_{\mu} .
\end{aligned}
$$

Here $n_{a}$ and $m_{n_{a}}$ denote the neutrino mass-eigenstates and their respective masses and $B$ and $C$ are lepton flavour mixing matrices defined in [29] and [33]. The matrices $B$ and $C$ satisfy the following set of relations:

$$
\begin{aligned}
B_{l a} B_{l^{\prime} a}^{*} & =\delta_{l l^{\prime}}, \quad C_{a c} C_{b c}=C_{a b}, \quad B_{l b} C_{b a}=B_{l a}, \quad B_{l a}^{*} B_{l b}=C_{a b}, \\
m_{a} C_{a c} C_{b c} & =0, \quad m_{a} B_{l b} C_{b a}^{*}=0, \quad m_{a} B_{l a} B_{l^{\prime} a}=0 .
\end{aligned}
$$

3. Vertices from the 2HDM sector of the MSSM involving Majorana neutrinos,

$$
\mathcal{L}_{\bar{e} n H^{-}}+\text {H.c. }=\frac{g_{w}}{\sqrt{2} M_{W}} B_{i a} \bar{e}_{i}\left(t_{\beta} m_{e_{i}} P_{L}+t_{\beta}^{-1} m_{n_{a}} P_{R}\right) n_{a} H^{-}+\text {H.c. }
$$

4. MSSM vertices with sparticles,

$$
\begin{aligned}
& \mathcal{L}_{\bar{d} \tilde{\chi}^{-} \tilde{u}}+\text { H.c. }=g_{w} \bar{d}_{j}\left(\tilde{V}_{j m a}^{-d L} P_{L}+\tilde{V}_{j m a}^{-d R} P_{R}\right) \tilde{\chi}_{m}^{-} \tilde{u}_{a}+\text { H.c. }, \\
& \mathcal{L}_{\bar{u} \tilde{\chi}^{+} \tilde{d}}+\text { H.c. }=g_{w} \bar{u}_{j}\left(\tilde{V}_{j m a}^{+u L} P_{L}+\tilde{V}_{j m a}^{+u L} P_{R}\right) \tilde{\chi}_{m}^{+} \tilde{d}_{a}+\text { H.c. }, \\
& \mathcal{L}{\overline{\tilde{\chi}^{-}}}_{\tilde{\chi}^{-} A}+\mathcal{L}{\overline{\tilde{\chi}^{+}}}_{\tilde{\chi}^{+} A}=e \overline{\tilde{\chi}_{m}^{-}} \gamma^{\mu} \tilde{\chi}_{m}^{-} A_{\mu}-e \overline{\tilde{\chi}_{m}^{+}} \gamma^{\mu} \tilde{\chi}_{m}^{+} A_{\mu}, \\
& \mathcal{L}_{\tilde{\tilde{\chi}}^{-} \tilde{\chi}^{-} Z}+\mathcal{L}_{\tilde{\chi}^{+} \tilde{\chi}^{+} Z}=\frac{g_{w}}{c_{W}} \overline{\tilde{\chi}_{m}^{-}} \gamma^{\mu}\left(V_{m k}^{\tilde{\chi}^{-} L} P_{L}+V_{m k}^{\tilde{\chi}^{-} R} P_{R}\right) \tilde{\chi}_{k}^{-} Z_{\mu} \\
& -\frac{g_{w}}{c_{W}} \overline{\tilde{\chi}_{m}^{+}} \gamma^{\mu}\left(V_{m k}^{\tilde{\chi}^{-} L *} P_{R}+V_{m k}^{\tilde{\chi}^{-} R *} P_{L}\right) \tilde{\chi}_{k}^{+} Z_{\mu}, \\
& \mathcal{L}_{\overline{\tilde{\chi}}^{0} \tilde{\chi}^{0} Z}=\frac{g}{c_{w}} \overline{\tilde{\chi}}_{m}^{0}\left(\gamma^{\mu} P_{L} V_{m k}^{\tilde{\chi}^{0} L}+\gamma^{\mu} P_{R} V_{m k}^{\tilde{\chi}^{0} R}\right) \tilde{\chi}_{k}^{0} Z_{\mu} \\
& \mathcal{L}_{\tilde{e}^{*} \tilde{e} Z}=g_{w} \tilde{V}_{a b}^{\tilde{e}} \tilde{e}_{a}^{*} i \stackrel{\leftrightarrow}{\partial} \tilde{e}^{\mu} Z_{\mu} \\
& \mathcal{L}_{\bar{e} \tilde{\chi}^{0} \tilde{e}}+\mathcal{L}_{\bar{\chi}^{0} e \tilde{e}^{*}}=g_{w} \bar{e}_{j}\left(P_{L} \tilde{V}_{j m a}^{0 e L}+P_{R} \tilde{V}_{j m a}^{0 e R}\right) \tilde{\chi}^{0} \tilde{e}_{a}+\text { H.c. }, \\
& \mathcal{L}_{\bar{u} \tilde{\chi}^{0} \tilde{u}}+\mathcal{L}_{\bar{\chi}^{0} u \tilde{u}^{*}}=g_{w} \bar{u}_{j}\left(P_{L} \tilde{V}_{j m a}^{0 u L}+P_{R} \tilde{V}_{j m a}^{0 u R}\right) \tilde{\chi}^{0} \tilde{u}_{a}+\text { H.c. }, \\
& \mathcal{L}_{\bar{d} \tilde{\chi}^{0} \tilde{d}}+\mathcal{L}_{\tilde{\chi}^{0} d \tilde{d}^{*}}=g_{w} \bar{d}_{j}\left(P_{L} \tilde{V}_{j m a}^{0 d L}+P_{R} \tilde{V}_{j m a}^{0 d R}\right) \tilde{\chi}^{0} \tilde{d}_{a}+\text { H.c. },
\end{aligned}
$$

where

$$
\tilde{V}_{j m a}^{-d L}=\frac{m_{d_{j}}}{\sqrt{2} c_{\beta} M_{W}} \mathcal{U}_{m 2}^{*} V_{i j}^{*}\left(R_{L}^{\tilde{u}}\right)_{a i}^{*}
$$




$$
\begin{aligned}
\tilde{V}_{j m a}^{-d R} & =-\mathcal{V}_{m 1} V_{i j}^{*}\left(R_{L}^{\tilde{u}}\right)_{a i}^{*}+\frac{m_{u_{i}}}{\sqrt{2} s_{\beta} M_{W}} \mathcal{V}_{m 2} V_{i j}^{*}\left(R_{R}^{\tilde{u}}\right)_{a i}^{*}, \\
\tilde{V}_{j m a}^{+u L} & =\frac{m_{u_{j}}}{\sqrt{2} s_{\beta} M_{W}} \mathcal{V}_{m 2}^{*} V_{j i}\left(R_{L}^{\tilde{d}}\right)_{a i}^{*}, \\
\tilde{V}_{j m a}^{+u R} & =-\mathcal{U}_{m 1} V_{j i}\left(R_{L}^{\tilde{d}}\right)_{a i}^{*}+\frac{m_{d_{i}}}{\sqrt{2} c_{\beta} M_{W}} \mathcal{U}_{m 2} V_{j i}\left(R_{R}^{\tilde{d}}\right)_{a i}^{*}, \\
V_{m k}^{\tilde{\chi}^{-} L} & =\mathcal{U}_{m 1} \mathcal{U}_{k 1}^{*}+\frac{1}{2} \mathcal{U}_{m 2} \mathcal{U}_{k 2}^{*}-\delta_{m k} s_{w}^{2}, \\
V_{m k}^{\tilde{\chi}^{-} R} & =\mathcal{V}_{m 1}^{*} \mathcal{V}_{k 1}+\frac{1}{2} \mathcal{V}_{m 2}^{*} \mathcal{V}_{k 2}-\delta_{m k} s_{w}^{2} \\
V_{m k}^{\tilde{\chi}^{0} L} & =-\frac{1}{4}\left(Z_{m 3} Z_{k 3}^{*}-Z_{m 4} Z_{k 4}^{*}\right), \\
V_{m k}^{\tilde{\chi}^{0} R} & =\frac{1}{4}\left(Z_{m 3}^{*} Z_{k 3}-Z_{m 4}^{*} Z_{k 4}\right) \\
\tilde{V}_{a b}^{\tilde{e}} & =\frac{c_{2 w}}{c_{w}}\left(R_{L}^{\tilde{e}}\right)_{a i}\left(R_{L}^{\tilde{e}}\right)_{b i}^{*}-\frac{s_{w}^{2}}{c_{w}}\left(R_{R}^{\tilde{e}}\right)_{a i}\left(R_{R}^{\tilde{e}}\right)_{b i}^{*}, \\
\tilde{V}_{j m a}^{0 \ell L} & =-\sqrt{2} t_{w} Z_{m 1}^{*}\left(R_{R}^{\tilde{e}}\right)_{a j}^{*}-\frac{\left(m_{e}\right)_{j}}{\sqrt{2} c_{\beta} M_{W}} Z_{m 3}^{*}\left(R_{L}^{\tilde{e}}\right)_{a j}^{*}, \\
\tilde{V}_{j m a}^{0 \ell R} & =\frac{1}{\sqrt{2} c_{W}}\left(c_{W} Z_{m 2}+s_{W} Z_{m 1}\right)\left(R_{L}^{\tilde{e}}\right)_{a j}^{*}-\frac{\left(m_{e}\right)_{j}}{\sqrt{2} c_{\beta} M_{W}} Z_{m 3}\left(R_{R}^{\tilde{e}}\right)_{a j}^{*}, \\
\tilde{V}_{j m a}^{0 u L} & =\frac{2 \sqrt{2}}{3} t_{w} Z_{m 1}^{*}\left(R_{R}^{\tilde{u}}\right)_{a j}^{*}-\frac{\left(m_{u}\right)_{j}}{\sqrt{2} s_{\beta} M_{W}} Z_{m 4}^{*}\left(R_{L}^{\tilde{u}}\right)_{a j}^{*}, \\
\tilde{V}_{j m a}^{0 u R} & =-\frac{1}{2 c_{W}}\left(c_{W} Z_{m 2}+\frac{1}{3} s_{W} Z_{m 1}\right)\left(R_{L}^{\tilde{u}}\right)_{a j}^{*}-\frac{\left(m_{u}\right)_{j}}{\sqrt{2} s_{\beta} M_{W}} Z_{m 4}\left(R_{R}^{\tilde{u}}\right)_{a j}^{*}, \\
\tilde{V}_{j m a}^{0 d L} & =-\frac{\sqrt{2}}{3} t_{w} Z_{m 1}^{*}\left(R_{R}^{\tilde{d}}\right)_{a j}^{*}-\frac{\left(m_{d}\right)_{j}}{\sqrt{2} c_{\beta} M_{W}} Z_{m 3}\left(R_{L}^{\tilde{d}}\right)_{a j}^{*}, \\
\tilde{V}_{j m a}^{0 d R} & =\frac{1}{2 c_{W}}\left(c_{W} Z_{m 2}-\frac{1}{3} s_{W} Z_{m 1}\right)\left(R_{L}^{\tilde{d}}\right)_{a j}^{*}-\frac{\left(m_{d}\right)_{j}}{\sqrt{2} c_{\beta} M_{W}} Z_{m 3}\left(R_{R}^{\tilde{d}}\right)_{a j}^{*},
\end{aligned}
$$

and $c_{2 w}=\cos 2 \theta_{w}$. The unitary matrices diagonalizing the chargino mass matrix $\mathcal{U}$ and $\mathcal{V}$ and the unitary matrix diagonalizing the neutralino mass matrix $Z$ are taken from [69]. The matrices

$$
R_{a k}^{\tilde{f} L} \equiv U_{i a}^{\tilde{f}} U_{i k}^{f_{L} *}, \quad R_{a k}^{\tilde{f} R} \equiv U_{i+3 a}^{\tilde{f}} U_{i k}^{f_{R} *},
$$

with $f=d, u, e, a=1,2, \ldots, 6$ and $i, k=1,2,3$, quantify the disalignment between fermions and sfermions. Here $U^{f_{L}}, U^{f_{R}}$ and $U^{\tilde{f}}$ are unitary matrices that diagonalize the fermion and sfermion mass matrices, respectively.

5. Sneutrino vertices in the $\nu_{R} \mathrm{MSSM}$,

$$
\begin{aligned}
\mathcal{L}_{\bar{e} \tilde{\chi}^{-} \tilde{N}}+\text { H.c. } & =g_{w} \tilde{N}_{A} \bar{\ell}_{l}\left(P_{L} \frac{m_{l}}{\sqrt{2} c_{\beta} M_{W}} \tilde{B}_{l m A}^{L, 1}+P_{R} \tilde{B}_{l m A}^{R, 1}\right) \tilde{\chi}_{m}^{-}+\text {H.c. } \\
& =g_{w} \tilde{N}_{A}^{*} \bar{\ell}_{l}\left(P_{L} \frac{m_{l}}{\sqrt{2} c_{\beta} M_{W}} \tilde{B}_{l m A}^{L, 2}+P_{R} \tilde{B}_{l m A}^{R, 2}\right) \tilde{\chi}_{m}^{-}+\text {H.c. } \\
\mathcal{L}_{\tilde{N} \tilde{N} Z} & =\frac{g_{w}}{c_{W}} \tilde{C}_{A B}^{1} \tilde{N}_{A}^{*} i \stackrel{\leftrightarrow}{\partial} \tilde{N}_{B} Z_{\mu} \\
& =\frac{g_{w}}{c_{W}} \tilde{C}_{A B}^{2} \tilde{N}_{A}^{*} i \stackrel{\leftrightarrow}{\partial}^{\mu} \tilde{N}_{B}^{*} Z_{\mu} \\
& =\frac{g_{w}}{c_{W}} \tilde{C}_{A B}^{3} \tilde{N}_{A} i \stackrel{\leftrightarrow}{\partial}^{\mu} \tilde{N}_{B} Z_{\mu} \\
& =\frac{g_{w}}{c_{W}} \tilde{C}_{A B}^{4} \tilde{N}_{A} i \stackrel{\leftrightarrow}{\partial} \tilde{N}_{B}^{*} Z_{\mu}
\end{aligned}
$$

where

$$
\tilde{B}_{l m A}^{L, 1}=\mathcal{U}_{m 2} U_{i l}^{\ell_{R} *} \mathcal{U}_{i A}^{\tilde{\nu}},
$$




$$
\begin{aligned}
\tilde{B}_{l m A}^{L, 2} & =\mathcal{U}_{m 2} U_{i l}^{\ell_{R} *} \mathcal{U}_{i+6 A}^{\tilde{\nu} *}, \\
\tilde{B}_{l m A}^{R, 1} & =-\mathcal{U}_{i A}^{\tilde{\nu}} U_{i l}^{\ell_{L} *} \mathcal{V}_{m 1}+\frac{m_{n_{a}}}{\sqrt{2} s_{\beta} M_{W}} \mathcal{V}_{m 2} \mathcal{U}_{i+9 A}^{\tilde{\nu}} U_{i+3 a}^{\nu *} B_{l a} \\
\tilde{B}_{l m A}^{R, 2} & =-\mathcal{U}_{i+6 A}^{\tilde{\nu} *} U_{i l}^{\ell_{L} *} \mathcal{V}_{m 1}+\frac{m_{n_{a}}}{\sqrt{2} s_{\beta} M_{W}} \mathcal{V}_{m 2} \mathcal{U}_{i+3 A}^{\tilde{\nu} *} U_{i+3 a}^{\nu *} B_{l a}, \\
\tilde{C}_{A B}^{1} & =-\frac{1}{2} \mathcal{U}_{i A}^{\tilde{\nu} *} \mathcal{U}_{i B}^{\tilde{\nu}}, \\
\tilde{C}_{A B}^{2} & =-\frac{1}{2} \mathcal{U}_{i A}^{\tilde{\nu} *} \mathcal{U}_{i+6 B}^{\tilde{\nu} *}, \\
\tilde{C}_{A B}^{3} & =-\frac{1}{2} \mathcal{U}_{i+6 A}^{\tilde{\nu}} \mathcal{U}_{i B}^{\tilde{\nu}}, \\
\tilde{C}_{A B}^{6} & =-\frac{1}{2} \mathcal{U}_{i+6 A}^{\tilde{\nu}} \mathcal{U}_{i+6 B}^{\tilde{\nu} *} .
\end{aligned}
$$

In the above, $\mathcal{U}^{\tilde{\nu}}$ is the unitary matrix diagonalizing the sneutrino mass matrix.

Notice that we have factored out the weak coupling constant $g_{w}$ from all interaction vertices defined above. To better identify chirality-flip mass effects in the CLFV amplitudes, we have also pulled out a factor $m_{l} /\left(\sqrt{2} c_{\beta} M_{W}\right)$ from the interaction vertex $\tilde{B}_{\operatorname{lm} A}^{L}$.

\section{Appendix B: LOOP FUNCTIONS}

The CLFV amplitudes are expressed in terms of leading-order one-loop functions. We expand the loop functions with respect to the momenta and masses of the external charged leptons, while keeping only the leading non-zero terms. The leading terms may then be expressed, in terms of the dimensionless loop integrals

$$
\begin{aligned}
\bar{J}_{n_{1} n_{2} \ldots n_{k}}^{m}\left(\lambda_{1}, \lambda_{2}, \ldots, \lambda_{k}\right) & =\frac{\left(\mu^{2}\right)^{2-D / 2}}{\left(M_{W}^{2}\right)^{-D / 2-m+\sum_{i} n_{i}}} \int \frac{d^{D} \ell}{(2 \pi)^{D}} \frac{\left(\ell^{2}\right)^{m}}{\prod_{i=1}^{k}\left(\ell^{2}-m_{i}^{2}\right)^{n_{i}}} \\
& =\frac{i(-1)^{m-\sum_{i} n_{i}}}{(4 \pi)^{D / 2} \Gamma\left(\frac{D}{2}\right)}\left(\frac{\mu^{2}}{M_{W}^{2}}\right)^{2-D / 2} \int_{0}^{\infty} \frac{d x x^{D / 2-1+m}}{\prod_{i=1}^{k}\left(x+\lambda_{i}\right)^{n_{i}}}
\end{aligned}
$$

where $m_{i}$ are loop particle masses, $n_{i}$ are the exponents of the propagator denominators, $\lambda_{i}=m_{i}^{2} / M_{W}^{2}$ are dimensionless mass parameters and $\mu$ is 't Hooft's renormalization mass scale. We choose $\mu$ to be $M_{W}$, even though any other scale can be chosen equally well as a reference scale for any of the integrals. For the amplitudes we have been calculating, the integrals are either divergent and satisfy $m+2-\sum_{i} n_{i}=0$, or they are convergent with $m+2-\sum_{i} n_{i}<0$. For convergent integrals, we may set $D=4$, whilst for divergent integrals we take $D=4-2 \varepsilon$. We pull out a factor $i /(4 \pi)^{2}$ from all integrals. Thus, we have for finite integrals,

$$
\bar{J}_{n_{1} n_{2} \ldots n_{k}}^{m}\left(\lambda_{1}, \lambda_{2}, \ldots, \lambda_{k}\right) \equiv \frac{i}{(4 \pi)^{2}} J_{n_{1} n_{2} \ldots n_{k}}^{m}\left(\lambda_{1}, \lambda_{2}, \ldots, \lambda_{k}\right) .
$$

Instead, the divergent integrals are written down as a sum of a divergent+constant term and a finite mass-dependent term:

$$
\bar{J}_{n_{1} n_{2} \ldots n_{k}}^{m}\left(\lambda_{1}, \lambda_{2}, \ldots \lambda_{k}\right) \equiv \frac{i}{(4 \pi)^{2}}\left(\frac{1}{\varepsilon}+\text { const }+J_{n_{1} n_{2} \ldots n_{k}}^{m}\left(\lambda_{1}, \lambda_{2}, \ldots, \lambda_{k}\right)\right) .
$$

In the CLFV amplitudes, the divergent+constant terms vanish in the total sum, or as a result of a GIM-like mechanism. Therefore, all CLFV amplitudes can be expressed in terms of finite mass dependent functions $J_{n_{1} n_{2} \ldots}^{m}\left(\lambda_{1}, \lambda_{2}, \ldots\right)$, which we call them the basic integrals. The CLFV amplitudes and the corresponding formfactors considered here are described by 9 basic integrals, four for the photonic amplitude: $J_{31}^{0}, J_{31}^{1}, J_{41}^{1}$ and $J_{41}^{2}$, three for the $Z$-boson amplitude: $J_{11}^{0}, J_{111}^{0}$ and $J_{111}^{1}$, and two for box amplitudes: $J_{1111}^{0}$ and $J_{1111}^{1}$. 


\section{Appendix C: ONE-LOOP FORMFACTORS}

Here we present the complete analytical form of the CLFV formfactors $F_{\gamma}, F_{Z}$ and $F_{\text {box }}$ defined in Section III, in the Feynman-'t Hooft gauge. In the following, the usual summation convention over repeated indices is implied. The interaction vertices and loop functions used here are given in Appendices $\mathrm{A}$ and B. respectively.

\section{Photon Formfactors}

The formfactors $F_{\gamma}^{L}, F_{\gamma}^{R}, G_{\gamma}^{L}$ and $G_{\gamma}^{R}$ may be explicitly written as follows:

$$
\begin{aligned}
& \left(F_{\gamma}^{L}\right)_{l^{\prime} l}=F_{l^{\prime} l \gamma}^{N}+F_{l^{\prime} l \gamma}^{L, \tilde{N}}+F_{l^{\prime} l \gamma}^{L, \mathrm{SB}}, \\
& \left(F_{\gamma}^{R}\right)_{l^{\prime} l}=F_{l^{\prime} l \gamma}^{N}+F_{l^{\prime} l \gamma}^{R, \tilde{N}}+F_{l^{\prime} l \gamma}^{R, \mathrm{SB}}, \\
& \left(G_{\gamma}^{L}\right)_{l^{\prime} l}=m_{l^{\prime}}\left(G_{l^{\prime} l \gamma}^{N}+G_{l^{\prime} l \gamma}^{L, N}\right)+G_{l^{\prime} l \gamma}^{L, \mathrm{SB}}, \\
& \left(G_{\gamma}^{R}\right)_{l^{\prime} l}=m_{l}\left(G_{l^{\prime} l \gamma}^{N}+G_{l^{\prime} l \gamma}^{R, \tilde{N}}\right)+G_{l^{\prime} l \gamma}^{R, \mathrm{SB}},
\end{aligned}
$$

where

$$
\begin{aligned}
& F_{l^{\prime} l \gamma}^{N}=B_{l^{\prime} a} B_{l a}^{*}\left[2\left(J_{31}^{1}\left(1, \lambda_{n_{a}}\right)-\frac{1}{6} J_{41}^{2}\left(1, \lambda_{n_{a}}\right)\right)-\frac{1}{6} \lambda_{n_{a}} J_{41}^{2}\left(1, \lambda_{n_{a}}\right)-\frac{1}{6 t_{\beta}^{2}} \lambda_{n_{a}} J_{41}^{2}\left(\lambda_{H^{+}}, \lambda_{n_{a}}\right)\right], \\
& G_{l^{\prime} l \gamma}^{N}=B_{l^{\prime} a} B_{l a}^{*}\left[J_{31}^{1}\left(1, \lambda_{n_{a}}\right)+J_{41}^{2}\left(1, \lambda_{n_{a}}\right)+\lambda_{n_{a}}\left(\frac{1}{2} J_{41}^{1}\left(1, \lambda_{n_{a}}\right)-J_{31}^{0}\left(1, \lambda_{n_{a}}\right)\right)\right. \\
& \left.+\lambda_{n_{a}} \lambda_{H^{+}}\left(\frac{1}{2 t_{\beta}^{2}} J_{41}^{1}\left(\lambda_{H^{+}}, \lambda_{n_{a}}\right)+J_{31}^{0}\left(\lambda_{H^{+}}, \lambda_{n_{a}}\right)\right)\right] \\
& F_{l^{\prime} l \gamma}^{L, \tilde{N}}=\frac{1}{2}\left(\tilde{B}_{l^{\prime} k A}^{R, 1} \tilde{B}_{l k A}^{R *}+\tilde{B}_{l^{\prime} k A}^{R, 2} \tilde{B}_{l k A}^{R, 2 *}\right)\left[-\frac{2}{3} J_{41}^{2}\left(\lambda_{\tilde{\chi}_{k}}, \lambda_{\tilde{N}_{A}}\right)+\lambda_{\tilde{\chi}_{k}} J_{41}^{1}\left(\lambda_{\tilde{\chi}_{k}}, \lambda_{\tilde{N}_{A}}\right)\right], \\
& F_{l^{\prime} l \gamma}^{R, \tilde{N}} \equiv \frac{m_{l} m_{l^{\prime}}}{4 c_{\beta}^{2} M_{W}^{2}}\left(\tilde{B}_{l^{\prime} k A}^{L, 1} \tilde{B}_{l k A}^{L, 1 *}+\tilde{B}_{l^{\prime} k A}^{L, 2} \tilde{B}_{l k A}^{L, 2 *}\right)\left[-\frac{2}{3} J_{41}^{2}\left(\lambda_{\tilde{\chi}_{k}}, \lambda_{\tilde{N}_{A}}\right)+\lambda_{\tilde{\chi}_{k}} J_{41}^{1}\left(\lambda_{\tilde{\chi}_{k}}, \lambda_{\tilde{N}_{A}}\right)\right] \text {, } \\
& G_{l^{\prime} l \gamma}^{L, \tilde{N}}=\frac{1}{2}\left(\tilde{B}_{l^{\prime} k A}^{L, 1} \tilde{B}_{l k A}^{L, 1 *}+\tilde{B}_{l^{\prime} k A}^{L, 2} \tilde{B}_{l k A}^{L, 2 *}\right)\left[-\frac{m_{l}^{2}}{2 c_{\beta}^{2} M_{W}^{2}} \lambda_{\tilde{\chi}_{k}} J_{41}^{1}\left(\lambda_{\tilde{\chi}_{k}}, \lambda_{\tilde{N}_{A}}\right)\right] \\
& +\frac{1}{2}\left(\tilde{B}_{l^{\prime} k A}^{R, 1} \tilde{B}_{l k A}^{R, 1 *}+\tilde{B}_{l^{\prime} k A}^{R, 2} \tilde{B}_{l k A}^{R, 2 *}\right)\left[-\lambda_{\tilde{\chi}_{k}} J_{41}^{1}\left(\lambda_{\tilde{\chi}_{k}}, \lambda_{\tilde{N}_{A}}\right)\right] \\
& +\frac{1}{2}\left(\tilde{B}_{l^{\prime} k A}^{29 L, 1} \tilde{V}_{l k A}^{R, 1 *}+\tilde{B}_{l^{\prime} k A}^{L, 2} \tilde{V}_{l k A}^{R, 2 *}\right)\left[\frac{\sqrt{2}}{c_{\beta}} \sqrt{\lambda_{\tilde{\chi}_{k}}} J_{31}^{1}\left(\lambda_{\tilde{\chi}_{k}}, \lambda_{\tilde{N}_{A}}\right)\right] \text {, } \\
& G_{l^{\prime} l \gamma}^{R, \tilde{N}}=\frac{1}{2}\left(\tilde{B}_{l^{\prime} k A}^{L, 1} \tilde{B}_{l k A}^{L, 1 *}+\tilde{B}_{l^{\prime} k A}^{L, 2} \tilde{B}_{l k A}^{L, 2 *}\right)\left[-\frac{m_{l^{\prime}}^{2}}{2 c_{\beta}^{2} M_{W}^{2}} \lambda_{\tilde{\chi}_{k}} J_{41}^{1}\left(\lambda_{\tilde{\chi}_{k}}, \lambda_{\tilde{N}_{A}}\right)\right] \\
& +\frac{1}{2}\left(\tilde{B}_{l^{\prime} k A}^{R, 1} \tilde{B}_{l k A}^{R, 1 *}+\tilde{B}_{l^{\prime} k A}^{R, 2} \tilde{B}_{l k A}^{R, 2 *}\right)\left[-\lambda_{\tilde{\chi}_{k}} J_{41}^{1}\left(\lambda_{\tilde{\chi}_{k}}, \lambda_{\tilde{N}_{A}}\right)\right] \\
& +\frac{1}{2}\left(\tilde{B}_{l^{\prime} k A}^{R, 1} B_{l k A}^{L, 1 *}+\tilde{B}_{l^{\prime} k A}^{R, 2} \tilde{B}_{l k A}^{L, 2 *}\right)\left[\frac{\sqrt{2}}{c_{\beta}} \sqrt{\lambda_{\tilde{\chi_{k}}}} J_{31}^{1}\left(\lambda_{\tilde{\chi}_{k}}, \lambda_{\tilde{N}_{A}}\right)\right], \\
& F_{l^{\prime} l \gamma}^{L, \mathrm{SB}}=\tilde{V}_{l^{\prime} m a}^{0 \ell R} \tilde{V}_{l m a}^{0 \ell R *}\left[-\frac{1}{3} J_{41}^{2}\left(\lambda_{\tilde{e}_{a}}, \lambda_{\tilde{\chi}_{m}^{0}}\right)\right] \\
& F_{l^{\prime} l \gamma}^{R, \mathrm{SB}}=\tilde{V}_{l^{\prime} m a}^{0 \ell L} \tilde{V}_{l m a}^{0 \ell L *}\left[-\frac{1}{3} J_{41}^{2}\left(\lambda_{\tilde{e}_{a}}, \lambda_{\tilde{\chi}_{m}^{0}}\right)\right] \text {, } \\
& G_{l^{\prime} l \gamma}^{L, \mathrm{SB}}=\tilde{V}_{l^{\prime} m a}^{0 \ell R} \tilde{V}_{l m a}^{0 \ell R *}\left[m_{l^{\prime}} \lambda_{\tilde{e}_{a}} J_{41}^{1}\left(\lambda_{\tilde{e}_{a}}, \lambda_{\tilde{\chi}_{m}^{0}}\right)\right]+\tilde{V}_{l^{\prime} m a}^{0 \ell L} \tilde{V}_{l m a}^{0 \ell L *}\left[m_{l} \lambda_{\tilde{e}_{a}} J_{41}^{1}\left(\lambda_{\tilde{e}_{a}}, \lambda_{\tilde{\chi}_{m}^{0}}\right)\right] \\
& +\tilde{V}_{l^{\prime} m a}^{0 \ell L} \tilde{V}_{l m a}^{0 \ell R *}\left[+2 m_{\tilde{\chi}_{m}^{0}} \lambda_{\tilde{e}_{a}} J_{31}^{0}\left(\lambda_{\tilde{e}_{a}}, \lambda_{\tilde{\chi}_{m}^{0}}\right)\right]
\end{aligned}
$$




$$
\begin{aligned}
G_{l^{\prime} l \gamma}^{R, \mathrm{SB}} & =\tilde{V}_{l^{\prime} m a}^{0 \ell L} \tilde{V}_{l m a}^{0 \ell L *}\left[m_{l^{\prime}} \lambda_{\tilde{e}_{a}} J_{41}^{1}\left(\lambda_{\tilde{e}_{a}}, \lambda_{\tilde{\chi}_{m}^{0}}\right)\right]+\tilde{V}_{l^{\prime} m a}^{0 \ell R} \tilde{V}_{l m a}^{0 \ell R *}\left[m_{l} \lambda_{\tilde{e}_{a}} J_{41}^{1}\left(\lambda_{\tilde{e}_{a}}, \lambda_{\tilde{\chi}_{m}^{0}}\right)\right] \\
& +\tilde{V}_{l^{\prime} m a}^{0 \ell R} \tilde{V}_{l m a}^{0 \ell L *}\left[+2 m_{\tilde{\chi}_{m}^{0}} \lambda_{\tilde{e}_{a}} J_{31}^{0}\left(\lambda_{\tilde{e}_{a}}, \lambda_{\tilde{\chi}_{m}^{0}}\right)\right] .
\end{aligned}
$$

\section{2. $Z$-Boson Formfactors}

The formfactors $F_{Z}^{L}$ and $F_{Z}^{R}$ may be decomposed as follows:

$$
\begin{aligned}
& \left(F_{Z}^{L}\right)_{l^{\prime} l}=F_{l^{\prime} l Z}^{N}+F_{l^{\prime} l Z}^{L, \tilde{N}}+F_{l^{\prime} l Z}^{L, \mathrm{SB}}, \\
& \left(F_{\gamma}^{R}\right)_{l^{\prime} l}=F_{l^{\prime} l Z}^{N}+F_{l^{\prime} l Z}^{R, \tilde{N}}+F_{l^{\prime} l Z}^{R, \mathrm{SB}},
\end{aligned}
$$

where

$$
\begin{aligned}
& F_{l^{\prime} l Z}^{L, N}=B_{l^{\prime} a} B_{l a}^{*}\left[\frac{5}{2} \lambda_{n_{a}} J_{21}^{0}\left(1, \lambda_{n_{a}}\right)\right] \\
& +B_{l^{\prime} b} C_{b a} B_{l a}^{*}\left[-\frac{1}{2} J_{111}^{1}\left(1, \lambda_{n_{b}}, \lambda_{n_{a}}\right)+\frac{1}{2} \lambda_{n_{a}} \lambda_{n_{b}} J_{111}^{0}\left(1, \lambda_{n_{b}}, \lambda_{n_{a}}\right)+\frac{1}{2 t_{\beta}^{2}} \lambda_{n_{a}} \lambda_{n_{b}} J_{111}^{0}\left(\lambda_{H^{+}}, \lambda_{n_{b}}, \lambda_{n_{a}}\right)\right], \\
& F_{l^{\prime} l Z}^{R, N}=-\frac{m_{l} m_{l^{\prime}} t_{\beta}^{2}}{4 M_{W}^{2}} B_{l^{\prime} b} C_{b a} B_{l a}^{*} J_{111}^{1}\left(\lambda_{H^{+}}, \lambda_{n_{b}}, \lambda_{n_{a}}\right), \\
& F_{l^{\prime} l Z}^{L, \tilde{N}}=\frac{1}{2}\left(\tilde{B}_{l^{\prime} m A}^{R, 1} \tilde{V}_{m k}^{\tilde{\chi}^{-} R} \tilde{B}_{l k A}^{R, 1 *}+\tilde{B}_{l^{\prime} m A}^{R, 2} \tilde{V}_{m k}^{\tilde{\chi}^{-} R} \tilde{B}_{l k A}^{R, 2 *}\right) J_{111}^{1}\left(\lambda_{\tilde{\chi}_{m}}, \lambda_{\tilde{\chi}_{k}}, \lambda_{\tilde{N}_{A}}\right) \\
& -\left(\tilde{B}_{l^{\prime} m A}^{R, 1} \tilde{V}_{m k}^{\tilde{\chi}^{-} L} \tilde{B}_{l m A}^{R, 1 *}+\tilde{B}_{l^{\prime} m A}^{R, 2} \tilde{V}_{m k}^{\tilde{\chi}^{-} L} \tilde{B}_{l m A}^{R, 2 *}\right) \sqrt{\lambda_{\tilde{\chi}_{m}} \lambda_{\tilde{\chi}_{k}}} J_{111}^{0}\left(\lambda_{\tilde{\chi}_{m}}, \lambda_{\tilde{\chi}_{k}}, \lambda_{\tilde{N}_{A}}\right) \\
& +\frac{1}{2}\left(\tilde{B}_{l^{\prime} m A}^{R, 1} \tilde{B}_{l k A}^{R, 1 *}+\tilde{B}_{l^{\prime} m A}^{R, 2} \tilde{B}_{l k A}^{R, 2 *}\right)\left(\frac{1}{2}-s_{w}^{2}\right)\left(J_{21}^{1}\left(\lambda_{\tilde{\chi}_{k}}, \lambda_{\tilde{N}_{A}}\right)-2 J_{11}^{0}\left(\lambda_{\tilde{\chi}_{k}}, \lambda_{\tilde{N}_{A}}\right)\right) \\
& +\frac{1}{4}\left(\tilde{B}_{l^{\prime} k A}^{R, 1} \tilde{C}_{B A}^{1} \tilde{B}_{l k A}^{R, 1 *}+\tilde{B}_{l^{\prime} k A}^{R, 1} \tilde{C}_{B A}^{2} \tilde{B}_{l k A}^{R, 2 *}+\tilde{B}_{l^{\prime} m A}^{R, 2} \tilde{C}_{B A}^{3} \tilde{B}_{l k A}^{R, 1 *}\right. \\
& \left.+\tilde{B}_{l^{\prime} m A}^{R, 2} \tilde{C}_{B A}^{4} \tilde{B}_{l k A}^{R, 2 *}\right) J_{111}^{1}\left(\lambda_{\tilde{\chi}_{k}}, \lambda_{\tilde{N}_{B}}, \lambda_{\tilde{N}_{A}}\right) \text {, } \\
& F_{l^{\prime} l Z}^{R, \tilde{N}}=\frac{m_{l} m_{l^{\prime}}}{2 s_{\beta}^{2} M_{W}^{2}}\left[\frac{1}{2}\left(\tilde{B}_{l^{\prime} m A}^{L, 1} \tilde{V}_{m k}^{\tilde{\chi}^{-} L} \tilde{B}_{l k A}^{L, 1 *}+\tilde{B}_{l^{\prime} m A}^{L, 2} \tilde{V}_{m k}^{\tilde{\chi}^{-} L} \tilde{B}_{l k A}^{L, 2 *}\right) J_{111}^{1}\left(\lambda_{\tilde{\chi}_{m}}, \lambda_{\tilde{\chi}_{k}}, \lambda_{\tilde{N}_{A}}\right)\right. \\
& \text { - }\left(\tilde{B}_{l^{\prime} m A}^{L, 1} \tilde{V}_{m k}^{\tilde{\chi}^{-} R} \tilde{B}_{l m A}^{L, 1 *}+\tilde{B}_{l^{\prime} m A}^{L, 2} \tilde{V}_{m k}^{\tilde{\chi}^{-} R} \tilde{B}_{l m A}^{L, 2 *}\right) \sqrt{\lambda_{\tilde{\chi}_{m}} \lambda_{\tilde{\chi}_{k}}} J_{111}^{0}\left(\lambda_{\tilde{\chi}_{m}}, \lambda_{\tilde{\chi}_{k}}, \lambda_{\tilde{N}_{A}}\right) \\
& +\frac{1}{2}\left(\tilde{B}_{l^{\prime} m A}^{L, 1} \tilde{B}_{l k A}^{L, 1 *}+\tilde{B}_{l^{\prime} m A}^{L, 2} \tilde{B}_{l k A}^{L, 2 *}\right)\left(-s_{w}^{2}\right)\left(J_{21}^{1}\left(\lambda_{\tilde{\chi}_{k}}, \lambda_{\tilde{N}_{A}}\right)-2 J_{11}^{0}\left(\lambda_{\tilde{\chi}_{k}}, \lambda_{\tilde{N}_{A}}\right)\right) \\
& +\frac{1}{4}\left(\tilde{B}_{l^{\prime} k A}^{L, 1} \tilde{C}_{B A}^{1} \tilde{B}_{l k A}^{L, 1 *}+\tilde{B}_{l^{\prime} k A}^{L, 1} \tilde{C}_{B A}^{2} \tilde{B}_{l k A}^{L, 2 *}+\tilde{B}_{l^{\prime} m A}^{L, 2} \tilde{C}_{B A}^{3} \tilde{B}_{l k A}^{L, 1 *}\right. \\
& \left.\left.+\tilde{B}_{l^{\prime} m A}^{L, 2} \tilde{C}_{B A}^{4} \tilde{B}_{l k A}^{L, 2 *}\right) J_{111}^{1}\left(\lambda_{\tilde{\chi}_{k}}, \lambda_{\tilde{N}_{B}}, \lambda_{\tilde{N}_{A}}\right)\right], \\
& F_{l^{\prime} l Z}^{L, \mathrm{SB}}=-\tilde{V}_{l^{\prime} m a}^{0 \ell R} \tilde{V}_{m k}^{\tilde{\chi}^{0} R} \tilde{V}_{l k a}^{0 \ell R *} J_{111}^{1}\left(\lambda_{\tilde{\chi}_{m}^{0}}, \lambda_{\tilde{\chi}_{k}^{0}}, \lambda_{\tilde{e}_{a}}\right)+\tilde{V}_{l^{\prime} m a}^{0 \ell R} \tilde{V}_{m k}^{\tilde{\chi}^{0} L} \tilde{V}_{l k a}^{0 \ell R *} 2 \sqrt{\lambda_{\tilde{\chi}_{m}} \lambda_{\tilde{\chi}_{k}}} J_{111}^{0}\left(\lambda_{\tilde{\chi}_{m}^{0}}, \lambda_{\tilde{\chi}_{k}^{0}}, \lambda_{\tilde{e}_{a}}\right) \\
& +\tilde{V}_{l^{\prime} k a}^{0 \ell R} \tilde{V}_{l k a}^{0 \ell R *}\left(\frac{1}{2}-s_{w}^{2}\right)\left(-\frac{1}{2} J_{21}^{1}\left(\lambda_{\tilde{\chi}_{k}^{0}}, \lambda_{\tilde{e}_{a}}\right)+J_{11}^{0}\left(\lambda_{\tilde{\chi}_{k}^{0}}, \lambda_{\tilde{e}_{a}}\right)\right)-\frac{1}{2} \tilde{V}_{l^{\prime} k b}^{0 \ell L} \tilde{V}_{b a}^{\tilde{e}} \tilde{V}_{l k a}^{0 \ell L *} J_{111}^{1}\left(\lambda_{\tilde{\chi}_{k}^{0}}, \lambda_{\tilde{e}_{b}}, \lambda_{\tilde{e}_{a}}\right), \\
& F_{l^{\prime} l Z}^{R, \mathrm{SB}}=-\tilde{V}_{l^{\prime} m a}^{0 \ell L} \tilde{V}_{m k}^{\tilde{\chi}^{0} L} \tilde{V}_{l k a}^{0 \ell L *} J_{111}^{1}\left(\lambda_{\tilde{\chi}_{m}^{0}}, \lambda_{\tilde{\chi}_{k}^{0}}, \lambda_{\tilde{e}_{a}}\right)+\tilde{V}_{l^{\prime} m a}^{0 \ell L} \tilde{V}_{m k}^{\tilde{\chi}^{0} R} \tilde{V}_{l k a}^{0 \ell L *} 2 \sqrt{\lambda_{\tilde{\chi}_{m}} \lambda_{\tilde{\chi}_{k}}} J_{111}^{0}\left(\lambda_{\tilde{\chi}_{m}^{0}}, \lambda_{\tilde{\chi}_{k}^{0}}, \lambda_{\tilde{e}_{a}}\right) \\
& +\tilde{V}_{l^{\prime} k a}^{0 \ell L} \tilde{V}_{l k a}^{0 \ell L *}\left(-s_{w}^{2}\right)\left(-\frac{1}{2} J_{21}^{1}\left(\lambda_{\tilde{\chi}_{k}^{0}}, \lambda_{\tilde{e}_{a}}\right)+J_{11}^{0}\left(\lambda_{\tilde{\chi}_{k}^{0}}, \lambda_{\tilde{e}_{a}}\right)\right)-\frac{1}{2} \tilde{V}_{l^{\prime} k b}^{0 \ell R} \tilde{V}_{b a}^{\tilde{e}} \tilde{V}_{l k a}^{0 \ell R *} J_{111}^{1}\left(\lambda_{\tilde{\chi}_{k}^{0}}, \lambda_{\tilde{e}_{b}}, \lambda_{\tilde{e}_{a}}\right) .
\end{aligned}
$$




\section{Leptonic Box Formfactors}

The leptonic box amplitudes are expressed in terms of the chiral structures: $\bar{l}^{\prime} \Gamma_{A}^{X} l \bar{l}_{1} \Gamma_{A}^{Y} l_{2}^{C}[\mathrm{cf}$. (3.8]. There are two distinct contributions to the chiral amplitudes. The first one has direct relevance to the original structure given above and we denote it with a subscript $D$. The second contribution comes from a chiral amplitude of the form $\bar{l}_{1} \Gamma_{A}^{X} l \bar{l}^{\prime} \Gamma_{A}^{Y} l_{2}^{C}$, which contributes to the original amplitude $\bar{l}^{\prime} \Gamma_{A}^{X} l \bar{l}_{1} \Gamma_{A}^{Y} l_{2}^{C}$, after performing a Fierz transformation. This Fierz-transformed contribution is indicated with a subscript $F$. More explicitly, the leptonic box formfactors are given by

$$
\begin{aligned}
B_{\ell V}^{L L} & =B_{\ell V, D}^{L L}+B_{\ell V, F}^{L L}, & B_{\ell V}^{R R} & =B_{\ell V, D}^{R R}+B_{\ell V, F}^{R R}, \\
B_{\ell V}^{L R} & =B_{\ell V, D}^{L R}-\frac{1}{2} B_{\ell S, F}^{L R}, & B_{\ell V}^{R L} & =B_{\ell V, D}^{R L}-\frac{1}{2} B_{\ell S, F}^{R L}, \\
B_{\ell S}^{L L} & =B_{\ell S, D}^{L L}+\frac{1}{2} B_{\ell S, F}^{L L}+\frac{3}{2} B_{\ell T, F}^{L L}, & B_{\ell S}^{R R} & =B_{\ell S, D}^{R R}+\frac{1}{2} B_{\ell S, F}^{R R}+\frac{3}{2} B_{\ell T, F}^{R R}, \\
B_{\ell S}^{L R} & =B_{\ell S, D}^{L R}-2 B_{\ell V, F}^{L R}, & B_{\ell S}^{R L} & =B_{\ell S, D}^{R L}-2 B_{\ell V, F}^{R L}, \\
B_{\ell T}^{L L} & =B_{\ell T, D}^{L L}-\frac{1}{2} B_{\ell T, F}^{L L}+\frac{1}{2} B_{\ell S, F}^{L L}, & B_{\ell T}^{R R} & =B_{\ell T, D}^{R R}-\frac{1}{2} B_{\ell T, F}^{R R}+\frac{1}{2} B_{\ell S, F}^{R R} .
\end{aligned}
$$

The direct and Fierz-transformed contributions to the formfactors are related by the exchange of outgoing leptons

$$
B_{\ell A, F}^{X Y}=B_{\ell A, D}^{X Y}\left(l^{\prime} \leftrightarrow l_{1}\right)
$$

The direct contributions have direct $N, \mathrm{SB}$ and Fierz-transformed $\tilde{N}$ contributions:

$$
\begin{array}{rlrl}
B_{\ell V, D}^{L L} & =B_{\ell V, D}^{L L, N}+B_{\ell V, F}^{L L, \tilde{N}}+B_{\ell V, D}^{L L, \mathrm{SB}}, & B_{\ell V, D}^{R R} & =B_{\ell V, D}^{R R, \mathrm{SB}}, \\
B_{\ell V, D}^{L R}=B_{\ell V, D}^{L R, \mathrm{SB}}, & B_{\ell V, D}^{R L} & =-\frac{1}{2} B_{\ell S, F}^{R L, \tilde{N}}+B_{\ell V, D}^{R L, \mathrm{SB}}, \\
B_{\ell S, D}^{L L}=B_{\ell S, D}^{L L, \mathrm{SB}}, & B_{\ell S, D}^{R R}=\frac{1}{2} B_{\ell S, F}^{R R, \tilde{N}}+B_{\ell S, D}^{R R, \mathrm{SB}}, \\
B_{\ell S, D}^{L R}=B_{\ell S, D}^{L R, \mathrm{SB}}, & B_{\ell S, D}^{R L}=B_{\ell S, D}^{R L, N}-2 B_{\ell V, F}^{R L, \tilde{N}}+B_{\ell S, D}^{R L, \mathrm{SB}}, \\
B_{\ell T, D}^{L L}=B_{\ell T, D}^{L L, \mathrm{SB}}, & B_{\ell T, D}^{R R}=\frac{1}{2} B_{\ell S, F}^{R R, \tilde{N}}+B_{\ell T, D}^{R R, \mathrm{SB}} .
\end{array}
$$

The formfactor contributions from (C.11) read:

$$
\begin{aligned}
B_{\ell V, D}^{L L, N} & =B_{l a}^{*} B_{l_{2} b}^{*} B_{l^{\prime} a} B_{l_{1} b}\left[-\left(1+\frac{\lambda_{n_{a}} \lambda_{n_{b}}}{4}\right) J_{211}^{1}\left(1, \lambda_{n_{a}}, \lambda_{n_{b}}\right)+2 \lambda_{n_{a}} \lambda_{n_{b}} J_{211}^{0}\left(1, \lambda_{n_{a}}, \lambda_{n_{b}}\right)\right. \\
& -2 \lambda_{n_{a}} \lambda_{n_{b}} t_{\beta}^{-2} J_{1111}^{0}\left(1, \lambda_{H^{+}}, \lambda_{n_{a}}, \lambda_{n_{b}}\right)-\frac{1}{2} \lambda_{a} \lambda_{b} t_{\beta}^{-2} J_{1111}^{1}\left(1, \lambda_{H^{+}}, \lambda_{n_{a}}, \lambda_{n_{b}}\right) \\
& \left.-\frac{1}{4} \lambda_{a} \lambda_{b} t_{\beta}^{-4} J_{211}^{1}\left(\lambda_{H^{+}}, \lambda_{n_{a}}, \lambda_{n_{b}}\right)\right] \\
B_{\ell V, D}^{R L, N}= & -B_{l a}^{*} B_{l_{2} b}^{*} B_{l^{\prime} a} B_{l_{1} b} \frac{m_{l} m_{l_{1}} t_{\beta}^{2}}{M_{W}^{2}}\left(J_{1111}^{1}\left(1, \lambda_{H^{+}}, \lambda_{n_{a}}, \lambda_{n_{b}}\right)+\lambda_{a} \lambda_{b} J_{1111}^{0}\left(\lambda_{H^{+}}, \lambda_{n_{a}}, \lambda_{n_{b}}\right)\right) \\
B_{\ell V, F}^{L L, \tilde{N}}= & \left(\tilde{B}_{l_{1} k B}^{R, 1} \tilde{B}_{l_{2} m B}^{R, 1 *}+\tilde{B}_{l_{1} k B}^{R, 2} \tilde{B}_{l_{2} m B}^{R, 2 *}\right)\left(\tilde{B}_{l^{\prime} m A}^{R, 1} \tilde{B}_{l k A}^{R, 1 *}+\tilde{B}_{l^{\prime} m A}^{R, 2} \tilde{B}_{l k A}^{R, 2 *}\right) J_{1111}^{1}\left(\lambda_{\tilde{\chi}_{k}}, \lambda_{\tilde{\chi}_{m}}, \lambda_{\tilde{N}_{A}}, \lambda_{\tilde{N}_{B}}\right) \\
B_{\ell V, F}^{R L, N}= & \left(\tilde{B}_{l_{1} k B}^{L, 1} \tilde{B}_{l_{2} m B}^{R, 1 *}+\tilde{B}_{l_{1} k B}^{L, 2} \tilde{B}_{l_{2} m B}^{R, 2 *}\right)\left(\tilde{B}_{l^{\prime} m A}^{R, 1} \tilde{B}_{l k A}^{L, 1 *}+\tilde{B}_{l^{\prime} m A}^{R, 2} \tilde{B}_{l k A}^{L, 2 *}\right) \\
\times & \frac{m_{l^{\prime}} m_{l}}{2 c_{\beta}^{2} M_{W}^{2}} J_{1111}^{1}\left(\lambda_{\tilde{\chi}_{k}}, \lambda_{\tilde{\chi}_{m}}, \lambda_{\tilde{N}_{A}}, \lambda_{\tilde{N}_{B}}\right), \\
B_{\ell S, F}^{R R, \tilde{N}}= & \left(\tilde{B}_{l_{1} k B}^{R, 1} \tilde{B}_{l_{2} m B}^{L, 1 *}+\tilde{B}_{l_{1} k B}^{R, 2} \tilde{B}_{l_{2} m B}^{L, 2 *}\right)\left(\tilde{B}_{l^{\prime} m A}^{R, 1} \tilde{B}_{l k A}^{L, 1 *}+\tilde{B}_{l^{\prime} m A}^{R, 2} \tilde{B}_{l k A}^{L, 2 *}\right) \\
\times & \frac{2 m_{l_{2}} m_{l}}{c_{\beta}^{2} M_{W}^{2}} \sqrt{\lambda_{\tilde{\chi}_{k}} \lambda_{\tilde{\chi}_{m}}} J_{1111}^{0}\left(\lambda_{\tilde{\chi}_{k}}, \lambda_{\tilde{\chi}_{m}}, \lambda_{\tilde{N}_{A}}, \lambda_{\tilde{N}_{B}}\right) \\
B_{\ell S, F}^{R L, \tilde{N}}= & \left(\tilde{B}_{l_{1} k B}^{R, 1} \tilde{B}_{l_{2} m B}^{R, 1 *}+\tilde{B}_{l_{1} k B}^{R, 2} \tilde{B}_{l_{2} m B}^{R, 2 *}\right)\left(\tilde{B}_{l^{\prime} m A}^{L, 1} \tilde{B}_{l k A}^{L, 1 *}+\tilde{B}_{l^{\prime} m A}^{L, 2} \tilde{B}_{l k A}^{L, 2 *}\right)
\end{aligned}
$$




$$
\begin{aligned}
& \times \frac{2 m_{l_{1}} m_{l}}{c_{\beta}^{2} M_{W}^{2}} \sqrt{\lambda_{\tilde{\chi}_{k}} \lambda_{\tilde{\chi}_{m}}} J_{1111}^{0}\left(\lambda_{\tilde{\chi}_{k}}, \lambda_{\tilde{\chi}_{m}}, \lambda_{\tilde{N}_{A}}, \lambda_{\tilde{N}_{B}}\right) \\
& B_{\ell V, D}^{L L, \mathrm{SB}}=-\tilde{V}_{l_{1} m b}^{0 \ell R} \tilde{V}_{l m a}^{0 \ell R *} \tilde{V}_{l^{\prime} n a}^{0 \ell R} \tilde{V}_{l_{2} n b}^{0 \ell R *} J_{1111}^{1}\left(\lambda_{\tilde{\chi}_{m}^{0}}, \lambda_{\tilde{\chi}_{n}^{0}}, \lambda_{\tilde{e}_{a}}, \lambda_{\tilde{e}_{b}}\right) \\
& -2 \tilde{V}_{l_{2} m b}^{0 \ell R} \tilde{V}_{l m a}^{0 \ell R *} \tilde{V}_{l^{\prime} n a}^{0 \ell R} \tilde{V}_{l_{1} n b}^{0 \ell R *} \sqrt{\lambda_{\tilde{\chi}_{m}^{0}} \lambda_{\tilde{\chi}_{k}^{0}}} J_{1111}^{0}\left(\lambda_{\tilde{\chi}_{m}^{0}}, \lambda_{\tilde{\chi}_{n}^{0}}, \lambda_{\tilde{e}_{a}}, \lambda_{\tilde{e}_{b}}\right), \\
& B_{\ell V, D}^{R R, \mathrm{SB}}=-\tilde{V}_{l_{1} m b}^{0 \ell L} \tilde{V}_{l m a}^{0 \ell L *} \tilde{V}_{l^{\prime} n a}^{0 \ell L} \tilde{V}_{l_{2} n b}^{0 \ell L *} J_{1111}^{1}\left(\lambda_{\tilde{\chi}_{m}^{0}}, \lambda_{\tilde{\chi}_{n}^{0}}, \lambda_{\tilde{e}_{a}}, \lambda_{\tilde{e}_{b}}\right) \\
& -2 \tilde{V}_{l_{2} m b}^{0 \ell L} \tilde{V}_{l m a}^{0 \ell L *} \tilde{V}_{l^{\prime} n a}^{0 \ell L} \tilde{V}_{l_{1} n b}^{0 \ell L *} \sqrt{\lambda_{\tilde{\chi}_{m}^{0}} \lambda_{\tilde{\chi}_{k}^{0}}} J_{1111}^{0}\left(\lambda_{\tilde{\chi}_{m}^{0}}, \lambda_{\tilde{\chi}_{n}^{0}}, \lambda_{\tilde{e}_{a}}, \lambda_{\tilde{e}_{b}}\right), \\
& B_{\ell V, D}^{L R, \mathrm{SB}}=2 \tilde{V}_{l_{1} m b}^{0 \ell L} \tilde{V}_{l m a}^{0 \ell R *} \tilde{V}_{l^{\prime} n a}^{0 \ell R} \tilde{V}_{l_{2} n b}^{0 \ell L *} \sqrt{\lambda_{\tilde{\chi}_{m}^{0}} \lambda_{\tilde{\chi}_{k}^{0}}} J_{1111}^{0}\left(\lambda_{\tilde{\chi}_{m}^{0}}, \lambda_{\tilde{\chi}_{n}^{0}}, \lambda_{\tilde{e}_{a}}, \lambda_{\tilde{e}_{b}}\right) \\
& +\tilde{V}_{l_{2} m b}^{0 \ell L} \tilde{V}_{l m a}^{0 \ell R *} \tilde{V}_{l^{\prime} n a}^{0 \ell R} \tilde{V}_{l_{1} n b}^{0 \ell L *} J_{1111}^{1}\left(\lambda_{\tilde{\chi}_{m}^{0}}, \lambda_{\tilde{\chi}_{n}^{0}}, \lambda_{\tilde{e}_{a}}, \lambda_{\tilde{e}_{b}}\right), \\
& B_{\ell V, D}^{R L, \mathrm{SB}}=2 \tilde{V}_{l_{1} m b}^{0 \ell R} \tilde{V}_{l m a}^{0 \ell L *} \tilde{V}_{l^{\prime} n a}^{0 \ell L} \tilde{V}_{l_{2} n b}^{0 \ell R *} \sqrt{\lambda_{\tilde{\chi}_{m}^{0}} \lambda_{\tilde{\chi}_{k}^{0}}} J_{1111}^{0}\left(\lambda_{\tilde{\chi}_{m}^{0}}, \lambda_{\tilde{\chi}_{n}^{0}}, \lambda_{\tilde{e}_{a}}, \lambda_{\tilde{e}_{b}}\right) \\
& +\tilde{V}_{l_{2} m b}^{0 \ell R} \tilde{V}_{l m a}^{0 \ell L *} \tilde{V}_{l^{\prime} n a}^{0 \ell} \tilde{V}_{l_{1} n b}^{0 \ell R *} J_{1111}^{1}\left(\lambda_{\tilde{\chi}_{m}^{0}}, \lambda_{\tilde{\chi}_{n}^{0}}, \lambda_{\tilde{e}_{a}}, \lambda_{\tilde{e}_{b}}\right), \\
& B_{\ell S, D}^{L L, \mathrm{SB}}=-2 \tilde{V}_{l_{1} m b}^{0 \ell L} \tilde{V}_{l m a}^{0 \ell R *} \tilde{V}_{l^{\prime} n a}^{0 \ell} \tilde{V}_{l_{2} n b}^{0 \ell R *} \sqrt{\lambda_{\tilde{\chi}_{m}^{0}} \lambda_{\tilde{\chi}_{k}^{0}}} J_{1111}^{0}\left(\lambda_{\tilde{\chi}_{m}^{0}}, \lambda_{\tilde{\chi}_{n}^{0}}, \lambda_{\tilde{e}_{a}}, \lambda_{\tilde{e}_{b}}\right) \\
& -2 \tilde{V}_{l_{2} m b}^{0 \ell R} \tilde{V}_{l m a}^{0 \ell R *} \tilde{V}_{l^{\prime} n a}^{0 \ell L} \tilde{V}_{l_{1} n b}^{0 \ell L *} \sqrt{\lambda_{\tilde{\chi}_{m}^{0}} \lambda_{\tilde{\chi}_{k}^{0}}} J_{1111}^{0}\left(\lambda_{\tilde{\chi}_{m}^{0}}, \lambda_{\tilde{\chi}_{n}^{0}}, \lambda_{\tilde{e}_{a}}, \lambda_{\tilde{e}_{b}}\right), \\
& B_{\ell S, D}^{R R, \mathrm{SB}}=-2 \tilde{V}_{l_{1} m b}^{0 \ell R} \tilde{V}_{l m a}^{0 \ell L *} \tilde{V}_{l^{\prime} n a}^{0 \ell R} \tilde{V}_{l_{2} n b}^{0 \ell L *} \sqrt{\lambda_{\tilde{\chi}_{m}^{0}} \lambda_{\tilde{\chi}_{k}^{0}}} J_{1111}^{0}\left(\lambda_{\tilde{\chi}_{m}^{0}}, \lambda_{\tilde{\chi}_{n}^{0}}, \lambda_{\tilde{e}_{a}}, \lambda_{\tilde{e}_{b}}\right) \\
& -2 \tilde{V}_{l_{2} m b}^{0 \ell L} \tilde{V}_{l m a}^{0 \ell L *} \tilde{V}_{l^{\prime} n a}^{0 \ell R} \tilde{V}_{l_{1} n b}^{0 \ell R *} \sqrt{\lambda_{\tilde{\chi}_{m}^{0}} \lambda_{\tilde{\chi}_{k}^{0}}} J_{1111}^{0}\left(\lambda_{\tilde{\chi}_{m}^{0}}, \lambda_{\tilde{\chi}_{n}^{0}}, \lambda_{\tilde{e}_{a}}, \lambda_{\tilde{e}_{b}}\right), \\
& B_{\ell S, D}^{L R, \mathrm{SB}}=2 \tilde{V}_{l_{1} m b}^{0 \ell R} \tilde{V}_{l m a}^{0 \ell R *} \tilde{V}_{l^{\prime} n a}^{0 \ell L} \tilde{V}_{l_{2} n b}^{0 \ell L *} J_{1111}^{1}\left(\lambda_{\tilde{\chi}_{m}^{0}}, \lambda_{\tilde{\chi}_{n}^{0}}, \lambda_{\tilde{e}_{a}}, \lambda_{\tilde{e}_{b}}\right) \\
& +2 \tilde{V}_{l_{2} m b}^{0 \ell L} \tilde{V}_{l m a}^{0 \ell R *} \tilde{V}_{l^{\prime} n a}^{0 \ell L} \tilde{V}_{l_{1} n b}^{0 \ell R *} J_{1111}^{1}\left(\lambda_{\tilde{\chi}_{m}^{0}}, \lambda_{\tilde{\chi}_{n}^{0}}, \lambda_{\tilde{e}_{a}}, \lambda_{\tilde{e}_{b}}\right), \\
& B_{\ell S, D}^{R L, \mathrm{SB}}=2 \tilde{V}_{l_{1} m b}^{0 \ell L} \tilde{V}_{l m a}^{0 \ell L *} \tilde{V}_{l^{\prime} n a}^{0 \ell R} \tilde{V}_{l_{2} n b}^{0 \ell R *} J_{1111}^{1}\left(\lambda_{\tilde{\chi}_{m}^{0}}, \lambda_{\tilde{\chi}_{n}^{0}}, \lambda_{\tilde{e}_{a}}, \lambda_{\tilde{e}_{b}}\right) \\
& +2 \tilde{V}_{l_{2} m b}^{0 \ell R} \tilde{V}_{l m a}^{0 \ell L *} \tilde{V}_{l^{\prime} n a}^{0 \ell R} \tilde{V}_{l_{1} n b}^{0 \ell L *} J_{1111}^{1}\left(\lambda_{\tilde{\chi}_{m}^{0}}, \lambda_{\tilde{\chi}_{n}^{0}}, \lambda_{\tilde{e}_{a}}, \lambda_{\tilde{e}_{b}}\right), \\
& B_{\ell T, D}^{L L, S B}=-2 \tilde{V}_{l_{1} m b}^{0 \ell L} \tilde{V}_{l m a}^{0 \ell R *} \tilde{V}_{l^{\prime} n a}^{0 \ell} \tilde{V}_{l_{2} n b}^{0 \ell R *} \sqrt{\lambda_{\tilde{\chi}_{m}^{0}} \lambda_{\tilde{\chi}_{k}^{0}}} J_{1111}^{0}\left(\lambda_{\tilde{\chi}_{m}^{0}}, \lambda_{\tilde{\chi}_{n}^{0}}, \lambda_{\tilde{e}_{a}}, \lambda_{\tilde{e}_{b}}\right) \\
& +2 \tilde{V}_{l_{2} m b}^{0 \ell R} \tilde{V}_{l m a}^{0 \ell R *} \tilde{V}_{l^{\prime} n a}^{0 \ell L} \tilde{V}_{l_{1} n b}^{0 \ell L *} \sqrt{\lambda_{\tilde{\chi}_{m}^{0}} \lambda_{\tilde{\chi}_{k}^{0}}} J_{1111}^{0}\left(\lambda_{\tilde{\chi}_{m}^{0}}, \lambda_{\tilde{\chi}_{n}^{0}}, \lambda_{\tilde{e}_{a}}, \lambda_{\tilde{e}_{b}}\right), \\
& B_{\ell T, D}^{R R, \mathrm{SB}}=-2 \tilde{V}_{l_{1} m b}^{0 \ell R} \tilde{V}_{l m a}^{0 \ell L *} \tilde{V}_{l^{\prime} n a}^{0 \ell R} \tilde{V}_{l_{2} n b}^{0 \ell L *} \sqrt{\lambda_{\tilde{\chi}_{m}^{0}} \lambda_{\tilde{\chi}_{k}^{0}}} J_{1111}^{0}\left(\lambda_{\tilde{\chi}_{m}^{0}}, \lambda_{\tilde{\chi}_{n}^{0}}, \lambda_{\tilde{e}_{a}}, \lambda_{\tilde{e}_{b}}\right) \\
& +2 \tilde{V}_{l_{2} m b}^{0 \ell L} \tilde{V}_{l m a}^{0 \ell L *} \tilde{V}_{l^{\prime} n a}^{0 \ell R} \tilde{V}_{l_{1} n b}^{0 \ell R *} \sqrt{\lambda_{\tilde{\chi}_{m}^{0}} \lambda_{\tilde{\chi}_{k}^{0}}} J_{1111}^{0}\left(\lambda_{\tilde{\chi}_{m}^{0}}, \lambda_{\tilde{\chi}_{n}^{0}}, \lambda_{\tilde{e}_{a}}, \lambda_{\tilde{e}_{b}}\right) .
\end{aligned}
$$

\section{Semileptonic Box Formfactors}

Semileptonic form factors have only direct contributions, with the following $N, \tilde{N}$ and SB content:

$$
\begin{aligned}
& B_{d V}^{L L}=B_{d V}^{L L, N}+B_{d V}^{L L, \tilde{N}}+B_{d V}^{L L, \mathrm{SB}} \\
& B_{u V}^{L L}=B_{u V}^{L L, N}+B_{u V}^{L L, \tilde{N}}+B_{u V}^{L L, \mathrm{SB}}
\end{aligned}
$$

and

$$
B_{d A}^{X Y}=B_{d A}^{X Y, \mathrm{SB}}, \quad B_{u A}^{X Y}=B_{u A}^{X Y, \mathrm{SB}},
$$

for $(X, Y, A) \neq(L, L, V)$. The $N$ and $\tilde{N}$ contributions are given by

$$
\begin{aligned}
B_{d V}^{L L, N} & =B_{l^{\prime} a} B_{l a}^{*}\left(V^{*}\right)_{b d_{1}}(V)_{b d_{2}}\left[-\left(1+\frac{\lambda_{n_{a}} \lambda_{u_{b}}}{4}\right) J_{211}^{1}\left(1, \lambda_{n_{a}}, \lambda_{u_{b}}\right)+2 \lambda_{n_{a}} \lambda_{u_{b}} J_{211}^{0}\left(1, \lambda_{n_{a}}, \lambda_{u_{b}}\right)\right. \\
& +\frac{1}{2 t_{\beta}^{2}} \lambda_{n_{a}} \lambda_{u_{b}} J_{1111}^{0}\left(1, \lambda_{H^{+}}, \lambda_{n_{a}}, \lambda_{u_{b}}\right)-\frac{1}{2 t_{\beta}^{2}} \lambda_{n_{a}} \lambda_{u_{b}} J_{1111}^{1}\left(1, \lambda_{H^{+}}, \lambda_{n_{a}}, \lambda_{u_{b}}\right)
\end{aligned}
$$




$$
\begin{aligned}
& \left.-\frac{1}{4 t_{\beta}^{4}} \lambda_{n_{a}} \lambda_{u_{b}} J_{211}^{1}\left(\lambda_{H^{+}}, \lambda_{n_{a}}, \lambda_{u_{b}}\right)\right] \\
B_{u V}^{L L, N} & =B_{l^{\prime} a} B_{l a}^{*}\left(V^{*}\right)_{d_{2} b}(V)_{d_{1} b}\left[\left(4+\frac{\lambda_{n_{a}} \lambda_{d_{b}}}{4}\right) J_{211}^{1}\left(1, \lambda_{n_{a}}, \lambda_{d_{b}}\right)-2 \lambda_{n_{a}} \lambda_{d_{b}} J_{211}^{0}\left(1, \lambda_{n_{a}}, \lambda_{d_{b}}\right)\right. \\
& +\frac{1}{2} \lambda_{n_{a}} \lambda_{d_{b}} J_{1111}^{0}\left(1, \lambda_{H^{+}}, \lambda_{n_{a}}, \lambda_{d_{b}}\right)-\frac{1}{2} \lambda_{n_{a}} \lambda_{d_{b}} J_{1111}^{1}\left(1, \lambda_{H^{+}}, \lambda_{n_{a}}, \lambda_{d_{b}}\right) \\
& \left.+\frac{1}{4} \lambda_{n_{a} \lambda_{d_{b}}} J_{211}^{1}\left(\lambda_{H^{+}}, \lambda_{n_{a}}, \lambda_{d_{b}}\right)\right] \\
B_{d V}^{L L, \tilde{N}} & =-\frac{1}{2} \tilde{V}_{d_{1} k a}^{-d R} \tilde{V}_{d_{2} m a}^{-d R *}\left(\tilde{V}_{l k A}^{-\ell R, 1 *} \tilde{V}_{l^{\prime} m A}^{-\ell R, 1}+\tilde{V}_{l k A}^{-\ell R, 2 *} \tilde{V}_{l^{\prime} m A}^{-\ell R, 2}\right) J_{1111}^{1}\left(\lambda_{\lambda_{\tilde{\chi}_{k}}}, \lambda_{\tilde{\chi}_{m}}, \lambda_{\tilde{N}_{A}}, \lambda_{\tilde{u}_{a}}\right), \\
B_{u V}^{L L, \tilde{N}} & =-\tilde{V}_{u_{1} k a}^{-u R} \tilde{V}_{u_{2} m a}^{-u R *}\left(\tilde{V}_{l k A}^{-\ell R, 1 *} \tilde{V}_{l^{\prime} m A}^{-\ell R, 1}+\tilde{V}_{l k A}^{-\ell R, 2 *} \tilde{V}_{l^{\prime} m A}^{-\ell R, 2}\right) \sqrt{\lambda_{\tilde{\chi}_{k}} \lambda_{\tilde{\chi}_{m}}} J_{1111}^{0}\left(\lambda_{\lambda_{\tilde{\chi}_{k}}}, \lambda_{\tilde{\chi}_{m}}, \lambda_{\tilde{N}_{A}}, \lambda_{\tilde{d}_{a}}\right) .
\end{aligned}
$$

The SB form factors $B_{d A}^{X Y, \mathrm{SB}}$ and $B_{u A}^{X Y, \mathrm{SB}}$, with $X=L, R, Y=L, R$ and $A=V, S, T$, are obtained from the direct leptonic formfactors $B_{\ell A}^{X Y, \mathrm{SB}}$, by making the replacements: $\ell \rightarrow d, l_{1} \rightarrow d, l_{2} \rightarrow d, \tilde{e} \rightarrow \tilde{d}$ and $\ell \rightarrow u, l_{1} \rightarrow u, l_{2} \rightarrow u$, $\tilde{e} \rightarrow \tilde{u}$, in both the interaction vertices and the arguments of the $J$-loop functions that carry the index $b$ in (C.14).

[1] B. T. Cleveland et al. [Homeostake], Astrophys. J. 498 (1998) 505;

J. N. Abdurashitov et al. [SAGE Collaboration], Phys. Rev. C 80 (2009) 015807 [arXiv:0901.2200 [nucl-ex]];

J. Hosaka et al. [Super-Kamiokande Collaboration] Phys. Rev. D 73 (2006) 112001 hep-ex/0508053;

J. P. Cravens et al. [Super-Kamiokande Collaboration], Phys. Rev. D 78 (2008) 032002 [arXiv:0801.0776 [hep-ex]];

B. Aharmim et al. [SNO Collaboration], Phys. Rev. C 72 (2005) 055502;

B. Aharmim et al. [SNO Collaboration], Phys. Rev. C 81 (2010) 055504.

[2] Y. Fukuda et al. [Super-Kamiokande collaboration], Phys. Rev. Lett. 81 (1998) 1562;

J. Hosaka et al. [Super-Kamiokande Collaboration], Phys. Rev. D 74 (2006) 032002 hep-ex/0604011];

J. Ashie et al. [Super-Kamiokande Collaboration], Phys. Rev. D 71 (2005) 112005 hep-ex/0501064].

[3] M.H. Ahn et al. [K2K Collaboration], Phys. Rev. D 74 (2006) 072003 hep-ex/0606032;

P. Adamson et al. [MINOS Collaboration], Phys. Rev. Lett. 101 (2008) 131802 [arXiv:0806.2237] [hep-ex]];

P. Adamson et al. [MINOS Collaboration], Phys. Rev. Lett. 101 (2008) 221804 arXiv:0807.2424 [hep-ex]];

A. Habig et al. [MINOS Collaboration], Nucl. Phys. Proc. Suppl. 218 (2011) 320;

M. Hartz [T2K Collaboration], arXiv:1201.1846 [hep-ex];

P. Adamson et al. [MINOS Collaboration], Phys. Rev. Lett. 108 (2012) 191801, arXiv:1202.2772 [hep-ex]].

[4] S. Abe et al. [KamLAND Collaboration], Phys. Rev. Lett. 100 (2008) 221803.

[5] Y. Abe et al. [DOUBLE-CHOOZ Collaboration], Phys. Rev. Lett. 108 (2012) 131801 arXiv:1112.6353 [hep-ex]];

F. P. An et al. [DAYA-BAY Collaboration], Phys. Rev. Lett. 108 (2012) 171803 arXiv:1203.1669 [hep-ex]];

J. K. Ahn et al. [RENO Collaboration], Phys. Rev. Lett. 108 (2012) 191802 arXiv:1204.0626 [hep-ex]].

[6] B. Pontecorvo, Zh. Eksp. Teor. Fiz. 33 (1957) 549 [JETP 6 (1957) 429];

Z. Maki, M. Nakagawa and S. Sakata, Prog. Theor. Phys. 28 (1962) 870.

[7] J. Adam et al. [MEG Collaboration], Phys. Rev. Lett. 107 (2011) 171801 arXiv:1107.5547 [hep-ex]].

[8] U. Bellgart et al. [SINDRUM Collaboration], Nucl. Phys. B 29919881.

[9] C. Dohmen et al. [SINDRUM II collaboration], Phys. Lett. B 317 (1993) 631.

[10] W. Bertl et al., Eur. Phys. J. C 47 (2006) 337.

[11] Y. Miyazaki et al. [Belle Collaboration], arXiv:1206.5595] [hep-ex];

Y. Miyazaki et al. [Belle Collaboration], Phys. Lett. B 699 (2011) 251 arXiv:1101.0755 [hep-ex]];

B. Aubert et al. [BaBar Collaboration], Phys. Rev. Lett. 103 (2009) 021801;

K. Hayasaka et al. [Belle Collaboration], Phys. Lett. B 687 (2010) 139 [arXiv:1001.3221] [hep-ex]];

J. P. Lees et al. [BaBar Collaboration], Phys. Rev. D 81 (2010) 111101 arXiv:1002.4550 [hep-ex]];

Y. Miyazaki et al. [Belle Collaboration], Phys. Lett. B 692 (2010) 4 arXiv:1003.1183 [hep-ex]];

B. Aubert et al. [BaBar Collaboration], Phys. Rev. Lett. 104 (2010) 151802 arXiv:0908.2381 [hep-ex]];

J. Beringer et al. [Particle Data Group], Phys. Rev. D 86 (2012) 010001.

[12] B. A. Golden, PhD Thesis, 2012, http://meg.web.psi.ch/docs/theses/BenThesis.pdf;

J. Adam, PhD Thesis, 2012, http://meg.web.psi.ch/docs/theses/PhDThesisAdam2012.pdf

[13] J. L. Hewett et al., 1205.2671 [hep-ex].

[14] N. Berger [ $\mu 3$ e Collaboration], arXiv:1110.1504 [hep-ex].

[15] A. Kurup [COMET Collaboration], Nucl. Phys. Proc. Suppl. 218, 38 (2011);

R. Akhmetshin et al. [COMET] http://j-parc.jp/researcher/Hadron/en/pac1203/pdf/COMET-PhaseI-LoI.pdf.

[16] R. J. Abrams et al. [Mu2e Collaboration], arXiv:1211.7019 [physics.ins-det]; 
R. K. Kurschke, arXiv:1112.0242 [hep-ex];

E. C. Dukes, Nucl. Phys. Proc. Suppl. 218, 32 (2011).

[17] Y. Kuno, Nucl. Phys. Proc. Suppl. 149 (2005) 376;

R. J. Barow, Nucl. Phys. Proc. Suppl. 218 (2011) 44.

[18] K. Hayasaka, Journal of Physics: Conference Series 171 (2009) 012079.

[19] M. Bona et al. [SuperB Collaboration] Pisa, Italy: INFN (2007) 453 p. www.pi.infn.it/SuperB/?q=CDR arXiv:0709.0451 [hep-ex]]. With great dismay, we have recently been informed that the SuperB project will be discontinued.

[20] For reviews, see, H.P. Nilles, Phys. Rept. 110 (1984) 1;

H. Haber and G. Kane, Phys. Rept. 117 (1985) 75.

[21] G. Aad et al., 1207.7214 [hep-ex]

[22] S. Chatrchyan et al., 1207.7235 [hep-ex]

[23] P. Minkowski, Phys. Lett. B 67 (1977) 421;

M. Gell-Mann, P. Ramond and R. Slansky, in Supergravity, eds. D.Z. Freedman and P. van Nieuwenhuizen (North-Holland, Amsterdam, 1979);

T. Yanagida, in Proc. of the Workshop on the Unified Theory and the Baryon Number in the Universe, Tsukuba, Japan, 1979, eds. O. Sawada and A. Sugamoto;

S.L. Glashow, in Quarks and Leptons, Cargèse 1979, eds. M. Lévy, et al., (Plenum 1980 New York), p. 707;

R. N. Mohapatra and G. Senjanović, Phys. Rev. Lett. 44 (1980) 912;

J. Schechter and J. W. F. Valle, Phys. Rev. D 22 (1980) 2227.

[24] W. Konetschny and W. Kummer, Phys. Lett. B 70 (1977) 433;

J. Schechter and J. W. F. Valle, Phys. Rev. D 22 (1980) 2227;

G. Lazarides, Q. Shafi and C. Wetterrich, Nucl. Phys. B 181 (1981) 287;

R. N. Mohapatra and G. Senjanovic, Phys. Rev. D 23 (1981) 165;

T. P. Cheng and L.-F. Li, Phys. Rev. D 22 (1980) 2860;

J. Schechter, J. W. F. Valle, Phys. Rev. D 25 (1982) 774.

[25] R. Foot, H. Lew, F.G. He and G.C. Joshi, Z. Phys. C 44 (1989) 441.

[26] D. Wyler and L. Wolfenstein, Nucl. Phys. B 218 (1983) 205.

[27] R. N. Mohapatra, Phys. Rev. Lett. 56 (1986) 561;

R. N. Mohapatra and J. W. F. Valle, Phys. Rev. D 34 (1986) 1642;

S. Nandi and U. Sarkar, Phys. Rev. Lett. 56 (1986) 564.

[28] G. C. Branco, W. Grimus and L. Lavoura, Nucl. Phys. B 312 (1989) 492.

[29] A. Pilaftsis, Z. Phys. C 55 (1992) 275;

P. S. B. Dev and A. Pilaftsis, Phys. Rev. D 86 (2012) 113001 arXiv:1209.4051 [hep-ph]].

[30] A. Pilaftsis, Phys. Rev. Lett. 95 (2005) 081602 hep-ph/0408103;

A. Pilaftsis and T. E. J. Underwood, Phys. Rev. D 72 (2005) 113001 hep-ph/0506107];

F. F. Deppisch and A. Pilaftsis, Phys. Rev. D 83 (2011) 076007 arXiv:1012.1834 [hep-ph]].

[31] E. Nardi, E. Roulet and D. Tommasini Phys. Lett. B 327 (1994) 319 hep-ph/9402224;

S. Antusch, J. P. Baumann and E. Fernández-Martinez, Nucl. Phys. B 810 (2008) 369 [arXiv:0807.1003 [hep-ph]].

[32] S. Bergmann and A. Kagan, Nucl. Phys. B 538 (1999) 368;

F. del Aguila, J. de Blas and M. Perez-Victoria, Phys. Rev. D 78 (2008) 013010.

[33] A. Ilakovac and A. Pilaftsis, Nucl. Phys. B 437 (1995) 491 hep-ph/9403398.

[34] A. Ilakovac and A. Pilaftsis, Phys. Rev. D 80 (2009) 091902 arXiv:0904.2381 [hep-ph]].

[35] A. Ilakovac and A. Pilaftsis, Nucl. Phys. Proc. Suppl. 218 (2011) 26 arXiv:1012.2823] [hep-ph]].

[36] F. Borzumati and A. Masiero, Phys. Rev. Lett. 57 (1986) 961.

[37] J. Hisano, T. Moroi, K. Tobe and M. Yamaguchi, Phys. Rev. D 53 (1996) 2442 hep-ph/9510309.

[38] J. Hisano, T. Moroi, K. Tobe, M. Yamaguchi and T. Yanagida, Phys. Lett. B 357 (1995) 579 hep-ph/9501407;

J. Hisano and D. Nomura, Phys. Rev. D 59 (1999) 116005 hep-ph/9810479;

D. F. Carvalho, J. R. Ellis, M. E. Gomez and S. Lola, Phys. Lett. B 515 (2001) 323 hep-ph/0103256;

J. Hisano, M. Nagai, P. Paradisi and Y. Shimizu, JHEP 0912 (2009) 030 arXiv:0904.2080 [hep-ph]].

[39] J. R. Ellis, J. Hisano, S. Lola and M. Raidal, Nucl. Phys. B 621 (2002) 208 hep-ph/0109125.

[40] C. Arina, F. Bazzocchi, N. Fornengo, J. C. Romao and J. W. F. Valle, Phys. Rev. Lett. 101 (2008) 161802 arXiv:0806.3225 [hep-ph]];

F. Deppisch and A. Pilaftsis, JHEP 0810 (2008) 080 arXiv:0808.0490 [hep-ph]];

F. -X. Josse-Michaux and E. Molinaro, Phys. Rev. D 84 (2011) 125021 arXiv:1108.0482 [hep-ph]];

H. An, P. S. B. Dev, Y. Cai and R. N. Mohapatra, Phys. Rev. Lett. 108 (2012) 081806 [arXiv:1110.1366 [hep-ph]];

B. Dumont, G. Belanger, S. Fichet, S. Kraml and T. Schwetz, JCAP 1209 (2012) 013 [arXiv:1206.1521 [hep-ph]].

[41] A. Pilaftsis, Phys. Rev. D 56 (1997) 5431 hep-ph/9707235;

A. Pilaftsis and T. E. J. Underwood, Nucl. Phys. B 694 (2004) 303 hep-ph/0309342.

[42] T. Cohen, D. E. Morrissey and A. Pierce, Phys. Rev. D 86 (2012) 013009 [arXiv:1203.2924 [hep-ph]];

M. Carena, G. Nardini, M. Quiros and C. E. M. Wagner, arXiv:1207.6330 [hep-ph].

[43] S. Ferrara and E. Remiddi, Phys. Lett. B 53 (1974) 347.

[44] M. Hirsch, F. Staub and A. Vicente, Phys. Rev. D 85 (2012) 113013 arXiv:1202.1825 [hep-ph]].

[45] A. Abada, D. Das, A. Vicente and C. Weiland, JHEP 1209 (2012) 015 [arXiv:1206.6497 [hep-ph]].

[46] G. Aad et al. [ATLAS Collaboration], arXiv:1206.1760. 
S. Chatrchyan et al [CMS Collaboration], arXiv:1207.1898.

[47] J. Bernabéu, A. Santamaria, J. Vidal, A. Mendez and J. W. F. Valle, Phys. Lett. B 187 (1987) 303;

J. G. Körner, A. Pilaftsis and K. Schilcher, Phys. Lett. B 300 (1993) 381;

J. Bernabéu, J. G. Körner, A. Pilaftsis and K. Schilcher, Phys. Rev. Lett. 71 (1993) 2695.

[48] F. Deppisch and J. W. F. Valle, Phys. Rev. D 72 (2005) 036001.

[49] J. Kersten and A. Y. Smirnov, Phys. Rev. D 76 (2007) 073005 arXiv:0705.3221 [hep-ph]].

[50] A. Pilaftsis, Phys. Rev. D 78 (2008) 013008 arXiv:0805.1677 [hep-ph]].

[51] T. P. Cheng and L. F. Li, Phys. Rev. Lett. 45 (1980) 1908.

[52] E. Arganda and M. Herrero, Phys. Rev. D 73 (2006) 055003.

[53] H. C. Chiang, E. Oset, T. S. Kosmas, A. Faessler and J. D. Vergados, Nucl. Phys. A 559 (1993) 526.

[54] R. Kitano, M. Koike and Y. Okada, Phys. Rev. D 66 (2002) 096002.

[55] A. Ilakovac, B. A. Kniehl and A. Pilaftsis, Phys. Rev. D 52 (1995) 3993 hep-ph/9503456.

[56] R. Alonso, M. Dhen, M. B. Gavela and T. Hambye, arXiv:1209.2679 [hep-ph].

[57] J. Ellis and K. A. Olive, Eur. Phys. J. C 72 (2012) 2005 arXiv:1202.3262.

[58] M. Carena, H. E. Haber, S. Heinemeyer, W. Hollik, C. E. M. Wagner and G. Weiglein, Nucl. Phys. B 580 (2000) 29 [hep-ph-0001002].

[59] J. S. Lee, M. Carena, J. Ellis, A. Pilaftsis and C. E. M. Wagner, arXiv:1208.2212 [hep-ph];

J. S. Lee, M. Carena, J. Ellis, A. Pilaftsis and C. E. M. Wagner, Comput. Phys. Commun. 180 (2009) 312;

J. S. Lee, A. Pilaftsis, M. S. Carena, S. Y. Choi, M. Drees, J. R. Ellis and C. E. M. Wagner, Comput. Phys. Commun. 156 (2004) 283.

[60] S. Heinemeyer, M. J. Herrero, S. Penaranda and A. M. Rodriguez-Sanchez, JHEP 1105 (2011) 063.

[61] P. Chankowski and S. Pokorski, Int. J. Mod. Phys. A 17 (2002) 575 hep-ph/0110249.

[62] S. Petcov, S. Profumo, Y. Takanishi and C. Yaguna, Nucl. Phys. B 676 (2004) 453-480 hep-ph/0306195.

[63] K. Hayasaka et al. [Belle Collaboration], Phys. Lett. B 687 (2010) 139.

[64] B. Aubert et al. [Babar Collaboration], Phys. Rev. Lett. 104 (2010) 021802.

[65] The Mu2e Project at Fermilab: http://mu2e.fnal.gov/.

[66] Y. Mori et al. [The PRIME Working Group], "An experimental search for $\mu^{-} \rightarrow e^{-}$conversion process at the ultimate sensitivity of the order of $10^{-18}$ with PRISM", LOI-25, http://www-ps.kek.jp/jhf-np/LOIlist/LOIlist.html

[67] The X-Project: http://projectx.fnal.gov.

[68] S. Ritt et al. [MEG Collaboration], Nucl. Phys. Proc. Suppl. 162 (2006) 279.

[69] M. Drees, M. Goldbole and P. Roy, Theory and Phenomenology of Sparticles, World Slientific, 2004. 